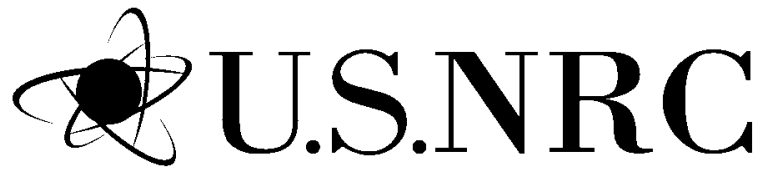

NUREG/CR-7128

ANL-11/27

United States Nuclear Regulatory Commission

Protecting People and the Environment

\title{
Void Swelling and Microstructure of Austenitic Stainless Steels Irradiated in the BOR-60 Reactor
}




\section{AVAILABILITY OF REFERENCE MATERIALS IN NRC PUBLICATIONS}

\section{NRC Reference Material}

As of November 1999, you may electronically access NUREG-series publications and other NRC records at NRC's Public Electronic Reading Room at http://www.nrc.gov/reading-rm.html. Publicly released records include, to name a few, NUREG-series publications; Federal Register notices; applicant, licensee, and vendor documents and correspondence; NRC correspondence and internal memoranda; bulletins and information notices; inspection and investigative reports; licensee event reports; and Commission papers and their attachments.

NRC publications in the NUREG series, NRC regulations, and Title 10, "Energy," in the Code of Federal Regulations may also be purchased from one of these two sources.

1. The Superintendent of Documents

U.S. Government Printing Office Mail Stop SSOP

Washington, DC 20402-0001

Internet: bookstore.gpo.gov

Telephone: 202-512-1800

Fax: 202-512-2250

2. The National Technical Information Service Springfield, VA 22161-0002

www.ntis.gov

1-800-553-6847 or, locally, 703-605-6000

A single copy of each NRC draft report for comment is available free, to the extent of supply, upon written request as follows:

Address: U.S. Nuclear Regulatory Commission

Office of Administration

Publications Branch

Washington, DC 20555-0001

E-mail: DISTRIBUTION.RESOURCE@NRC.GOV

Facsimile: 301-415-2289

Some publications in the NUREG series that are posted at NRC's Web site address

http://www.nrc.gov/reading-rm/doc-collections/nuregs are updated periodically and may differ from the last printed version. Although references to material found on a Web site bear the date the material was accessed, the material available on the date cited may subsequently be removed from the site.

\section{Non-NRC Reference Material}

Documents available from public and special technical libraries include all open literature items, such as books, journal articles, transactions, Federal Register notices, Federal and State legislation, and congressional reports. Such documents as theses, dissertations, foreign reports and translations, and non-NRC conference proceedings may be purchased from their sponsoring organization.

Copies of industry codes and standards used in a substantive manner in the NRC regulatory process are maintained at-

The NRC Technical Library

Two White Flint North

11545 Rockville Pike

Rockville, MD 20852-2738

These standards are available in the library for reference use by the public. Codes and standards are usually copyrighted and may be purchased from the originating organization or, if they are American National Standards, from-

American National Standards Institute

11 West $42^{\text {nd }}$ Street

New York, NY 10036-8002

www.ansi.org

212-642-4900

Legally binding regulatory requirements are stated only in laws; NRC regulations; licenses, including technical specifications; or orders, not in NUREGseries publications. The views expressed in contractor-prepared publications in this series are not necessarily those of the NRC.

The NUREG series comprises (1) technical and administrative reports and books prepared by the staff (NUREG-XXXX) or agency contractors (NUREG/CR-XXXX), (2) proceedings of conferences (NUREG/CP-XXXX), (3) reports resulting from international agreements (NUREG/IA-XXXX), (4) brochures (NUREG/BR$X X X X)$, and (5) compilations of legal decisions and orders of the Commission and Atomic and Safety Licensing Boards and of Directors' decisions under Section 2.206 of NRC's regulations (NUREG-0750).

DISCLAIMER: This report was prepared as an account of work sponsored by an agency of the U.S. Government. Neither the U.S. Government nor any agency thereof, nor any employee, makes any warranty, expressed or implied, or assumes any legal liability or responsibility for any third party's use, or the results of such use, of any information, apparatus, product, or process disclosed in this publication, or represents that its use by such third party would not infringe privately owned rights. 
United States Nuclear Regulatory Commission

Protecting People and the Environment

\section{Void Swelling and Microstructure of Austenitic Stainless Steels Irradiated in the BOR-60 Reactor}

Manuscript Completed: June 2011

Date Published: November 2012

Prepared by:

Y. Chen, ${ }^{1}$ Y. Yang, ${ }^{2}$ Y. Huang, ${ }^{2}$ T. Allen, ${ }^{2}$

B. Alexandreanu, ${ }^{1}$ and K. Natesan ${ }^{1}$

${ }^{1}$ Argonne National Laboratory

Argonne, IL 60439

${ }^{2}$ University of Wisconsin-Madison Madison, WI 53706

NRC Project Manager, A. S. Rao

NRC Job Code N6519

Office of Nuclear Regulatory Research 



\begin{abstract}
As nuclear power plants age and neutron fluence increases, detrimental effects resulting from radiation damage have become an increasingly important issue for the operational safety and structural integrity of core internal components. In this study, irradiated specimens of reactor core internal components were characterized by transmission electron microscopy. The specimens had been irradiated to 5.5-45 dpa in the BOR- 60 reactor at a dose rate close to $10^{-6} \mathrm{dpa} / \mathrm{s}$ and temperature of about $320^{\circ} \mathrm{C}$. No voids were observed in the austenitic stainless steels and nickel alloys at all doses. Despite the possibility that fine voids below the TEM resolution limit may be present, it was clear that void swelling was insignificant in all examined alloys up to $45 \mathrm{dpa}$. Irradiated microstructures of the studied alloys were dominated by a high density of Frank loops. The mean size and density of the Frank loops varied from one material to another, but saturated with increasing dose above $\sim 10 \mathrm{dpa}$. While no irradiation-induced precipitations were present below $24.5 \mathrm{dpa}$, fine precipitates were evident in several alloys at $45 \mathrm{dpa}$.
\end{abstract}

\title{
Paperwork Reduction Act Statement
}

This NUREG does not contain information collection requirements and, therefore, is not subject to the requirements of the Paperwork Reduction Act of 1995 (44 U.S.C. 3501 et seq.).

\section{Public Protection Notification}

The NRC may not conduct or sponsor, and a person is not required to respond to, a request for information or an information collection requirement unless the requesting document displays a current valid OMB control number. 



\section{Contents}

Abstract

Figures

vii

Tables

ix

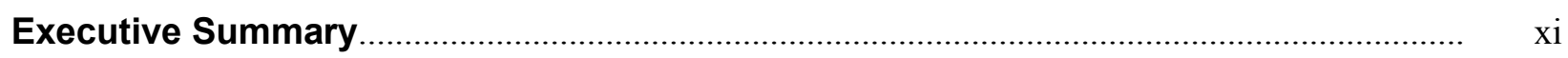

Foreword



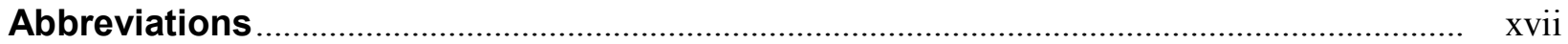

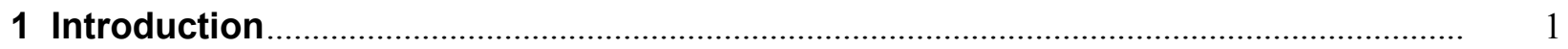

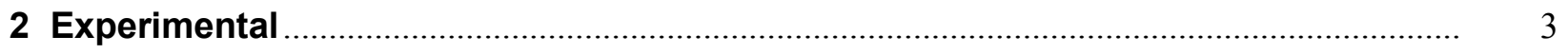

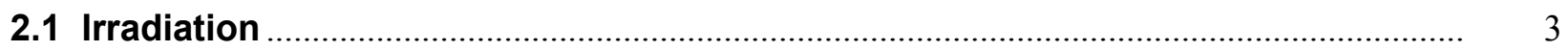

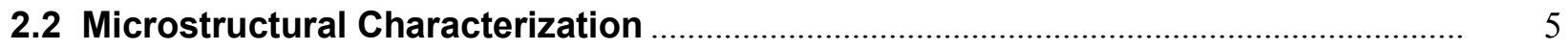

2.2.1 TEM Specimen Preparation ............................................................................... 5

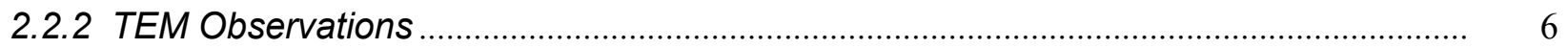



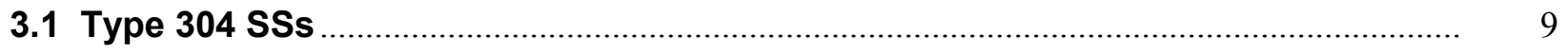

3.1.1 Solution-Annealed 304 SSs with Different Sulfur Content............................................. 9

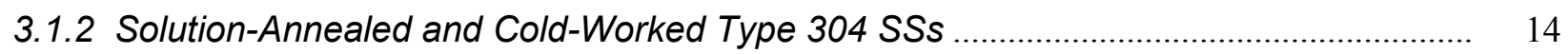

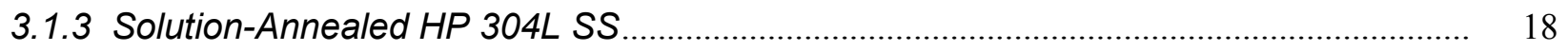



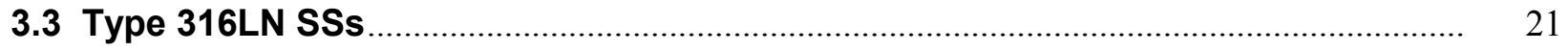

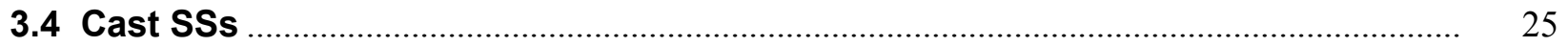

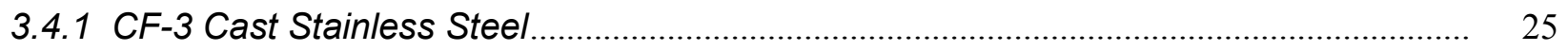

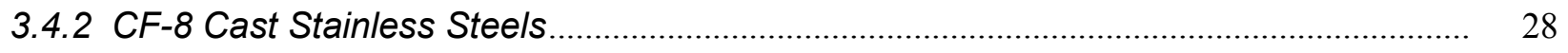

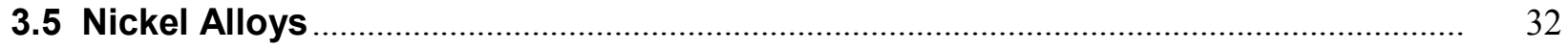




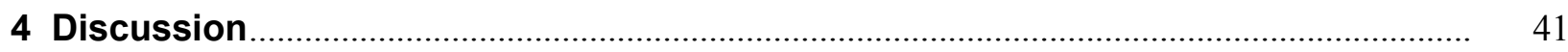

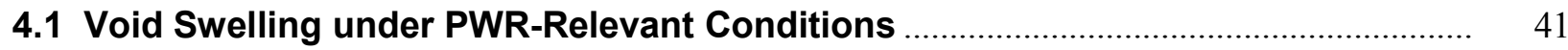

4.1.1 Void Swelling Mechanism and Key Variables ...............................................................

4.1.2 Irradiation Conditions of BOR-60 and PWR Internals ......................................................

4.2 Irradiated Microstructure at PWR-Relevant Temperature ...................................... 47

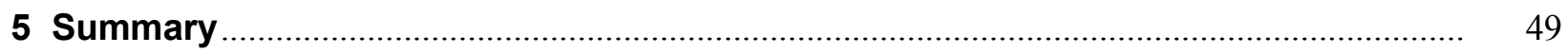






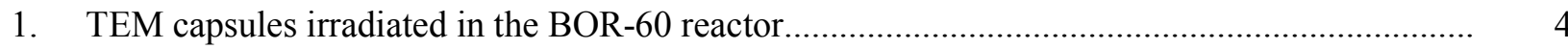

2. Modified Teflon diaphragm cap for electropolishing ....................................................... 6

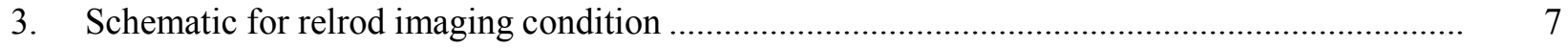

4. Nonirradiated microstructure of SA Type 304 SS (Mat. A2) with a few dislocations............... 10

5. Irradiated microstructure of SA Type 304 SS (Mat. A2) at 24.5 and 45 dpa: (a, b) BF images, (c, d) relrod DF images, and (e, f) loop size distributions.

6. Irradiated microstructure of SA Type $304 \mathrm{SS}$ with low sulfur (Mat. A3) at 10.2, 24.5, and 45.0 dpa: (a-c) BF images and (d-f) relrod DF images.

7. Microstructure of SA Type 304 SS (Mat. B1) irradiated to 45.0 dpa: (a) BF image and (b) relrod DF image.

8. Nonirradiated microstructure of CW Type 304 SS (Mat. B2): (a) dislocation networks and twins and (b) high density dislocations.

9. Irradiated microstructure of CW Type 304 SS (Mat. B2) at 24.5 dpa

10. Irradiated microstructure of SA HP 304L SS (Mat. A8) at 45 dpa: (a) BF image and (b) relrod DF image.

11. Irradiated microstructure of SA Type 347 SS (Mat. D1) at 45 dpa: (a) BF image, (b) relrod DF image, and $(c, d)$ precipitates at a grain boundary and within a grain.

12. Nonirradiated microstructure of Type 316LN SS (Mat. B3).

13. Irradiated microstructure of Type 316LN SS (Mat. B3): (a, b) 24.5 dpa and (c, d) 45.0 dpa......

14. Irradiated microstructure of Type 316LN-Ti SS (Mat. B4) at $45 \mathrm{dpa}$.

15. Nonirradiated microstructure of CF-8 cast SS (Mat. C4).

16. Irradiated microstructure of CF-3 with $14 \%$ ferrite (Mat. C1) at $24.5 \mathrm{dpa}$ : (a) general view of duplex structure, (b) relrod DF images showing Frank loops in austenitic phase, and

(c) WBDF image of irradiation defects in ferrite.

17. Irradiated microstructure of CF-3 with $14 \%$ ferrite (Mat. C1) at 45 dpa: (a) general duplex microstructure, (b) relrod DF image showing Frank loops in austenite, and (c) precipitates in austenite. 
18. Irradiated microstructure of CF-8 (Mats. C2 and C4) at 24.5 dpa: (a) general view of duplex microstructure of Mat. C2, (b) relrod DF image showing Frank loops in austenitic phase in Mat. C2, (c) irradiation defects in austenite in Mat. C4, and (d) irradiation defects in ferrite in Mat. C4.

19. Irradiated microstructure of CF-8 with $14 \%$ ferrite at 45 dpa (Mat. C2): (a) general view of duplex microstructure and (b) relrod DF image of Frank loops in austenite.

20. Fine precipitates in austenite of CF-8 at 45 dpa visualized in a DF image with ultra-reflection.

21. Nonirradiated microstructure of nickel alloys (Mat. E1 and E2): (a, b) GBE 690 and (c, d) Alloy 690.

22. Diffraction pattern and EDS spectrum of $\mathrm{M}_{23} \mathrm{C}_{6}$ precipitate in nonirradiated Alloy 690.

23. Irradiated microstructure of GBE 690 (Mat. E1) at 24.5 dpa: (a) cavities possibly left from fall-off of precipitates, (b) BF image of irradiation defects, and (c) relrod DF image of dislocation loops.

24. Irradiated microstructure of Alloy 690 (Mat. E2) at 24.5 dpa: (a) precipitates and cavities, (b) BF image of irradiation defects, and (c) relrod DF image of dislocation loops.

25. Irradiated microstructure of GBE 690 (Mat. E1) at 45 dpa.

26. Void swelling measured on $12 \%$ CW 316 SSs irradiated in EBR-II.

27. Schematic of temperature and dose dependence of void swelling.

28. Relation of peak swelling temperature and dose rate.

29. Effect of cold-work on void swelling.

30. Density and size of Frank loops in irradiated 304 SA with high S content. 


\section{Tables}



2. Specimens irradiated in the Boris- 6 and -7 experiments.................................................... 4

3. Displacement damage doses calculated by RIAR .............................................................. 5

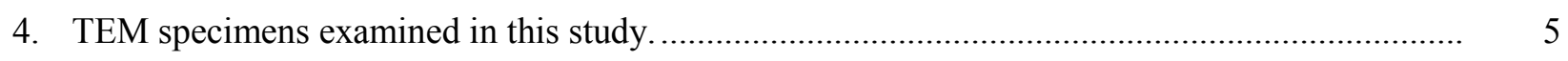

5. Observations and measurements of SA Type 304 SS with high sulfur content (Mat. A2)........... 10

6. Observations and measurements of SA Type 304 SS with low sulfur content (Mat. A3)............ 12

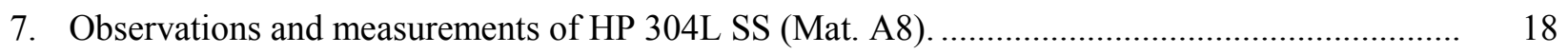

8. Observations and measurements of SA Type 347 SS (Mat. D1)............................................... 19

9. Observations and measurements of SA Type 316LN SS (Mat. B3) ............................................ 21

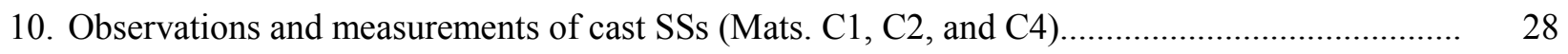

11. Observations and measurements of Alloy 690s (Mats. E1 and E2) .........................................

12. Summary of TEM observations on the irradiated BOR-60 specimens. ....................................... 



\section{Executive Summary}

Reactor core internal components are subjected to neutron irradiation in light water reactors (LWRs). Under fast neutron bombardments, a large number of atoms are displaced from their lattice sites, forming point defects and defect clusters. The evolution of these defects at irradiation temperatures gives rise to various irradiation effects. Void swelling is such an irradiation effect that can potentially have a significant impact on the service performance of core internals. Because of the low operation temperatures for boiling water reactors (BWRs) and pressurized water reactors (PWRs), void swelling was initially not considered a key problem. These predictions are based on the fast reactor data, which may not properly represent the irradiation conditions in LWRs. A lower damage rate in LWRs may reduce the incubation dose limit and shift the peak swelling temperatures toward lower values. Combined with concerns that temperatures up to $450^{\circ} \mathrm{C}$ might exist in the thick section of components due to $\gamma$-heating, void swelling could become a critical issue for PWRs at the end-of-life dose level. In addition to void swelling, other irradiation defects and precipitations are also of concern for aging reactor internals. Along with voids or cavities, irradiation-induced dislocation loops, defect clusters, and precipitates have significant impacts on the mechanical properties and service performance of austenitic stainless steels (SSs). As life extension of many aged power plants is under consideration, a better understanding of the microstructural evolution and accurate assessments of irradiation defects in internal components are essential for the safe and economic operation of LWRs.

In the present study, the microstructures of irradiated SSs and nickel-base alloys were characterized by transmission electron microscopes (TEMs). Neutron irradiations were carried out in the BOR-60 reactor, a sodium-cooled fast breeder reactor in Russia. Three-millimeter TEM disks of various austenitic SSs and two heats of Alloy 690 were irradiated to four doses $(5.5,10.2,24.5$ and $45 \mathrm{dpa})$ at $\sim 320^{\circ} \mathrm{C}$ in two irradiation experiments. The dose rate was approximately $10^{-6} \mathrm{dpa} / \mathrm{s}$, higher than the typical dose rates experienced by PWR internals $\left(\sim 10^{-7} \mathrm{dpa} / \mathrm{s}\right)$.

A total of 26 specimens from 13 materials were selected for TEM examination. The thickness of the TEM samples varied from 250 to $350 \mu \mathrm{m}$. Following irradiation, the TEM foils were prepared by electropolishing and were examined with a JEOL-100CX or JEOL-200CX TEM. Some nonirradiated control specimens were also examined with a Philips CM200 TEM. Dislocation loops were analyzed using relrod dark-field images, and voids or cavities were examined by the through-focus technique under high magnification.

For all specimens, the irradiated microstructures were dominated by a high density of Frank loops, which varied in mean size and density for different alloys. The dislocation loop structure seemed to saturate above $10 \mathrm{dpa}$, and little difference was observed beyond saturation. While no irradiation-induced precipitates were present below $24.5 \mathrm{dpa}$, fine precipitates were evident in some 45 -dpa specimens. The exact nature of these precipitates could not be determined due to the TEM resolution limit.

No voids were observed in any of the specimens imaged with the through-focus technique under high magnification. Despite the possibility that fine voids below the TEM resolution limit may be present, it is clear that void swelling was insignificant in all examined alloys up to $45 \mathrm{dpa}$. The absence of voids, even at the highest dose, suggests that the irradiation conditions in this study may be inadequate to foster steady-state void growth, and void swelling was still within an incubation period. While the irradiation temperature $\left(\sim 320^{\circ} \mathrm{C}\right)$ is prototypical for PWR core internals, the displacement damage rate of the BOR-60 irradiation is higher than that for PWR core internals. A shift in the peak void swelling temperature may have resulted from the high dose rate used in this study. In addition, a low population of thermal neutrons in the BOR-60 spectrum, which leads to a lower production rate of transmutation helium, may have contributed to the absence of void swelling. 



\section{Foreword}

In the early $1980 \mathrm{~s}$, research on irradiation degradation in liquid metal, fast breeder reactors (LMFBRs) produced an enormous quantity of data describing the kinetics and consequences of void swelling in stainless steels (SSs) and nickel-based alloys proposed for use in those reactors. However, breeder reactors have temperatures and neutron flux rates that are considerably higher than those that are characteristic of light-water reactors (LWRs). These differences make it difficult to simply extrapolate LMFBR data to LWR operational regimes. In 1995, NRC joined an international collaborative program called the cooperative irradiation assisted stress corrosion cracking research (CIR) program that broadened the range of operating parameters to include LWR conditions. The staff also participated in the follow-up programs: CIR II (2000) and CIR II Extension (2005) that targeted high fluences and, thus, once again utilized irradiations in fast breeder reactors such as BOR-60. The CIR programs contributed to the fundamental understanding of the causes for irradiation-assisted stress corrosion cracking and the microstructural changes in irradiated authentic SS reactor core internals.

In response to a 2009 request from the Office of Nuclear Reactor Regulation, the Office of Nuclear Regulatory Research evaluated void swelling in LWR reactor core internals during reactor operation beyond 40 years. The staff initially evaluated results obtained from the CIR II Extension program. As mentioned above, inconsistencies in irradiation parameters between LWRs and BOR-60 yielded few results that were directly applicable to LWRs. However, the examination of BOR-60 samples provides significant generic information on the mechanisms of void nucleation and growth as a function of neutron fluence, dose rate, and temperature for a wide range of materials.

The present study is the beginning of systematic void swelling work, and this report was produced to identify the onset of void nucleation, which was determined solely from the examination of the irradiated specimen microstructure under transmission electron microscope. Austenitic SSs and nickel-base alloy specimens were irradiated in the BOR-60 reactor to doses up to 45 dpa at temperatures of approximately $320^{\circ} \mathrm{C}$. No void swelling was identified in these samples. However, these results are not necessarily prototypical because the dose rates in the BOR-60 reactor are significantly higher than in LWRs. At high dose rates, the defects cannot readily coalesce to form voids. Therefore the present study expands the state-of-knowledge but the findings are not definitive because the LWR thermal spectra are more conducive to the nucleation of voids.

Follow-on research will explore the threshold neutron irradiation dose required for nucleating the defect structure and the progression of void swelling under more representative LWR fluxes, temperatures and neutron energy spectra. The investigation will encompass the evolution of various defect structures into voids, and will evaluate the effect of void swelling on the crack growth rate and fracture toughness of SS internal materials. Once complete, these research findings will support the staff's evaluation of aging management programs in subsequent license renewal period. The results may also be used to determine appropriate inspection and flaw disposition procedures for reactor internals, and in ASME code development for conditions within Title 10, Section 50.55a, "Codes and Standards," of the Code of Federal Regulations (10CFR50.55a). 



\section{Acknowledgments}

The authors would like to thank Drs. O. K. Chopra, H. M. Chung and W. J. Shack for their invaluable contribution to this project. The authors would like to thank Dr. Regis P. Shogan of Westinghouse, Mr. Jenssen Anders of Studsvik Nuclear AB, and Ms. Miroslava Ernestova of Nuclear Research Institute Rez for transferring the irradiated specimens from Russia to Argonne National Laboratory. L. Knoblich, E. Gruber, R. Clark, and E. Listwan, and R. Agasie are acknowledged for their contributions to the experimental effort. We are also grateful to Drs. W. H. Cullen, Jr., R. Tregoning and S. Crane for many helpful discussions and suggestions. This work is sponsored by the Office of Nuclear Regulatory Research, U.S. Nuclear Regulatory Commission, under Job Code N6519; Program Manager: A. S. Rao. 



\section{Abbreviations}

\begin{tabular}{ll} 
AISI & $\begin{array}{l}\text { American Iron and Steel Institute } \\
\text { bcc }\end{array}$ \\
BF & Bright Field \\
BWR & Boiling Water Reactor \\
CW & Cold Worked \\
DF & Dark Field \\
dpa & displacement per atom \\
EBR-II & Experimental Breeder Reactor-II \\
GBE & Grain Boundary Engineered \\
HP & High Purity \\
IASCC & Irradiation-Assisted Stress Corrosion Cracking \\
LWR & Light Water Reactor \\
PWR & Pressurized Water Reactor \\
RIAR & Research Institute of Atomic Reactors \\
SA & Solution Annealed \\
SS & Stainless Steel \\
TEM & Transmission Electron Microscopy \\
WBDF & Weak Beam Dark Field \\
WW & Warm Worked \\
\hline
\end{tabular}



Reactor core internal components are subjected to neutron irradiation in light water reactors (LWRs). Under bombardment of energetic neutrons or other fission particles, the microstructure of crystalline materials undergoes significant changes. Along the path of an intruding particle, kinetic energy dissipates in the host lattice by electron excitations, elastic collisions, and inelastic interactions. ${ }^{1}$ If the energy of elastic collisions is sufficiently high, lattice atoms are displaced from their original sites, generating vacancy and self-interstitial pairs in the crystalline material. With neutron irradiation in LWR environments, the energy of recoil atoms often exceeds the displacement threshold by many orders of magnitude, and thus a large number of neighboring atoms are displaced, forming displacement cascades. ${ }^{1-3}$ While most point defects annihilate quickly during the cooling phase of the cascades, surviving point defects evolve into irradiation defects, giving rise to various irradiation effects in reactor materials. As nuclear power plants age and neutron fluence increases, detrimental effects resulting from radiation damage have become an increasingly important issue for the operational safety and structural integrity of core internal components. Because of this crucial role, radiation damage and irradiation effects are a critical issue for safety regulation and aging management of LWRs.

Void swelling is one of the irradiation effects that potentially have a significant impact on the service performance of core internals. ${ }^{4,5}$ Excess void swelling not only leads to dimensional instability, but also can cause severe embrittlement of internal materials at elevated temperatures. ${ }^{6,7}$ Austenitic stainless steels (SSs) used in LWR core internals are known to be prone to void swelling at certain temperatures and neutron flux ranges. ${ }^{8-11}$ Nanometer-scale cavities, which are three-dimensional aggregations of vacancies, can develop in materials exposed to fast neutron bombardments. ${ }^{12} \mathrm{~A}$ direct consequence of void formation is volumetric dilation of irradiated materials. Under select irradiation conditions, volumetric swelling of more than a few percent can readily be established in SSs with moderate neutron exposure. In a well-known example given in reference [13], volume increase of a Type 316 SS fuel cladding tube used in fast breeder reactors was visible to the unaided eye. This type of instability is obviously a significant safety concern and engineering challenge.

Void swelling has been a subject of intensive study since its first discovery by Cawthorne and Fulton 12 several decades ago. A large body of work has been built up for fast breeder reactor and fusion programs. However, irradiation temperatures and dose rates are significantly higher in these nuclear systems than in LWRs. The peak temperature of void swelling was found to be around $550-600^{\circ} \mathrm{C}$ for SSs. ${ }^{14}$ Void swelling as measured in fast reactors at temperatures relevant to LWRs was insignificant and was thus not considered a key problem for either boiling water reactor (BWRs) or pressurized water reactors (PWRs) within their service life. More recently, Garner et al. ${ }^{15,16}$ pointed out that the predicted void swelling based on the fast breeder reactor data may not properly represent the situation in LWRs. A lower damage rate in LWRs $\left(\sim 10^{-7} \mathrm{dpa} / \mathrm{s}\right.$ for LWRs vs. $>10^{-6} \mathrm{dpa} / \mathrm{s}$ for fast reactors $)$ may reduce the incubation dose limit of void swelling and lower the peak swelling temperatures. Void swelling less than $1 \%$ was indeed observed in some Russian austenitic steels irradiated at PWR-relevant dose rates and temperatures. ${ }^{17}$ Combined with concerns that temperatures up to $450^{\circ} \mathrm{C}$ might exist in a thick section of components due to $\gamma$-heating, void swelling could become a critical issue for PWRs at the end-of-life dose level. This concern triggered a literature survey of void swelling under PWR-relevant conditions. ${ }^{18}$ With very limited data from decommissioned PWR components, the study confirmed that void swelling is relatively low under PWR conditions. However, because of its potential impact on aging PWRs, ${ }^{19}$ a closer look at the void swelling issue under doses and temperatures relevant to PWRs is essential for aging management and license renewal.

In addition to void formation, other irradiation-induced defects and precipitation are of concern for aging reactor internals. Along with voids or cavities, irradiation-induced dislocation loops, defect clusters, and 
precipitates can have significant impacts on the service performance of SSs. Irradiation hardening and embrittlement, deteriorated creep resistance and fracture properties, and elevated cracking susceptibility are all critical issues for SSs exposed to fast neutron bombardment. 4,11,20 It is well accepted that the irradiated microstructure, which is a function of the irradiation conditions, is responsible for the property changes. To evaluate the service performance of reactor materials, information on the irradiation defect structure and microstructural evolution is critical. For this reason, irradiation-induced microstructure has been studied extensively, and the dose dependence of microstructure evolution has been established for many reactor materials. ${ }^{9,10}$ Most data on irradiated SSs are from research programs of fast reactors or fusion systems, whose irradiation conditions are not typical for LWRs. Since microstructural evolution is a function of irradiation temperature and dose rate, special attention must be paid when this information is used for assessing aging-related issues of LWR components. As life extension of many aged power plants is under consideration, a better understanding of microstructure evolution and accurate assessments of irradiation defects in reactor internals are essential for the safe and economic operation of LWRs.

In the present work, the irradiation microstructures of austenitic stainless steels and nickel-base alloys were characterized with transmission electron microscopes (TEMs). The specimens were irradiated in the BOR-60 reactor, a fast breeder reactor, up to $\sim 45 \mathrm{dpa}$ at $\sim 320^{\circ} \mathrm{C}$. The dose rate of the BOR-60 irradiation was approximately $10^{-6} \mathrm{dpa} / \mathrm{s}$, higher than typical dose rates experienced by PWR internal components. Despite this difference, the BOR-60 specimens provide a valuable opportunity to examine a wide range of materials under laboratory-controlled irradiation conditions. The displacement damage (measured in dpa) is the most fundamental parameter that characterizes the extent of radiation damage at lattice level. The damage doses of the BOR-60 specimens, ranging from $\sim 5$ to $\sim 45 \mathrm{dpa}$, are representative for the damage levels of PWR internals after 40-60 years of life. As a laboratory-controlled experiment, neutron fluence can be monitored closely with in-situ dosimetry over the whole energy spectra of the BOR-60 irradiation. By minimizing the uncertainties in calculating neutron fluence, the final damage doses of the BOR-60 specimens can be determined rather accurately. These well-defined dose levels are crucial for studying the dose-dependent effects of radiation damage. For this reason, void swelling and irradiated microstructure were analyzed in this study for the BOR-60 specimens as a function of dose. 


\section{Experimental}

\subsection{Irradiation}

Neutron irradiations were carried out in the BOR-60 reactor, a sodium-cooled fast breeder reactor located in the Research Institute of Atomic Reactors (RIAR), Dimitrovgrad, Russia. The irradiated materials consisted of 3-mm-dia TEM disks of various austenitic SSs, four cast SSs and two Alloy 690s. Table 1 provides the chemical compositions of these materials. The TEM disks were loaded in four perforated capsules and one helium-tight capsule, as shown in Fig. 1. The dpa values shown in the figure are targeted irradiation doses. While one of the 10-dpa capsules (HE-10) was irradiated in a helium environment, all the other capsules were in direct contact with the sodium coolant during irradiation. Irradiation was performed in two experiments, Boris- 6 and -7 , and the final achieved doses were summarized in Table 3.

Table 1. Materials irradiated in BOR-60 reactor (wt.\%).

\begin{tabular}{|c|c|c|c|c|c|c|c|c|c|c|c|}
\hline \multirow{2}{*}{$\begin{array}{c}\text { Material } \\
\text { Type* }\end{array}$} & \multirow{2}{*}{$\begin{array}{c}\text { Heat } \\
\text { ID }\end{array}$} & \multirow{2}{*}{$\begin{array}{l}\text { Mat. } \\
\text { Code }\end{array}$} & \multicolumn{9}{|c|}{ Composition (wt.\%) } \\
\hline & & & $\mathrm{Ni}$ & $\mathrm{Si}$ & $\mathrm{P}$ & $\mathrm{S}$ & $\mathrm{Mn}$ & $\mathrm{C}$ & $\mathrm{N}$ & $\mathrm{Cr}$ & Other Elements \\
\hline $347 \mathrm{SA}$ & 316642 & D1 & 10.81 & 0.29 & 0.023 & 0.014 & 1.56 & 0.030 & 0.021 & 18.06 & $\mathrm{Nb} 0.60, \mathrm{Mo} 0.29, \mathrm{Cu} 0.09$ \\
\hline $347 \mathrm{CW}$ & $316642 \mathrm{CW}$ & D2 & 10.81 & 0.29 & 0.023 & 0.014 & 1.56 & 0.030 & 0.021 & 18.06 & $\mathrm{Nb} 0.60, \mathrm{Mo} 0.29, \mathrm{Cu} 0.09$ \\
\hline $304 \mathrm{SA}$ & 2333 & $\mathrm{~B} 1$ & 8.5 & 0.65 & 0.031 & 0.029 & 1.38 & 0.035 & 0.068 & 18.30 & Mo 0.37 \\
\hline $304 \mathrm{CW}$ & $2333 \mathrm{CW}$ & B2 & 8.5 & 0.65 & 0.031 & 0.029 & 1.38 & 0.035 & 0.068 & 18.30 & Mo 0.37 \\
\hline 316 LN SA & 623 & B3 & 12.20 & 0.70 & 0.007 & 0.002 & 0.97 & 0.019 & 0.103 & 17.23 & Mo 2.38, $\mathrm{Cu} 0.21$ \\
\hline 316 LN-Ti SA & 625 & $\mathrm{~B} 4$ & 12.30 & 0.72 & 0.007 & 0.002 & 0.92 & 0.012 & 0.064 & 17.25 & Mo 2.38, Ti $0.027, \mathrm{Cu} 0.21$ \\
\hline $316 \mathrm{SA}$ & $\mathrm{C} 21$ & B5 & 10.24 & 0.51 & 0.034 & 0.001 & 1.19 & 0.060 & 0.020 & 16.28 & Mo $2.08, \mathrm{~B}<0.001$ \\
\hline $316 \mathrm{CW}$ & $\mathrm{C} 21 \mathrm{CW}$ & B6 & 10.24 & 0.51 & 0.034 & 0.001 & 1.19 & 0.060 & 0.020 & 16.28 & Mo $2.08, \mathrm{~B}<0.001$ \\
\hline $316 \mathrm{WW}$ & $\mathrm{C} 21 \mathrm{WW}$ & B7 & 10.24 & 0.51 & 0.034 & 0.001 & 1.19 & 0.060 & 0.020 & 16.28 & Mo $2.08, \mathrm{~B}<0.001$ \\
\hline CF- 3 cast SS, $\delta 13.5 \%$ & 52 & $\mathrm{C} 1$ & 9.40 & 0.92 & 0.012 & 0.005 & 0.57 & 0.009 & 0.052 & 19.49 & Mo $0.35, \delta 13.5 \%$ \\
\hline CF- 8 cast SS, $\delta 13.5 \%$ & 59 & $\mathrm{C} 2$ & 9.34 & 1.08 & 0.008 & 0.007 & 0.60 & 0.062 & 0.045 & 20.33 & Mo $0.32, \delta 13.5 \%$ \\
\hline CF- 3 cast SS, $\delta 23.6 \%$ & 69 & $\mathrm{C} 3$ & 8.59 & 1.13 & 0.015 & 0.005 & 0.63 & 0.023 & 0.028 & 20.18 & Mo $0.34, \delta 23.6 \%$ \\
\hline CF- 8 cast SS, $\delta 23.4 \%$ & 68 & $\mathrm{C} 4$ & 8.08 & 1.07 & 0.021 & 0.014 & 0.64 & 0.063 & 0.062 & 20.64 & Mo $0.31, \delta 23.4 \%$ \\
\hline $304 \mathrm{SA}$, low S & $\mathrm{C} 1$ & A1 & 8.12 & 0.50 & 0.038 & 0.002 & 1.00 & 0.060 & 0.060 & 18.11 & B 0.001 \\
\hline 304 SA, high S & C9 & $\mathrm{A} 2$ & 8.75 & 0.39 & 0.013 & 0.013 & 1.72 & 0.062 & 0.065 & 18.48 & $\mathrm{~B}<0.001$ \\
\hline $304 \mathrm{SA}$, low S & $\mathrm{C} 12$ & A3 & 8.23 & 0.47 & 0.018 & 0.002 & 1.00 & 0.060 & 0.070 & 18.43 & $\mathrm{~B}<0.001$ \\
\hline $304 \mathrm{CW}$, low S & $\mathrm{C} 1 \mathrm{CW}$ & A4 & 8.12 & 0.50 & 0.038 & 0.002 & 1.00 & 0.060 & 0.060 & 18.11 & B 0.001 \\
\hline $304 \mathrm{CW}$, low S & $\mathrm{C} 12 \mathrm{CW}$ & $\mathrm{A} 5$ & 8.23 & 0.47 & 0.018 & 0.002 & 1.00 & 0.060 & 0.070 & 18.43 & $\mathrm{~B}<0.001$ \\
\hline $304 \mathrm{GBE}$ & $304 \mathrm{GBE}$ & A6 & 8.43 & 0.46 & 0.014 & 0.003 & 1.54 & 0.065 & 0.088 & 18.38 & Mo 0.51 , Co 0.22 \\
\hline $316 \mathrm{GBE}$ & $316 \mathrm{GBE}$ & $\mathrm{B} 8$ & 11.12 & 0.57 & 0.011 & 0.022 & 1.85 & 0.070 & 0.056 & 16.57 & Mo 2.27 , Co 0.10 \\
\hline $690 \mathrm{GBE}$ & $690 \mathrm{GBE}$ & E1 & 59.40 & 0.30 & - & 0.003 & 0.42 & 0.010 & - & 29.10 & Fe 10.26 \\
\hline 304 BASE & 304 BASE & A7 & 8.46 & 0.41 & 0.013 & 0.014 & 1.56 & 0.065 & 0.086 & 18.32 & Mo 0.36 , Co 0.12 \\
\hline 316 BASE & 316 BASE & B9 & 10.30 & 0.43 & 0.013 & 0.020 & 1.53 & 0.055 & 0.054 & 16.42 & Mo 2.19 , Co 0.10 \\
\hline 690 BASE & 690 BASE & E2 & 61.49 & 0.05 & - & $<0.01$ & 0.15 & 0.030 & - & 29.24 & Fe 9.02 \\
\hline HP 304L SA, high O & 945 & A8 & 9.03 & 0.03 & $<0.005$ & 0.005 & 1.11 & 0.005 & 0.003 & 19.21 & $\mathrm{O} 0.047, \mathrm{Mo}<0.005$ \\
\hline HP 304L SA, low O & 1327 & A9 & 9.54 & 0.01 & 0.001 & 0.002 & 1.12 & 0.006 & $<0.001$ & 19.71 & O 0.008, Mo 0.02 \\
\hline 304L CW & $\mathrm{C} 3 \mathrm{CW}$ & A11 & 8.91 & 0.46 & 0.019 & 0.004 & 1.81 & 0.016 & 0.083 & 18.55 & $\mathrm{~B}<0.001$ \\
\hline 304-like alloy & L5 & A12 & 9.66 & 0.90 & 0.113 & 0.028 & 0.47 & 0.006 & 0.033 & 21.00 & $\mathrm{~B}<0.001$ \\
\hline
\end{tabular}

* $\mathrm{SA}=$ solution annealed, $\mathrm{CW}=$ cold worked, $\mathrm{WW}=$ warm worked at $400^{\circ} \mathrm{C}, \mathrm{SS}=$ stainless steel, $\mathrm{GBE}=$ grain boundary engineered, $\mathrm{BASE}=$ base heat for GBE modification, $\mathrm{HP}=$ high purity, and $\delta=$ ferrite content.

The Boris-6 experiment included eight irradiation sub-cycles and several maintenance shut-downs. The Boris-7 experiment included six irradiation sub-cycles. Both irradiation experiments were conducted in the fifth row of the BOR-60 reactor core. Neutron fluence was monitored by five dosimeters located in the central channel of the irradiation rig and in baskets with the specimens. The analyses of dosimeters were carried out by RIAR staff after irradiation. ${ }^{21}$ The irradiation temperature was controlled by 
monitoring the inlet and outlet sodium temperatures, which were kept at $\approx 315$ and $325^{\circ} \mathrm{C}$, respectively. ${ }^{21}$ In addition, $\mathrm{Mg}-\mathrm{Zn}$ eutectic thermal monitors were placed among specimens in several baskets during irradiation. Post-irradiation examination of these thermal monitors indicated that the irradiation temperature of the capsules was below $343^{\circ} \mathrm{C}$ at all times.

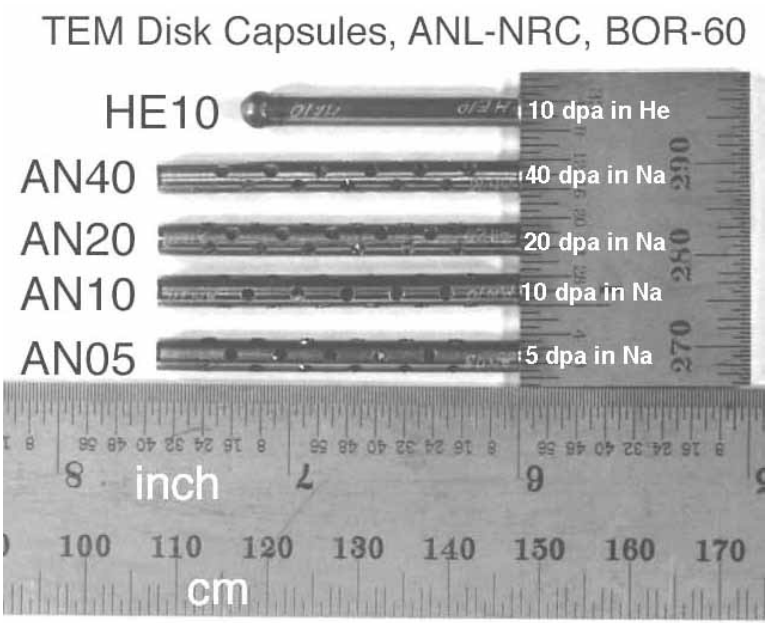

Figure 1. TEM capsules irradiated in the BOR-60 reactor (the dpa numbers in the figure are targeted irradiation doses).

The Boris- 6 and -7 irradiation specimens were discharged between 2001 and 2004 according to their target doses. All specimens were first cleaned of residual sodium and then transferred to Argonne via Westinghouse or Studsvik. All irradiated TEM disks are listed in Table 2 along with the number of specimens in the five capsules. The actual displacement damage doses received by each irradiation capsule are given in Table 3.

Table 2. Specimens irradiated in the Boris- 6 and -7 experiments.

\begin{tabular}{cccccccc}
\hline & & & \multicolumn{3}{c}{ Irradiation Capsules (Number of Specimens) } \\
\cline { 3 - 7 } Material Type & Heat ID & Mat. Code & AN 05 & AN 10 & HE 10 & AN 20 & AN 40 \\
\hline 347 SA & 316642 & D1 & 2 & 1 & 1 & 1 & 2 \\
347 CW & 316642 CW & D2 & 2 & 1 & 1 & 2 & 2 \\
ABB 304 SA & 2333 & B1 & 2 & 1 & 1 & 2 & 2 \\
ABB 304 CW & 2333 CW & B2 & 2 & 1 & 1 & 2 & 2 \\
316LN SA & 623 & B3 & 2 & 1 & 1 & 2 & 2 \\
316LN-Ti SA & 625 & B4 & 2 & 1 & 1 & 2 & 2 \\
316 SA & C21 & B5 & 2 & 1 & 1 & 2 & 2 \\
316 CW & C21 CW & B6 & 2 & 1 & 1 & 1 & 2 \\
316 WW & C21 WW & B7 & 2 & 2 & - & 2 & 2 \\
CF-3 cast & 52 & C1 & 2 & 1 & 1 & 2 & 2 \\
CF-8 cast & 59 & C2 & 2 & 2 & - & 2 & 2 \\
CF-3 cast & 69 & C3 & 2 & 1 & - & 1 & 2 \\
CF-8 cast & 68 & C4 & 2 & 2 & - & 2 & 2 \\
304 SA, low S & C1 & A1 & 2 & 1 & 1 & 2 & 2 \\
304 SA, high S & C9 & A2 & 2 & 1 & 1 & 2 & 2 \\
304 SA, low S & C12 & A3 & 2 & 1 & 1 & 2 & 2 \\
304 CW, low S & C1 CW & A4 & 2 & 1 & 1 & 2 & 2 \\
304 CW, low S & C12 CW & A5 & 2 & 1 & 1 & 2 & 2 \\
304 GBE & 304 GBE & A6 & 2 & 1 & 1 & 2 & 2 \\
316 GBE & 316 GBE & B8 & 2 & 1 & 1 & 2 & 2 \\
690 GBE & 690 GBE & E1 & 2 & 1 & 1 & 2 & 2 \\
304 BASE & 304 BASE & A7 & 2 & 1 & 1 & 2 & 2 \\
316 BASE & 316 BASE & B9 & 2 & 2 & - & 2 & 2 \\
690 BASE & 690 BASE & E2 & 2 & 2 & - & 2 & 2 \\
HP 304L SA, high O & 945 & A8 & 2 & 1 & 1 & 2 & 2 \\
HP 304L SA, low O & 1327 & A9 & 2 & 2 & 2 & 2 & 2 \\
304L SA & C3 & A10 & - & - & - & - & - \\
304L CW & C3 CW & A11 & 2 & 1 & 1 & 2 & 2 \\
304-like alloy & L5 & A12 & 2 & 1 & 1 & 2 & 2 \\
\hline
\end{tabular}


Table 3. Displacement damage doses calculated by RIAR 21

\begin{tabular}{ccc}
\hline Capsule ID & Targeted Dose (dpa) & Achieved Dose $\left(\mathrm{dpa}^{*}\right)$ \\
\hline AN 05 & 5 & 5.5 \\
AN 10 & 10 & 10.2 \\
AN 20 & 20 & 24.5 \\
HE 10 & 10 & 11.8 \\
AN 40 & 40 & 45.0 \\
\hline
\end{tabular}

* Calculated with Norgett-Robinson-Torrens (NRT) model.

\subsection{Microstructural Characterization}

Microstructural characterization was performed on standard 3-mm-dia TEM specimens. A total of 26 irradiated specimens from 13 materials were selected for microstructural examination. The original thickness of the TEM samples varied from 250 to $350 \mu \mathrm{m}$. Table 4 shows the specimens and their irradiation doses included in this study. The irradiated specimens were electropolished and examined with a JEOL-100CX TEM at Argonne, a JEOL-200CX TEM or Philips CM-200 TEM at the University of Wisconsin-Madison. Most of the nonirradiated control specimens were also examined with the Philips CM200 TEM at the University of Wisconsin-Madison.

Table 4. TEM specimens examined in this study.

\begin{tabular}{llccccc}
\hline \multirow{2}{*}{ Material Type } & \multirow{2}{*}{ Heat ID } & Mat. Code & \multicolumn{5}{c}{ Dose (dpa) } \\
\cline { 5 - 7 } & & & 5.5 & 10.2 & 24.5 & 45.0 \\
\hline 304 SA, high S & C9 & A2 & $\sqrt{n}$ & $\sqrt{ }$ & $\sqrt{ }$ & $\sqrt{ }$ \\
304 SA, low S & C12 & A3 & - & $\sqrt{ }$ & $\sqrt{ }$ & $\sqrt{ }$ \\
ABB 304 SA & 2333 & B1 & - & - & - & - \\
ABB 304 CW & 2333 CW & B2 & - & - & $\sqrt{ }$ & - \\
HP 304L SA, high O & 945 & A8 & - & - & $\sqrt{ }$ & $\sqrt{ }$ \\
347 SA & 316642 & D1 & - & - & $\sqrt{ }$ & $\sqrt{ }$ \\
316LN SA & 623 & B3 & $\sqrt{ }$ & $\sqrt{ }$ & $\sqrt{ }$ \\
316LN-Ti SA & 625 & B4 & - & - & - & $\sqrt{ }$ \\
CF-3 cast & 52 & C1 & - & - & $\sqrt{ }$ & $\sqrt{ }$ \\
CF-8 cast & 59 & C2 & - & - & $\sqrt{ }$ & - \\
CF-8 cast & 68 & C4 & - & - & $\sqrt{ }$ & $\sqrt{ }$ \\
690 GBE & 690 GBE & E1 & - & - & $\sqrt{ }$ & - \\
690 BASE & 690 BASE & E2 & - & - & $\sqrt{ }$ \\
\hline
\end{tabular}

\subsubsection{TEM Specimen Preparation}

A foil of several hundred nanometer thickness is required for a TEM examination. The most common technique to obtain a TEM thin foil is electropolishing, in which a wedge-shaped thin area surrounding a perforation is created at the TEM disk center. To ensure a large thin area with a small wedge angle, the TEM disks were thinned uniformly from both sides to $100-150 \mu \mathrm{m}$ prior to electropolishing. For the nonirradiated specimens, the TEM disks were ground with sand paper to reduce their thickness. For irradiated specimens, mechanical grinding was intentionally avoided due to concerns over loose contamination. The initial thinning of the irradiated specimens was carried out by either electrochemical polishing or mechanical dimpling. For electrochemical thinning, the sample holder of the electropolisher was modified by enlarging the sample hold-down diaphragm and adding extra flow channels in the polyvinyl chloride cap of the pedestal. Using the modified sample holder, all 5.5- and 24.5-dpa samples examined in this study were thinned from both sides to $100-150 \mu \mathrm{m}$ before final perforation. Mechanical dimpling was used on the 45-dpa specimens in this study to reduce their thicknesses before electrochemical perforation. A small amount of abrasive paste was used during dimpling. Also, the abrasive paste can retain removed materials to reduce the risk of contamination. Compared to the electrochemical method, the advantage of the dimpling method is to more precisely locate the perforation. 
A single-vertical-jet electropolisher from South Bay Technology Inc. (Model 550D) was used for the final electrochemical perforation. The solution used for the SS specimens contained 5\% perchloric acid, 35\% butyl cellosolve, and $60 \%$ methanol. A polishing condition of $-20^{\circ} \mathrm{C}$ and $70 \mathrm{~mA}$ with a diaphragm in place was optimal for SS specimens. For nickel alloys, the solution was $10 \%$ perchloric acid and $90 \%$ methanol. The optimal polishing condition for the nickel specimens was $-40^{\circ} \mathrm{C}$ and $110-125 \mathrm{~mA}$ with a diaphragm. For all polishing, the height of the jet nozzle was set at $3.5 \mathrm{~mm}$ from the assembly pedestal, and the flow rate was set at scale four.

To minimize radiation exposure, a Teflon diaphragm cap was fabricated to replace the original plastic diaphragm with an O-ring clamp used in the electropolisher. Figure 2 shows the modified diaphragm cap. This modification facilitated diaphragm alignment and, therefore, reduced the exposure time for sample handling considerably.

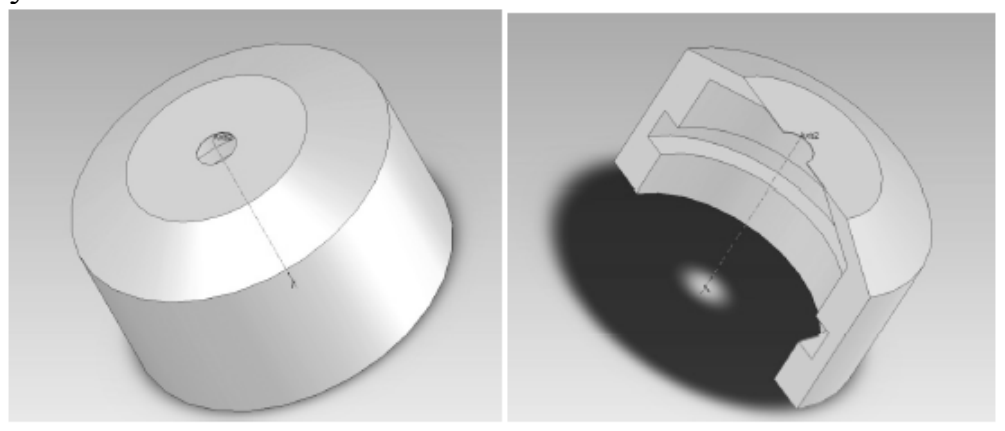

Figure 2. Modified Teflon diaphragm cap for electropolishing

Occasionally, some samples needed to be re-perforated because the area around the original perforation was inadequate. The first step for re-perforation was to coat the central area on one side (relatively flat, the first side) of the specimen with lacquer while the rim was kept free of lacquer to make electrical contact with the specimen pedestal mount. After the lacquer was dry, an aluminum foil patch was placed over the original perforation on the opposite side (the second side) of the specimen and covered with lacquer. This patch is needed to ensure that the light sensor that provides the signal to terminate electro polishing would not be activated by the original perforation. When the lacquer on the patch was dry, additional lacquer was applied to the second side of the specimen, leaving only a small uncoated area for re-perforation. The specimen was then mounted with the first side facing the jet nozzle. After re-perforation, the lacquer was dissolved from both sides in acetone and the specimen was dried in air.

\subsubsection{TEM Observations}

The irradiated SSs were examined by TEM for irradiation-induced defects, i.e., the Frank loops, voids or bubbles, and precipitation. The general irradiation microstructure was imaged under a bright-field (BF) kinematical condition, where small dark spots can be seen in irradiated specimens. This imaging condition cannot yield much detail on irradiation defects due to the complex initial microstructure of nonirradiated SSs. For a quantitative analysis, we employed relrod dark-field (DF) imaging. To examine voids and bubbles, the through-focus technique was employed to introduce a phase-contrast component (Fresnel fringes) at high magnification. Because of the resolution limit of the TEM, voids less than $1 \mathrm{~nm}$ were not readily resolved in this work.

Since Frank loops constitute a major irradiation defect in austenitic SSs, the relrod DF technique can be applied. The irradiation-induced faulted dislocation loops have a Burgers vector of $a / 3\{111\}$, and lie on $\{111\}$ planes in the austenitic structure. A relevant diffraction condition can be obtained by tilting the 
sample close to the $g=[311]$ two-beam condition near the zone axis [011]. The relrod DF images were formed by selecting the relrod streak with an objective aperture. Figure 3 shows a schematic of relrod DF imaging. With the relrod DF condition, quantitative analysis of irradiation defects is much easier because the initial nonirradiation microstructure is absent in the obtained images. One of the four variants of Frank loops can be imaged in a single relrod picture. Assuming an isotropic distribution at all orientations, the density of Frank loops can be determined. In this study, all measurements of Frank loops were performed near the perforation edge in regions that were less than 100 -nm thick to minimize the overlap of irradiation defects.



$1 / 4$ of total faulted loops

Rel-rods streaks from faulted loops on planes [111]

[011] zone axis

Figure 3. Schematic for relrod imaging condition 



\section{Results}

\subsection{Type 304 SSs}

\subsubsection{Solution-Annealed 304 SSs with Different Sulfur Content}

Two solution-annealed (SA) Type 304 SSs (Mat. Codes A2 and A3) were selected for TEM observation. Their chemical compositions are given in Table 1. While both alloys are within the American Iron and Steel Institute (AISI) specification, they differ significantly in sulfur content. Mat. A2 contains 0.013 wt.\% sulfur, compared to 0.002 wt.\% in Mat. A3. Also, Mat. A2 has greater manganese content.

\subsubsection{Type 304 SS with high S}

Figure 4 is a TEM image of nonirradiated Type 304 SS with high-sulfur content (Mat. A2). Few dislocations can be seen in the nonirradiated microstructure as a consequence of the solution-annealed treatment performed prior to neutron exposure. The nonirradiated specimen is also free of any precipitation. The irradiated microstructure, by contrast, is dominated by a high density of dislocation loops difficult to resolve under a BF condition, as shown in Figs. 5a and 5b. A similar microstructure was also seen in 5.5- and 10.2-dpa specimens despite their dose differences. No voids or cavities were observed in the irradiated specimens using the through-focus technique. Thus, no swelling is anticipated in this material under these irradiation conditions, even at $45 \mathrm{dpa}$. While no precipitation was evident in the specimens below $24.5 \mathrm{dpa}$, some very fine precipitates (small and few in number) may be present at 45 dpa. This may indicate a dose dependent precipitation behavior in this material.

Figures 5c-5d are relrod DF images of the same areas in Figs. 5a-5b. The improved contrast of irradiation-induced dislocation loops is evident in the DF images, and a quantitative analysis becomes possible. The size and number density of dislocation loops were analyzed with DigitalMicrograph from Gatan and are plotted in Figs. 5e and 5f for 24.5- and 45-dpa specimens, respectively. The 5.5- and 10.2-dpa specimens were also imaged under the same condition and analyzed. The results for all doses are summarized in Table 5. While the average loop size is almost constant for all doses, the loop density seems to increase with increasing dose. 


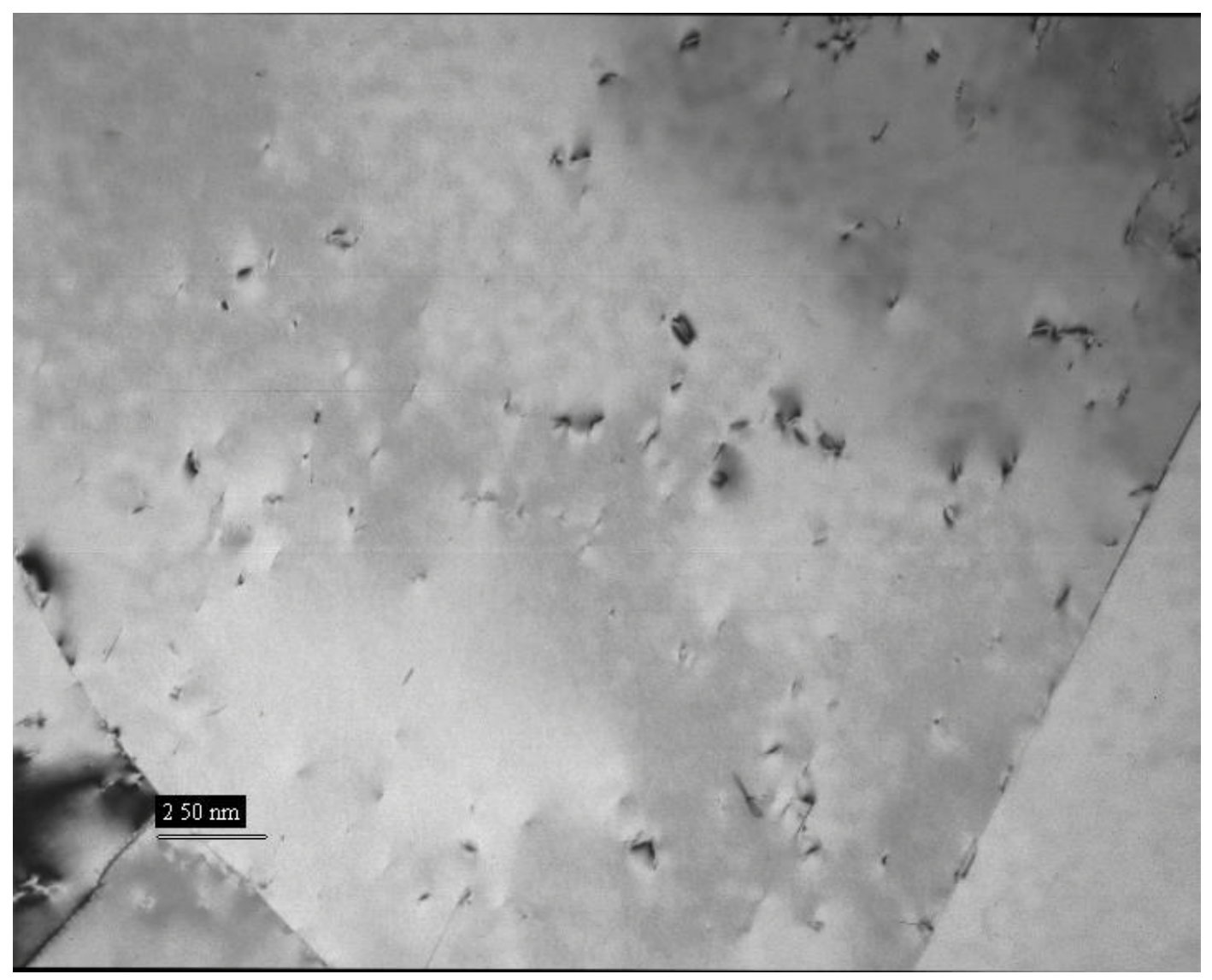

Figure 4. Nonirradiated microstructure of SA Type 304 SS (Mat. A2) with a few dislocations.

Table 5. Observations and measurements of SA Type 304 SS with high sulfur content (Mat. A2).

\begin{tabular}{ccccc}
\hline Dose (dpa) & Void & Precipitation & $\begin{array}{c}\text { Average loop size } \\
(\mathrm{nm})\end{array}$ & $\begin{array}{c}\text { Loop density } \\
\left(\times 10^{22} \mathrm{~m}^{-3}\right)\end{array}$ \\
\hline 5.5 & Not observed & Not observed & 7.9 & 3.3 \\
10.2 & Not observed & Not observed & 8.2 & 4.2 \\
24.5 & Not observed & Not observed & 8.1 & 4.6 \\
45.0 & Not observed & Possible & 7.8 & 6.7 \\
\hline
\end{tabular}






Figure 5. Irradiated microstructure of SA Type 304 SS (Mat. A2) at 24.5 and 45 dpa: (a, b) BF images, (c, d) relrod DF images, and (e, f) loop size distributions. 


\subsubsection{Type 304 SS with low S}

The irradiated microstructure of the SA Type 304 SS with low sulfur was examined at 10.2, 24.5 and 45 dpa. Similar to the high-S Type 304 SS, no voids were observed in the low-S material using the throughfocus technique that is commonly used to detect voids. Figures $6 \mathrm{a}$ to $6 \mathrm{c}$ are two-beam BF images at 10.2, 24.5 and $45 \mathrm{dpa}$. The irradiated microstructure is again dominated by fine dislocation loops, which can be seen clearly in the relrod images (Figs. $6 \mathrm{~d}$ to $6 \mathrm{f}$ ). The loop size is similar in 10.2- and 45-dpa specimens but is much larger in the 24.5-dpa specimen. The reason of the difference loop size is unknown, and may be attributed to a non-uniform initial microstructure in this material prior to irradiation. The loop density also seems to increase with irradiation dose. Table 6 summarizes the quantitative analysis of the irradiated microstructure of the low-S Type 304 SS. Except for the 24.5-dpa sample, the loop size and density of the two 304 SSs with different sulfur contents are very similar at all doses, suggesting no effects of sulfur content on the dislocation loop structure.

Table 6. Observations and measurements of SA Type 304 SS with low sulfur content (Mat. A3).

\begin{tabular}{ccccc}
\hline Dose (dpa) & Void & Precipitation & $\begin{array}{c}\text { Average loop size } \\
(\mathrm{nm})\end{array}$ & $\begin{array}{c}\text { Loop density } \\
\left(\mathrm{x} 10^{22} \mathrm{~m}^{-3}\right)\end{array}$ \\
\hline 10.2 & Not observed & Not observed & 8.1 & 2.7 \\
24.5 & Not observed & Not observed & 25.5 & 3.0 \\
45.0 & Not observed & Possible & 7.3 & 5.8 \\
\hline
\end{tabular}



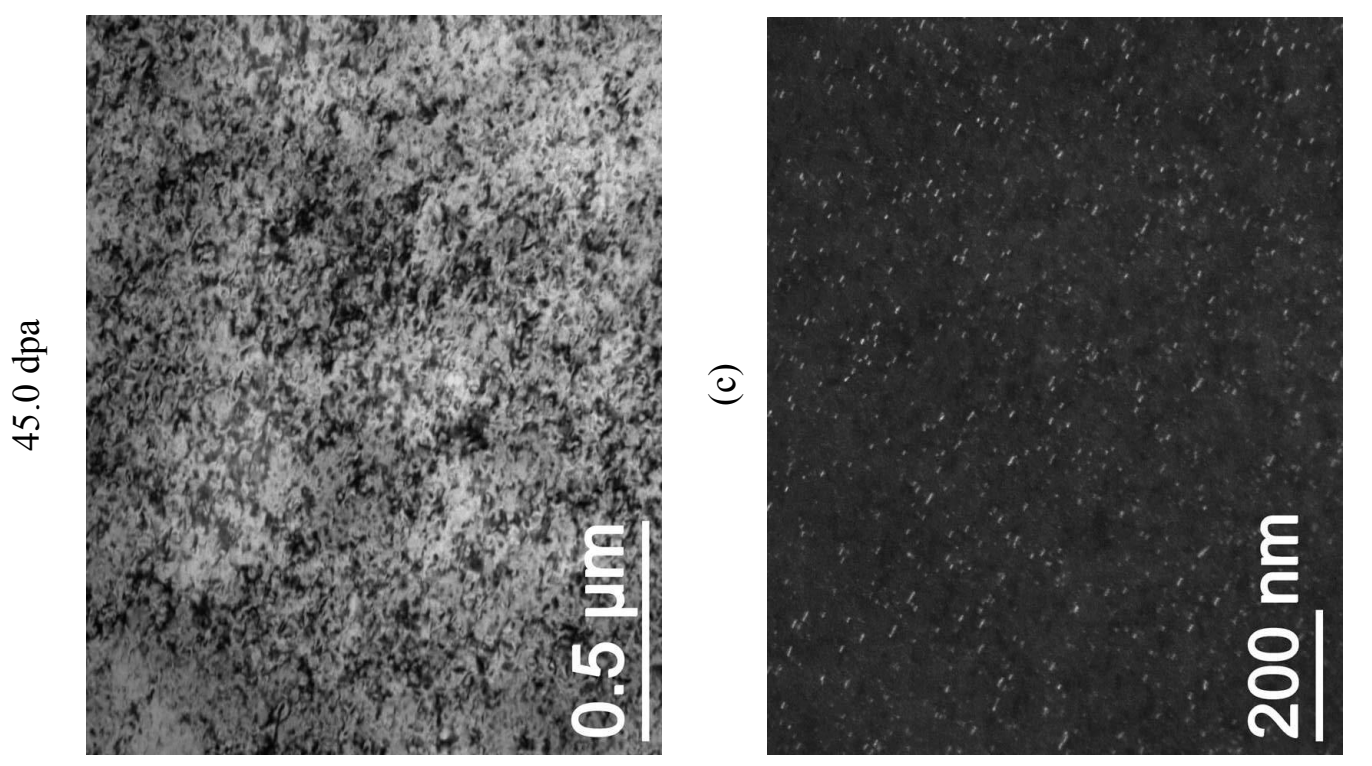

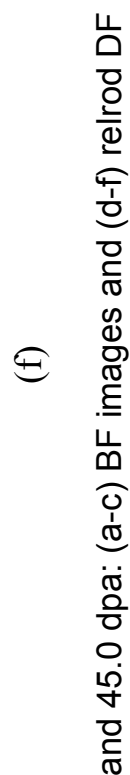
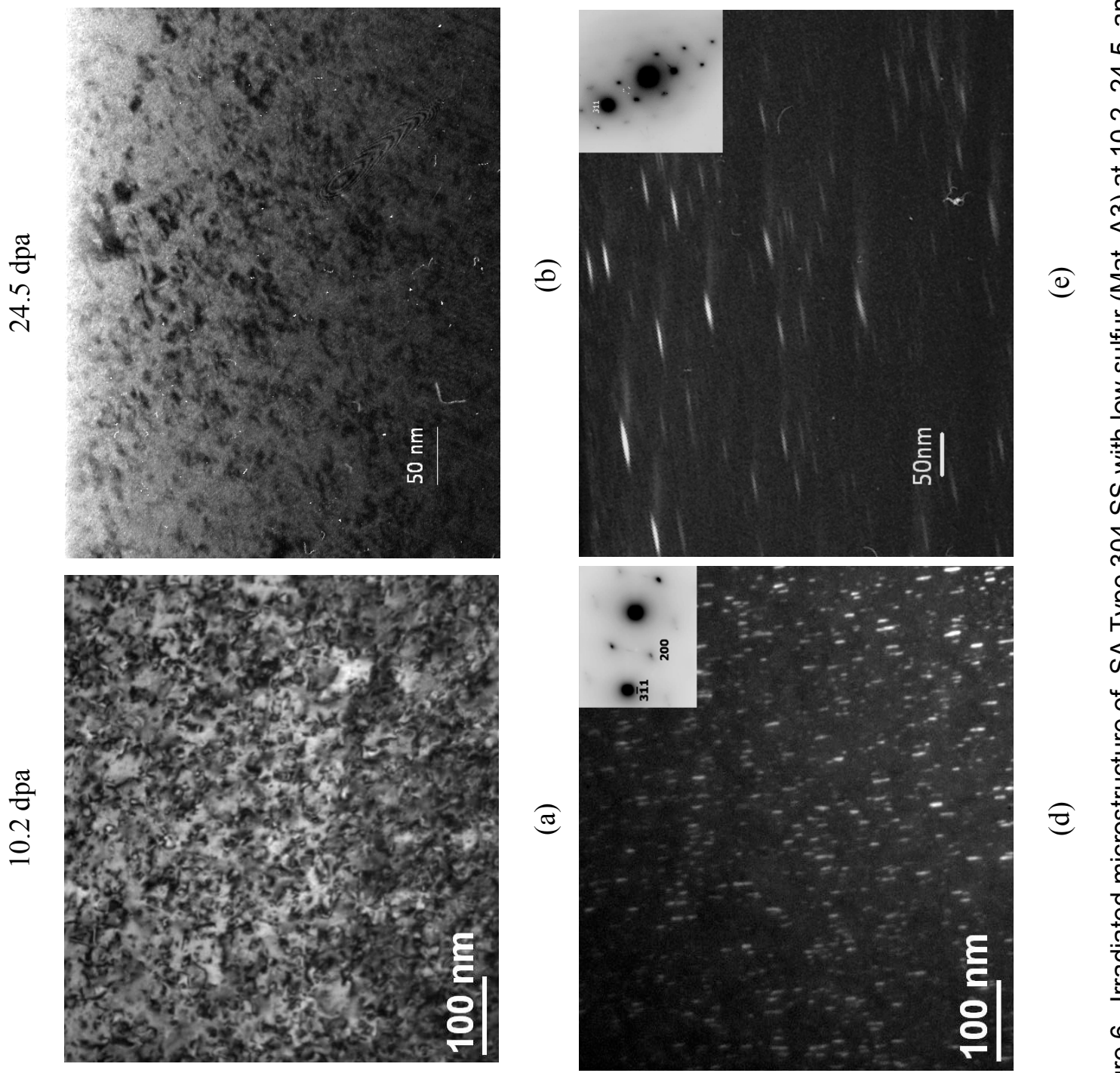

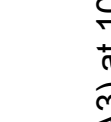

(e) $\stackrel{+\pi}{\pi}$

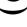

2-Beam BF images

Relrod DF images 


\subsubsection{Solution-Annealed and Cold-Worked Type 304 SSs}

Two Type 304 SSs (Mat. Codes B1 and B2) with different thermomechanical treatments were analyzed by TEM. Mat. B1 is an SA Type 304 SS with a typical chemical composition of an AISI 304 SS, as shown in Table 1. Compared to Mat. A2, this Type $304 \mathrm{SS}$ contains less carbon $(0.035 \mathrm{wt} \%)$ and much more sulfur $(0.029 \mathrm{wt} \%)$. Mat. B2 is a cold-worked $(\sim 35 \%)$ material whose composition is identical to that of Mat. B1. The irradiated SA and CW specimens were examined at 45 and $24.5 \mathrm{dpa}$, respectively.

\subsubsection{Solution-annealed Type 304 SS at 45 dpa}

The irradiated microstructure of the SA Type 304 SS is shown in Fig. 7. Small irradiation defects about $10 \mathrm{~nm}$ in size are uniformly distributed in the matrix at $45 \mathrm{dpa}$. No void or cavity is visible. Faulted dislocation loops are evident in the relrod DF images and were quantified by DigitalMicrograph. The average size and density of loops are $10.5 \mathrm{~nm}$ and $5.7 \times 10^{22} \mathrm{~m}^{-3}$, respectively, which are similar to the values obtained for Mats. A2 and A3, also SA 304 SSs, at the same dose level. The high sulfur content $(0.029 \mathrm{wt} \%)$ in this material did not have any effect on the irradiation defects.

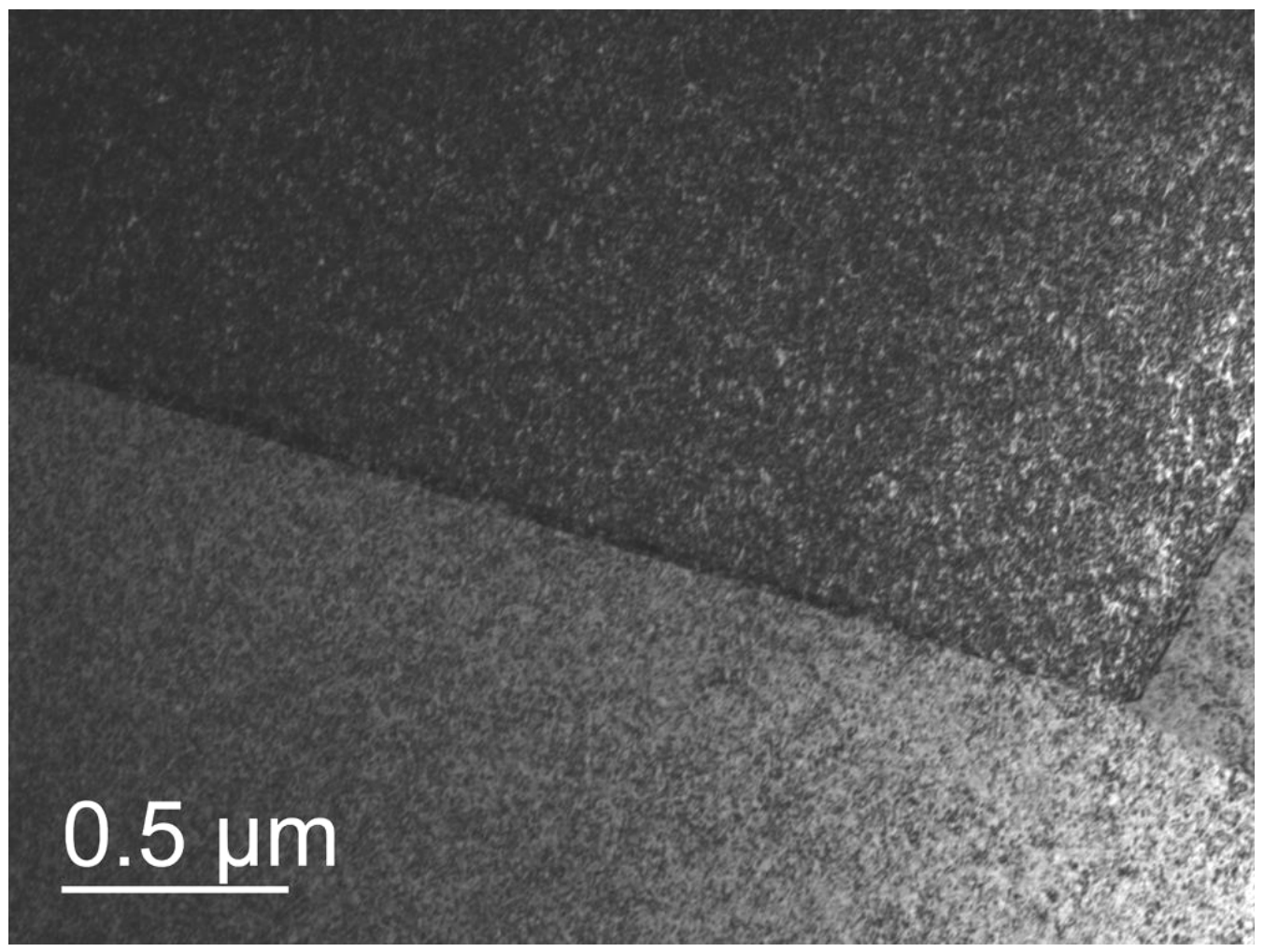

(a)

Figure 7. Microstructure of SA Type 304 SS (Mat. B1) irradiated to 45.0 dpa: (a) BF image and (b) relrod DF image. 


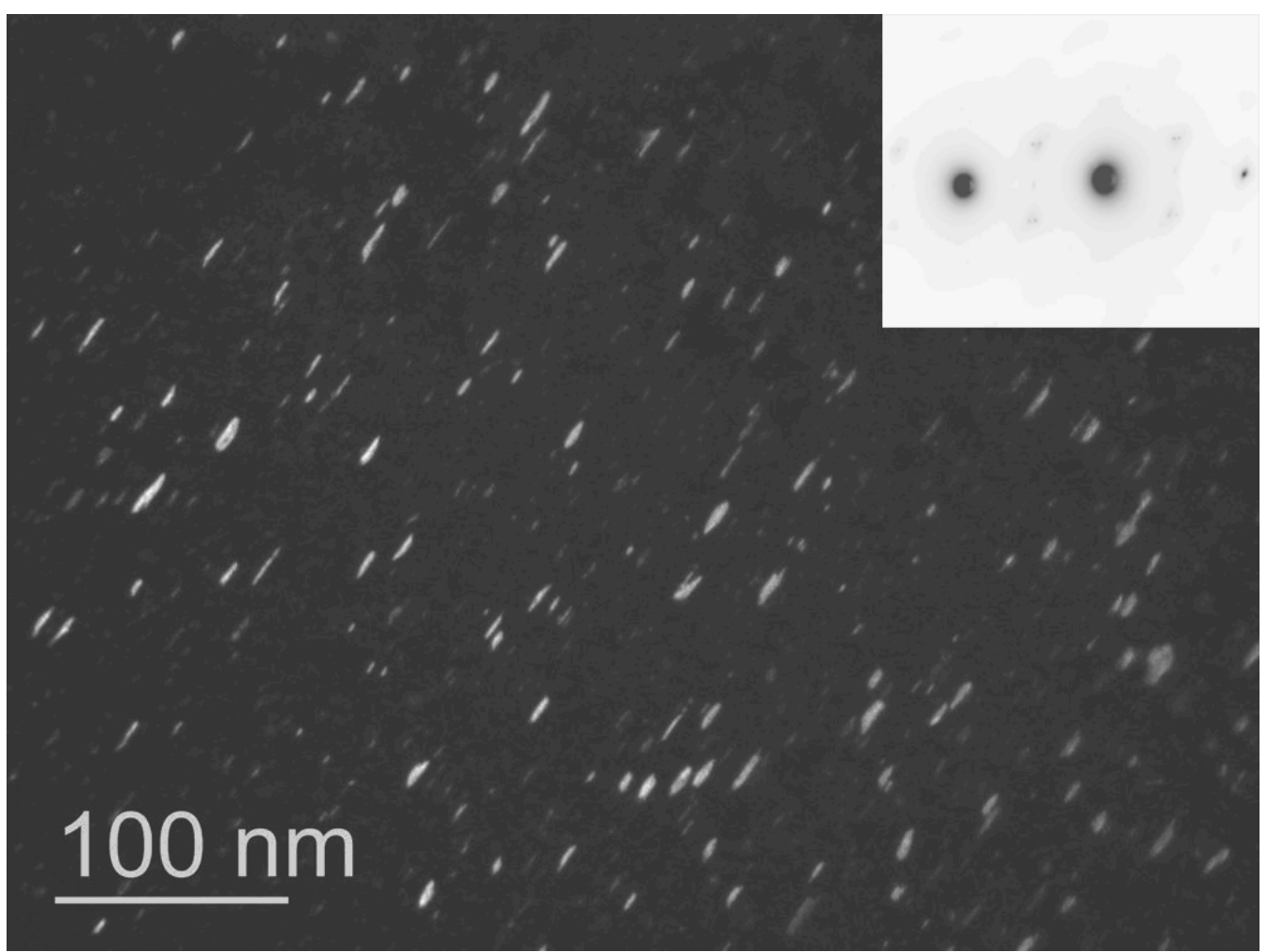

(b)

Figure 7. (Contd.)

\subsubsection{Cold-worked Type 304 SS at $24.5 \mathrm{dpa}$}

The initial microstructure of the CW Type $304 \mathrm{SS}$ is quite complex. As shown in Fig. 8, high density dislocations, cell structure, and deformation twins dominate the $\mathrm{CW}$ material. This complex microstructure makes the observation of irradiation-induced defect structure extremely difficult. As shown in Fig. 9, irradiation defects appear as "black spot damage" but cannot be resolved due to poor contrast at the kinematic condition. The distribution of irradiation defects varies from one place to another, perhaps due to interactions with the initial dislocation network. There are some large dislocation loops, which are not visible in the nonirradiated microstructure. No voids or cavities are apparent in the irradiated microstructure. Relrod DF images could not be obtained due to the heavily bent crystals in this CW material. The irradiated microstructure differ significantly between the CW (Fig. 9) and SA (Fig. 7) Type $304 \mathrm{SSs}$, and this does not appear to just be due to differences in dose. It is clear that the initial dislocation microstructure has a significant effect on microstructural evolution under neutron irradiation. 


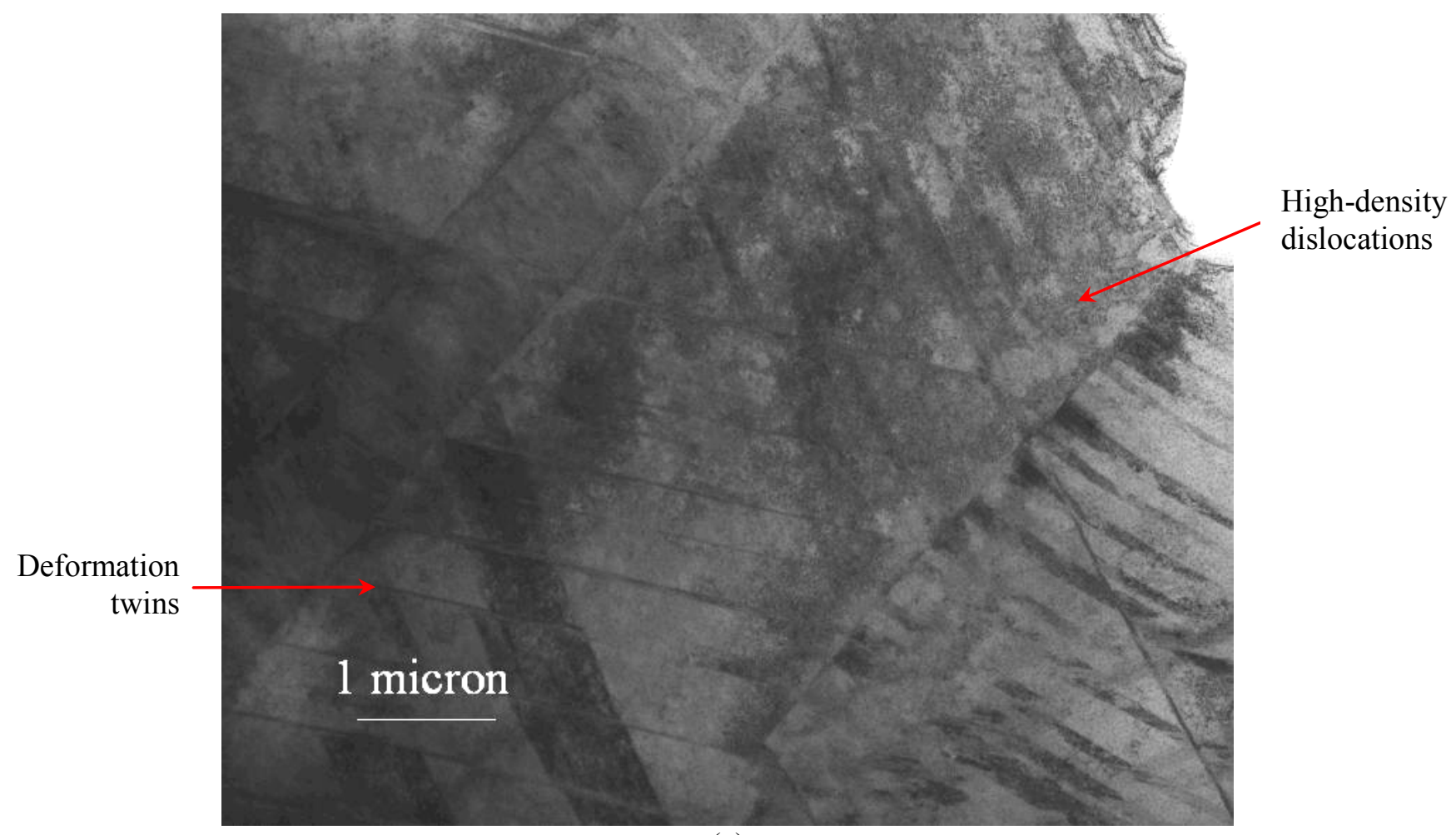

(a)

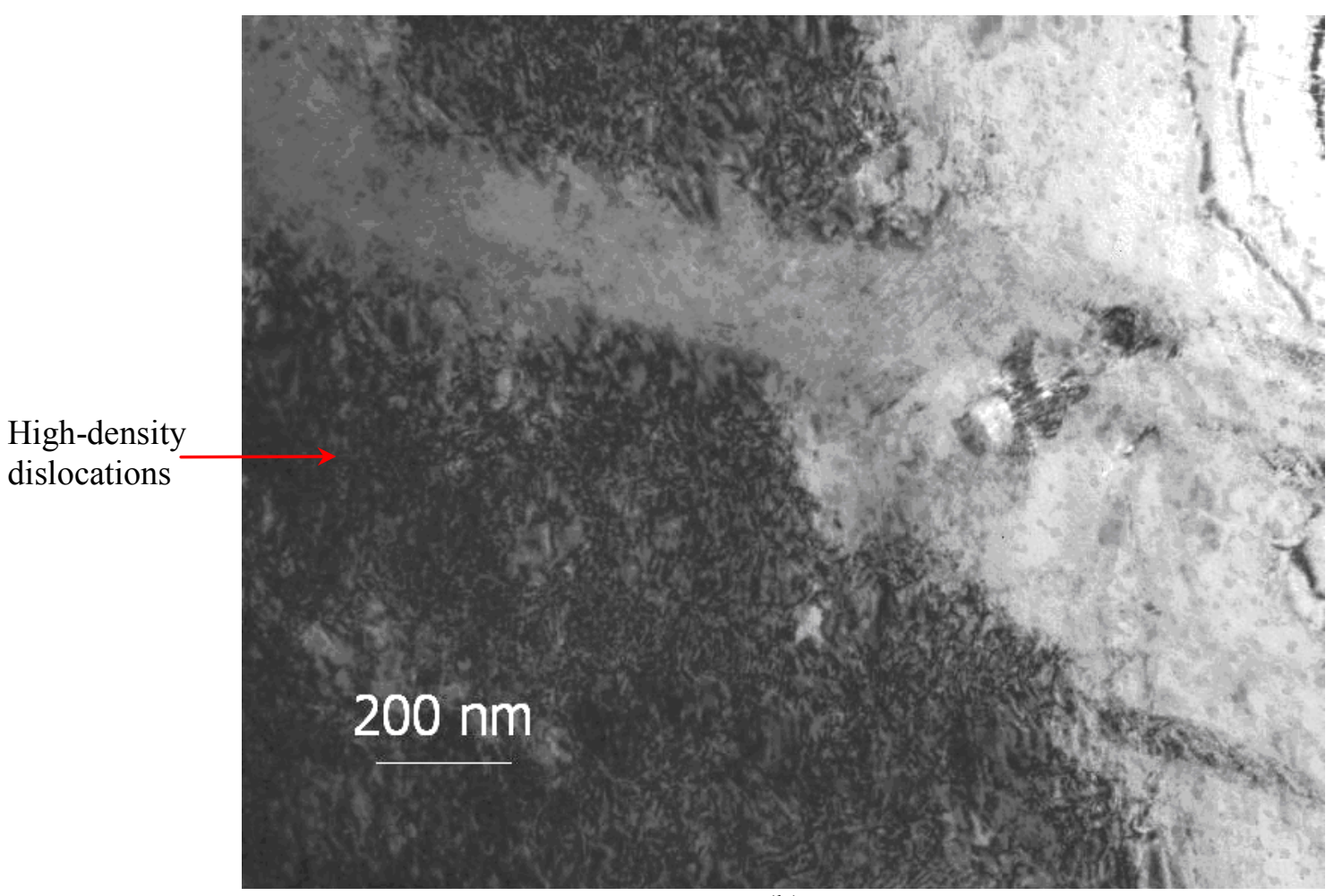

(b)

Figure 8. Nonirradiated microstructure of CW Type 304 SS (Mat. B2): (a) dislocation networks and twins and (b) high density dislocations. 


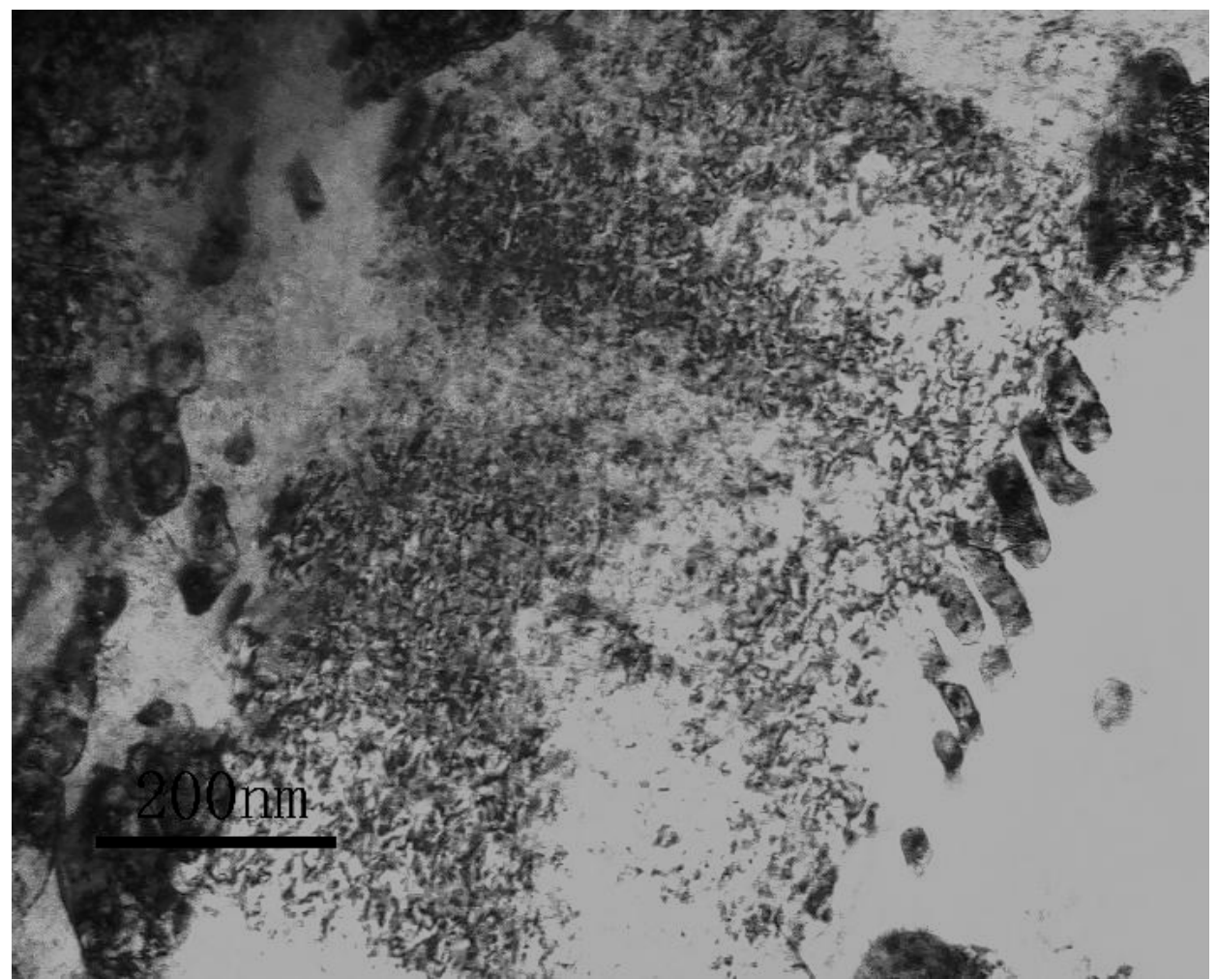

(a)

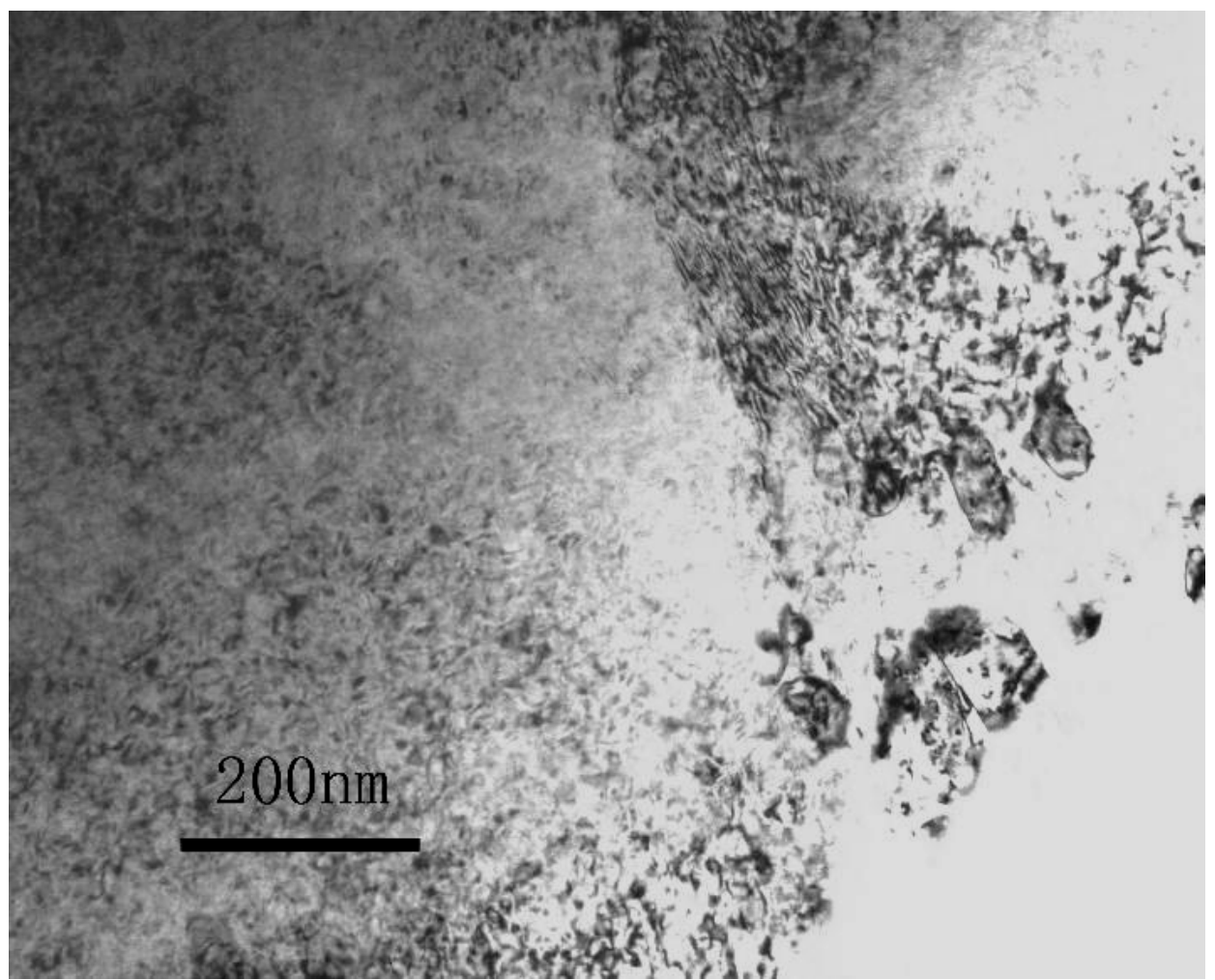

(b)

Figure 9. Irradiated microstructure of CW Type 304 SS (Mat. B2) at $24.5 \mathrm{dpa}$. 


\subsubsection{Solution-Annealed HP 304L SS}

The irradiated microstructure of a SA Type 304L SS (Mat. A8) was examined at two dose levels, 24.5 and $45.0 \mathrm{dpa}$. This material is a high-purity (HP) low-carbon $304 \mathrm{SS}$ with high oxygen content (Table 2). The major defects in the 24.5-dpa and 45-dpa specimens were faulted dislocation loops; no voids were observed. A typical irradiated microstructure is shown by the BF and relrod DF images in Fig. 10. Frank loops characterized with relrod images showed an average loop size around $12 \mathrm{~nm}$ at both doses. The loop densities are $1.2 \times 10^{22} \mathrm{~m}^{-3}$ and $5.3 \times 10^{22} \mathrm{~m}^{-3}$ for 24.5 and $45 \mathrm{dpa}$, respectively. No precipitation was present at both doses.

Table 7. Observations and measurements of HP 304L SS (Mat. A8).

\begin{tabular}{ccccc}
\hline \multirow{2}{*}{ Dose $(\mathrm{dpa})$} & Void & Precipitation & $\begin{array}{c}\text { Average loop size } \\
(\mathrm{nm})\end{array}$ & $\begin{array}{c}\text { Loop density } \\
\left(\mathrm{x} 10^{22} \mathrm{~m}^{-3}\right)\end{array}$ \\
\hline 24.5 & Not observed & Not observed & 12.2 & 1.2 \\
45.0 & Not observed & Not observed & 12.1 & 5.3 \\
\hline
\end{tabular}

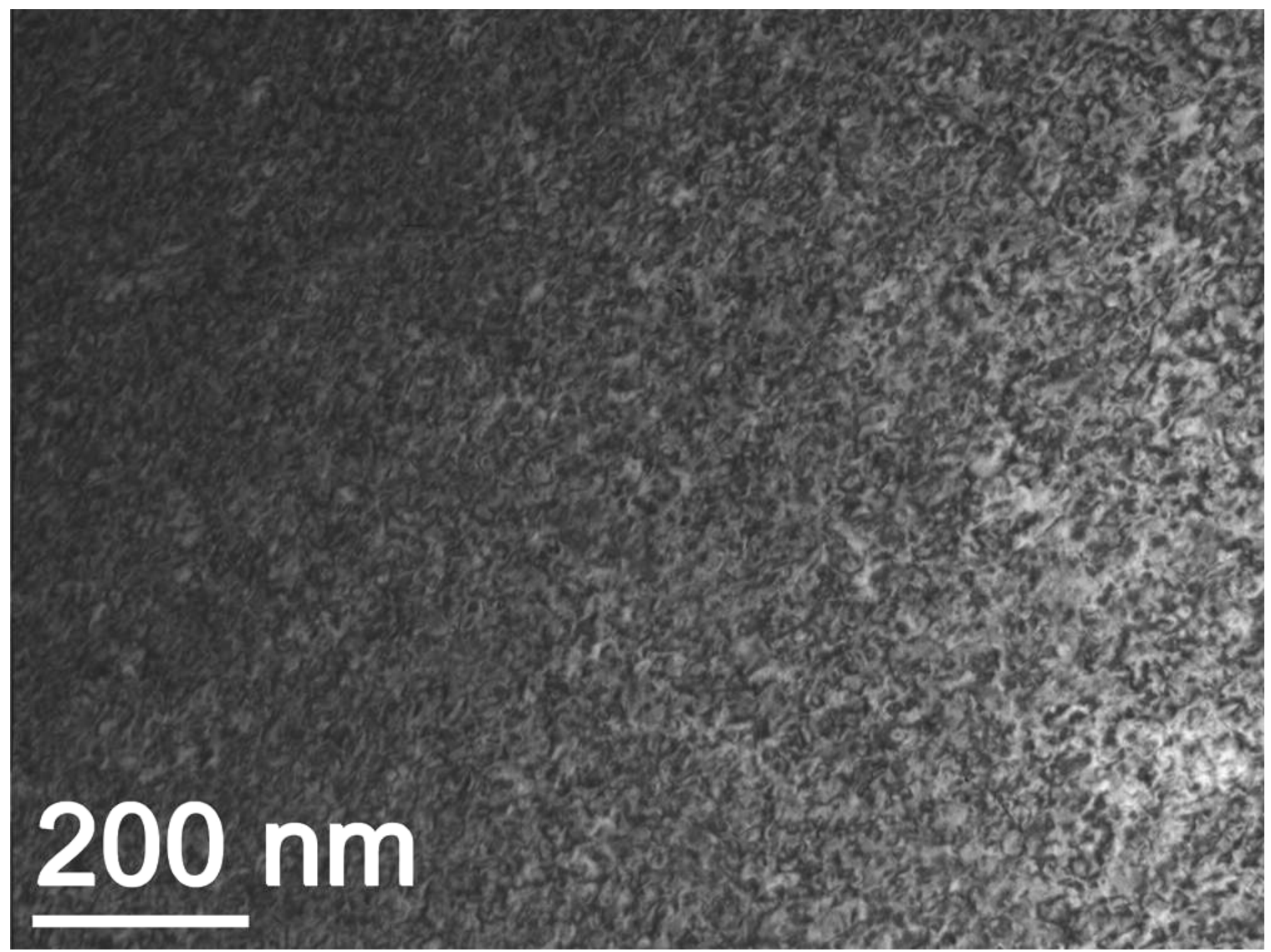

(a)

Figure 10. Irradiated microstructure of SA HP 304L SS (Mat. A8) at 45 dpa: (a) BF image and (b) relrod DF image. 




(b)

Figure 10. (Contd.)

\subsection{Type 347 SS}

Type 347 SS is a variant of Type 304 SS with addition of niobium or tantalum to reduce sensitization. Nickel content is also slightly higher in Type 347 SS. A solution-annealed Type 347 SS (Mat. Code D1 in Table 2) was examined at 24.5 and 45 dpa.

An initial perforation was unsuccessful for the 24.5-dpa specimen, suggesting a slightly different electrochemical polishing condition may be required for this alloy. The electrochemical polishing was repeated before a small thin area was obtained on the 24.5-dpa sample for TEM examination. For the 45-dpa sample, the thin foil quality was improved by the pre-polish dimpling. No voids were observed with the through-focus technique at high magnification, and the irradiated microstructure was dominated by a high density of Frank loops at both doses. Figure 11 shows the irradiated microstructure of the 45-dpa specimen. Small irradiation defects are clearly visible in the BF and relrod DF images. While no precipitation was observed at $24.5 \mathrm{dpa}$, fine precipitates were easily identified in the 45-dpa specimen at grain boundaries (Fig. 11c) and within grains (Fig. 11d). Limited by the beam spot size of TEM, characterization of the fine precipitates was not possible.

Table 8. Observations and measurements of SA Type 347 SS (Mat. D1).

\begin{tabular}{ccccc}
\hline \multirow{2}{*}{ Dose (dpa) } & Void & Precipitation & $\begin{array}{c}\text { Average loop size } \\
(\mathrm{nm})\end{array}$ & $\begin{array}{c}\text { Loop density } \\
\left(\mathrm{x} 10^{22} \mathrm{~m}^{-3}\right)\end{array}$ \\
\hline 24.5 & Not observed & Not observed & 9.4 & 2.0 \\
45.0 & Not observed & Present & 10.8 & 5.9 \\
\hline
\end{tabular}



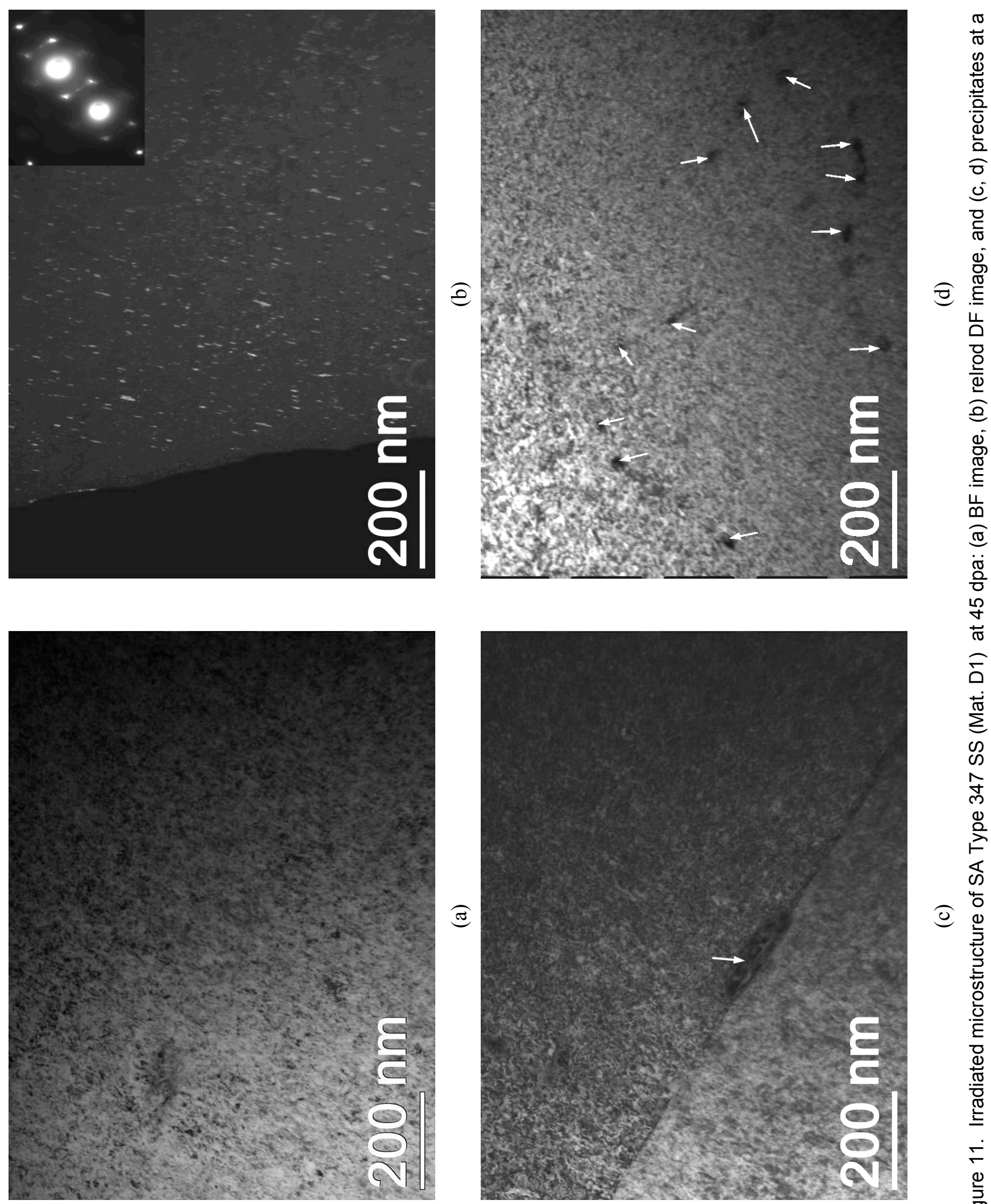

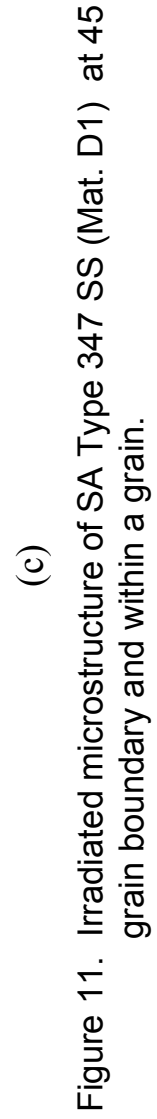




\subsection{Type 316LN SSs}

Two low-carbon high-nitrogen grade 316 SSs with and without titanium addition were included in the study (Mat. Codes B3 and B4 in Table 2). Nitrogen is known to be an austenitic stabilizer and also a solid-solution strengthener. With additional titanium, fine dispersion TiN particles can be formed to further strengthen SSs at elevated temperatures. ${ }^{22}$ Although the two alloys were solution-treated prior to the irradiation, a dislocation cell structure resulting from heavy deformation was still present as shown in Fig. 12 (for Mat. B3). This starting microstructure implies an inadequate solution-anneal treatment, and may complicate the post-irradiation TEM characterization.

Type 316LN SS was examined at all four doses, and Type 316LN-Ti was only observed at $45 \mathrm{dpa}$. Figure 13 shows the irradiated microstructure of Type 316LN SS at 24.5 and $45 \mathrm{dpa}$. Compared with the nonirradiated material (Fig. 12), irradiated defects were clearly visible as "black spot damage" in the BF image at all doses. No voids or cavities can be seen in this alloy at all doses. Fine precipitates were occasionally observed at sub-grainboundaries (Fig. 13b) at 24.5 and $45 \mathrm{dpa}$. The precipitates were probably nitride, but no chemical analysis was performed due to the limitation of the microscope. Despite a heavily deformed matrix, relrod images were obtained at 5.5, 10.2 and 24.5 dpa. The size distributions of Frank loops were measured and are summarized in Table 9. For the 45-dpa specimen, relrod images were not obtained due to highly deformed microstructure. Thus, no quantitative analysis was performed. A comparison of the BF images at 24.5 and 45 dpa (Fig. 13a and c) suggested a similar irradiation defect structure at these doses.

Table 9. Observations and measurements of SA Type 316LN SS (Mat. B3).

\begin{tabular}{ccccc}
\hline \multirow{2}{*}{ Dose (dpa) } & Void & Precipitation & $\begin{array}{c}\text { Average loop size } \\
(\mathrm{nm})\end{array}$ & $\begin{array}{c}\text { Loop density } \\
\left(\times 10^{22} \mathrm{~m}^{-3}\right)\end{array}$ \\
\hline 5.5 & Not observed & Not observed & 8.7 & 3.5 \\
10.2 & Not observed & Not observed & 8.8 & 5.8 \\
24.5 & Not observed & Present & 5.8 & 1.7 \\
45.0 & Not observed & Present & Not quantified & Not quantified \\
\hline
\end{tabular}

The irradiated microstructure of Type 316LN-Ti SS is shown in Fig. 14. Titanium addition does not have any effect on the irradiated microstructure at $45 \mathrm{dpa}$. Frank loops were still the dominant irradiation defects, and no voids or cavities were evident in this alloy. While no high-density, dispersed secondphase particles were observed, fine precipitates were present in this alloy. Because of the heavily deformed matrix, relrod DF analysis was not performed. 


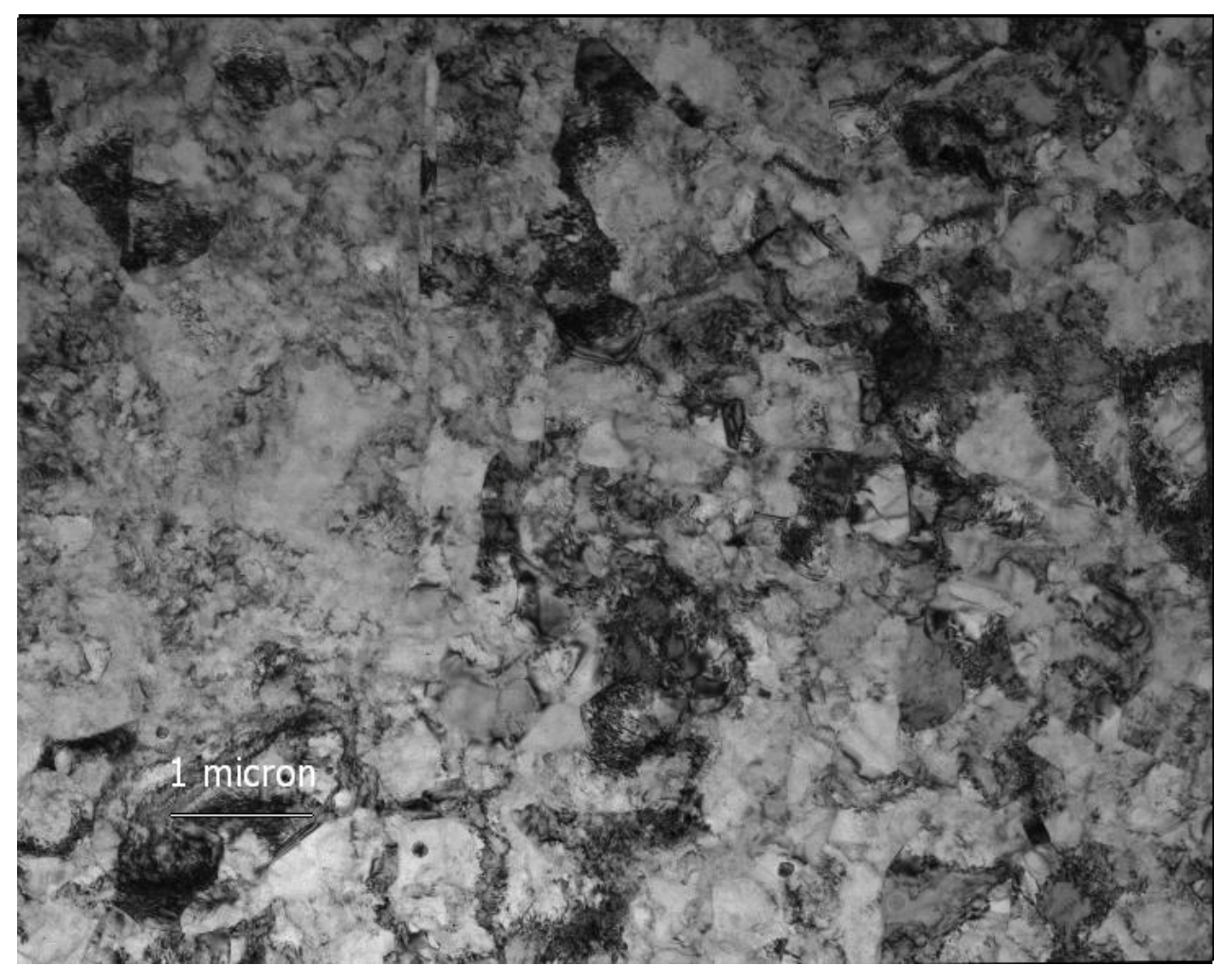

(a)

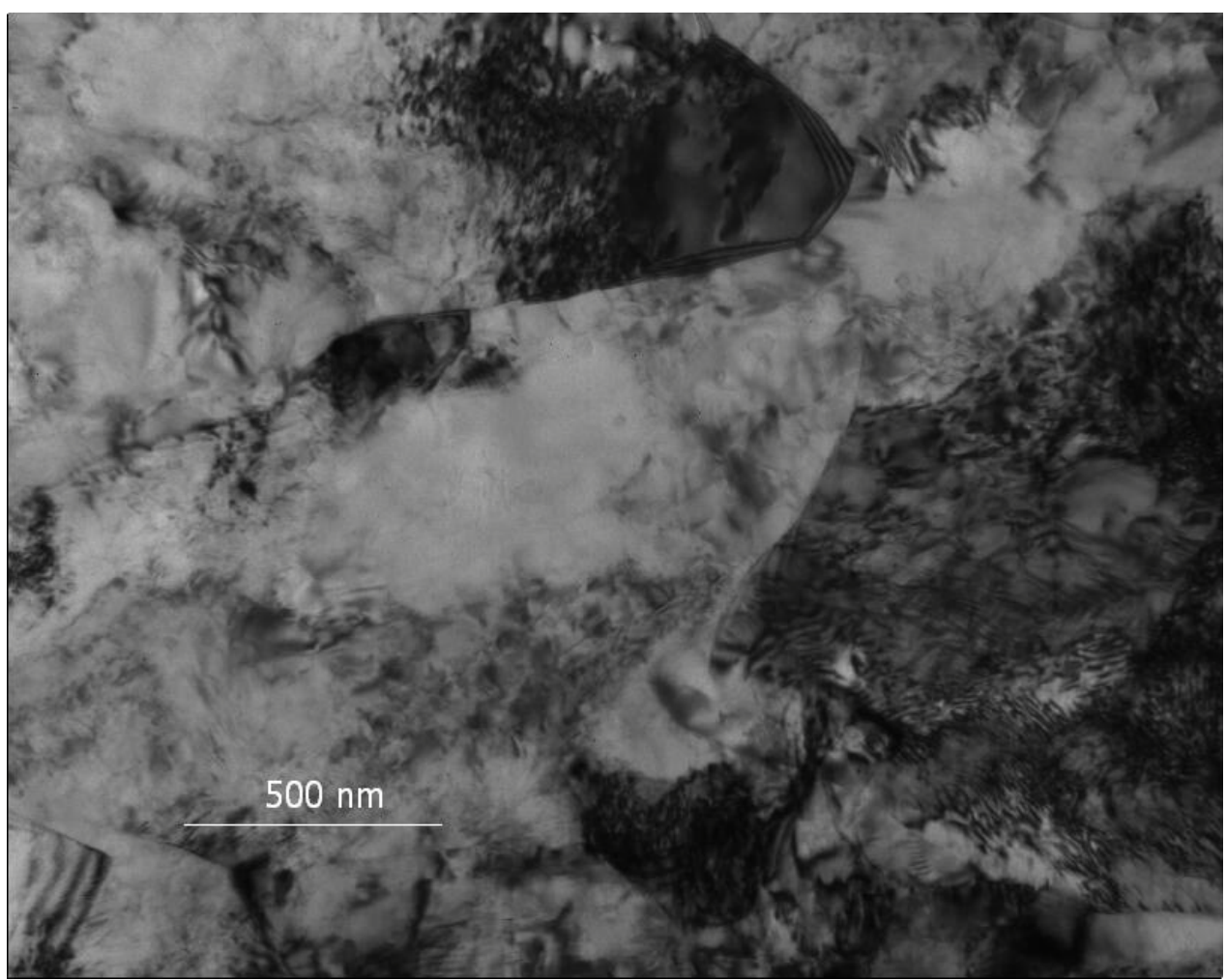

(b)

Figure 12. Nonirradiated microstructure of Type 316LN SS (Mat. B3). 

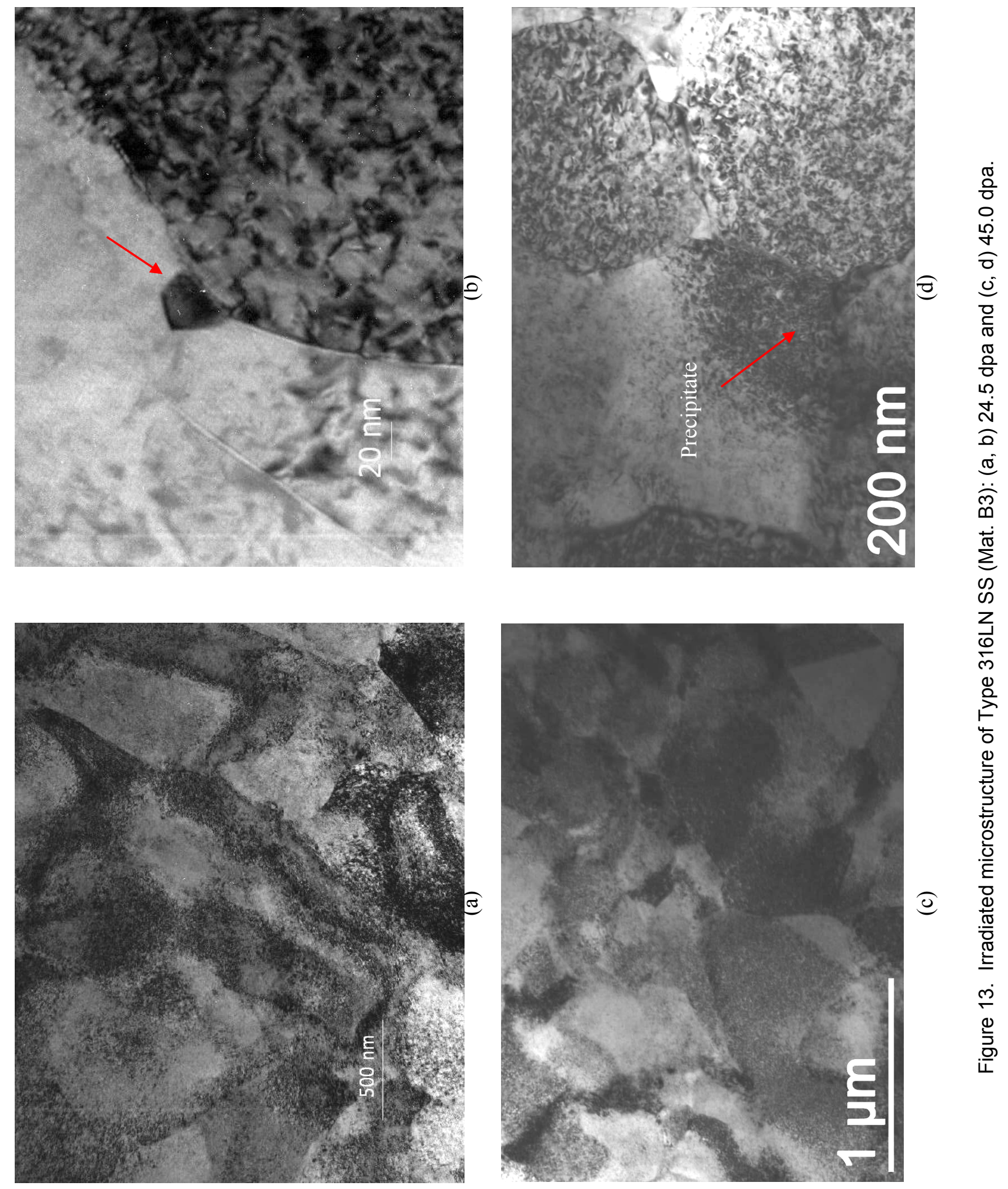

$24.5 \mathrm{dpa}$

45.0 dpa 


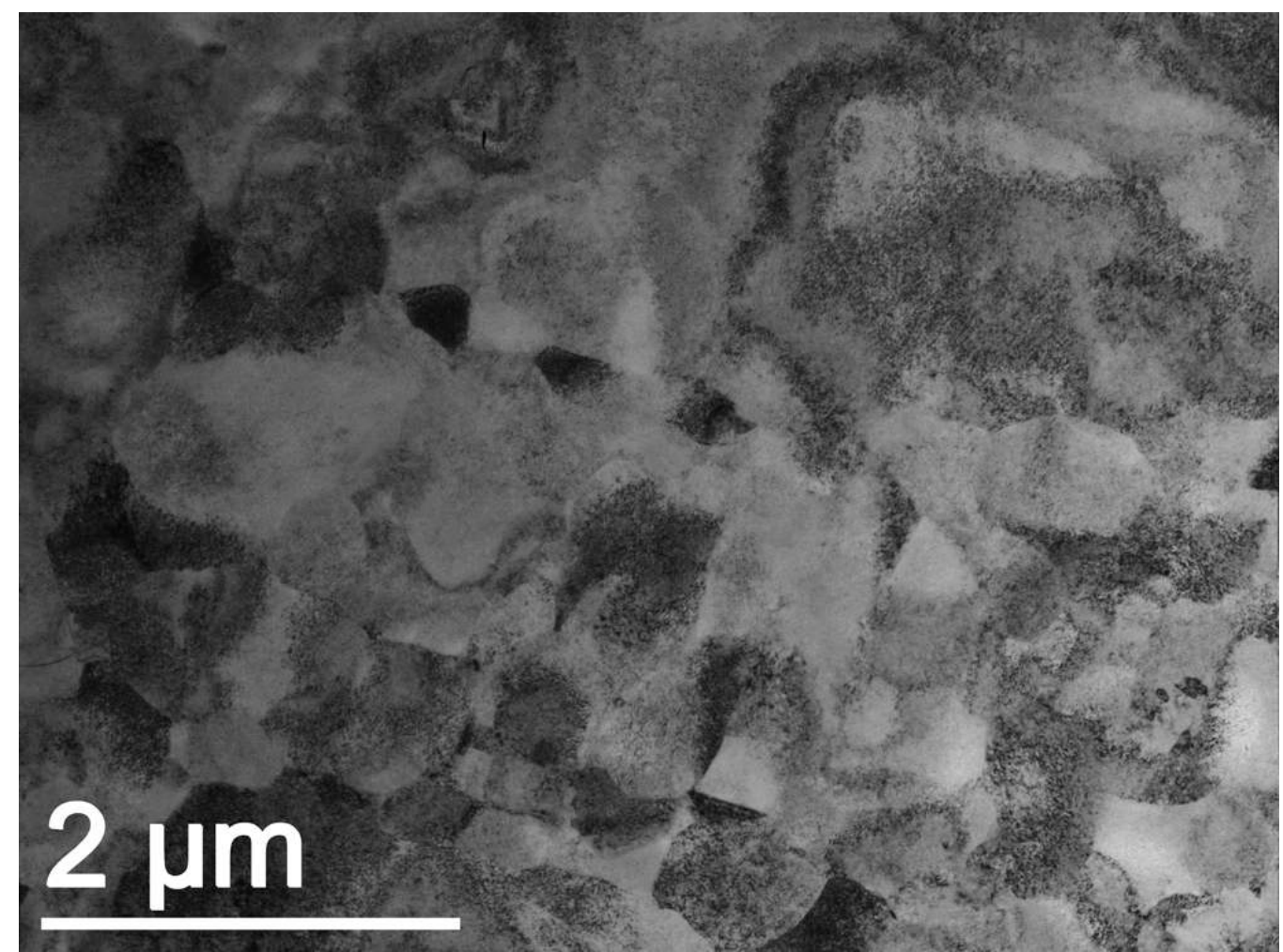

(a)

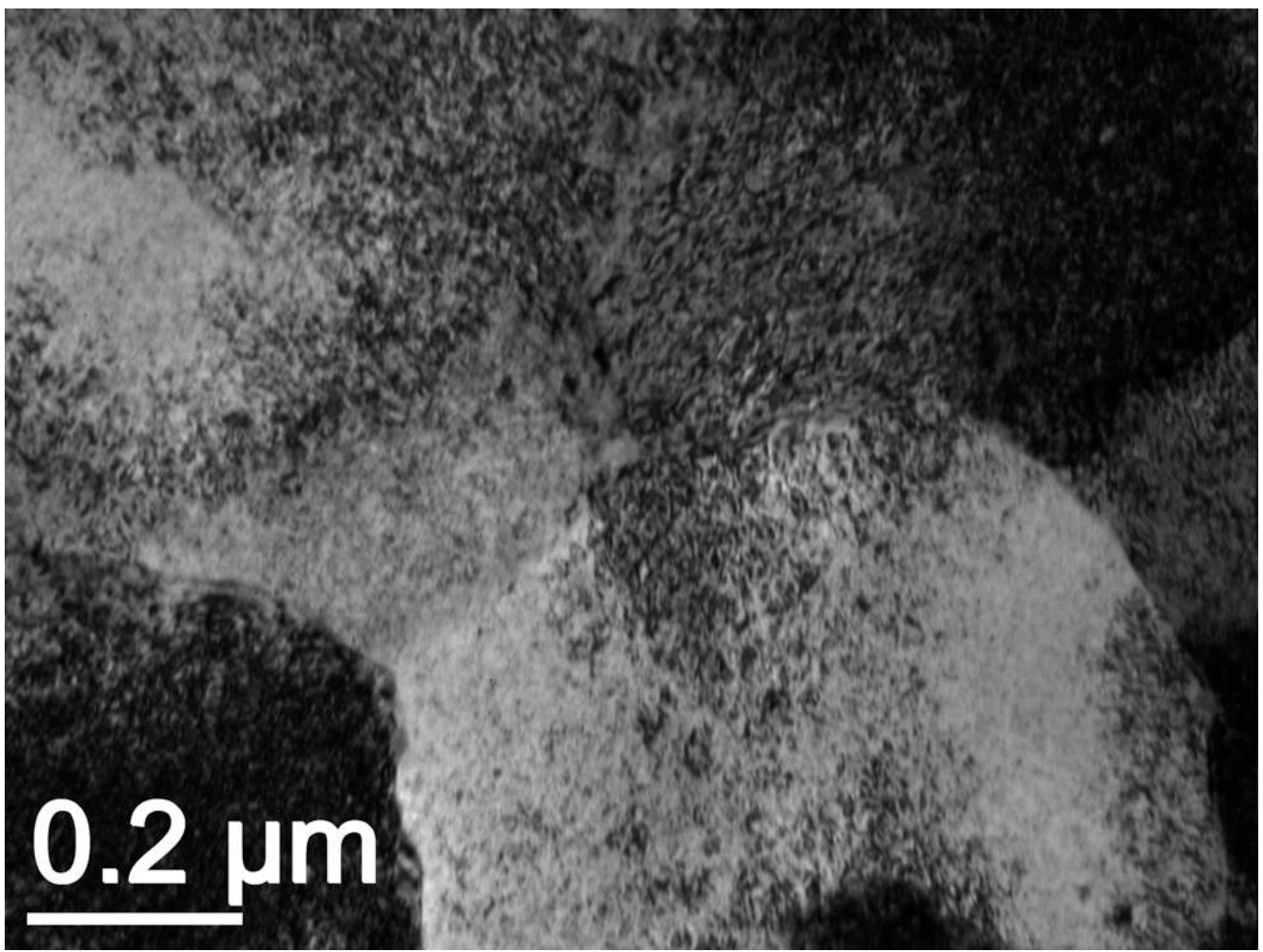

(b)

Figure 14. Irradiated microstructure of Type 316LN-Ti SS (Mat. B4) at $45 \mathrm{dpa}$. 


\subsection{Cast SSs}

Three cast SSs with different carbon and ferrite contents were examined at 24.5 and 45 dpa in this study. One of the cast materials was a CF-3 grade SS with 14\% ferrite (Mat. Code C1), and the other two alloys were CF-8 grade SSs with $14 \%$ and $24 \%$ ferrite contents (Mat. Codes C2 and C4, respectively). The initial cast microstructure was nearly free of any dislocations as shown in Fig. 15. Ferrite phase was readily identified in the austenite matrix as island bands.



Figure 15. Nonirradiated microstructure of CF-8 cast SS (Mat. C4).

\subsubsection{CF-3 Cast Stainless Steel}

Irradiated microstructures of CF-3 are shown in Figs. 16 and 17 for 24.5- and 45-dpa specimens, respectively. The austenite-ferrite duplex microstructure was unaffected by neutron exposure, and irradiation defects appeared in both phases. While relrod DF images were used to visualize irradiation defects in the austenitic phase (Fig. 16b), weak-beam dark field (WBDF) conditions were applied to image defects in the ferrite (e.g. Fig. 16c). Compared to the austenitic phase, irradiation defects seemed less dense and smaller in the ferrite.

Irradiated microstructures were also similar at 24.5 and 45 dpa. As shown in Fig. 17, irradiation defects appear in the cast duplex microstructure at $45 \mathrm{dpa}$. The average size of the Frank loops revealed by the relrod images was similar at the two doses, but the loop density appears higher in the 45-dpa sample.

Fine precipitates in the austenite were seen in the 45-dpa specimen. Using the through-focus technique, the samples were examined for voids under high-magnification. No voids or cavities were evident in the austenitic or ferrite phases at $45 \mathrm{dpa}$. 


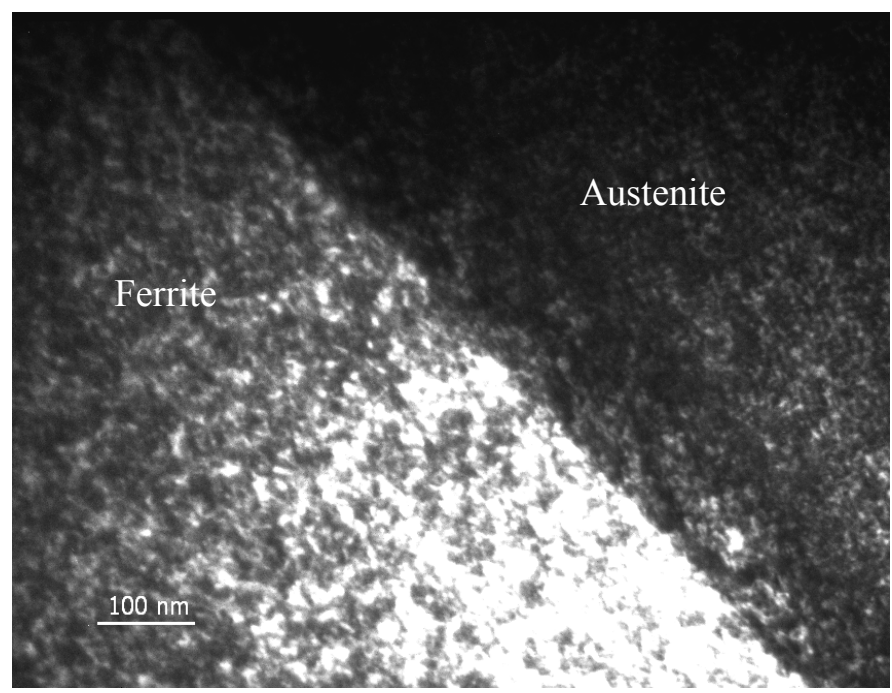

(a)

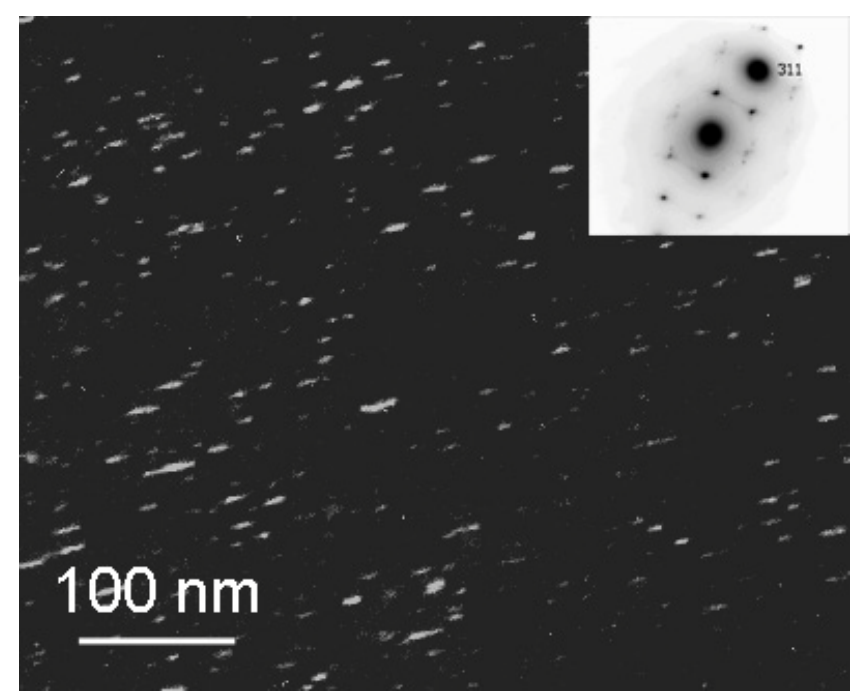

(b)

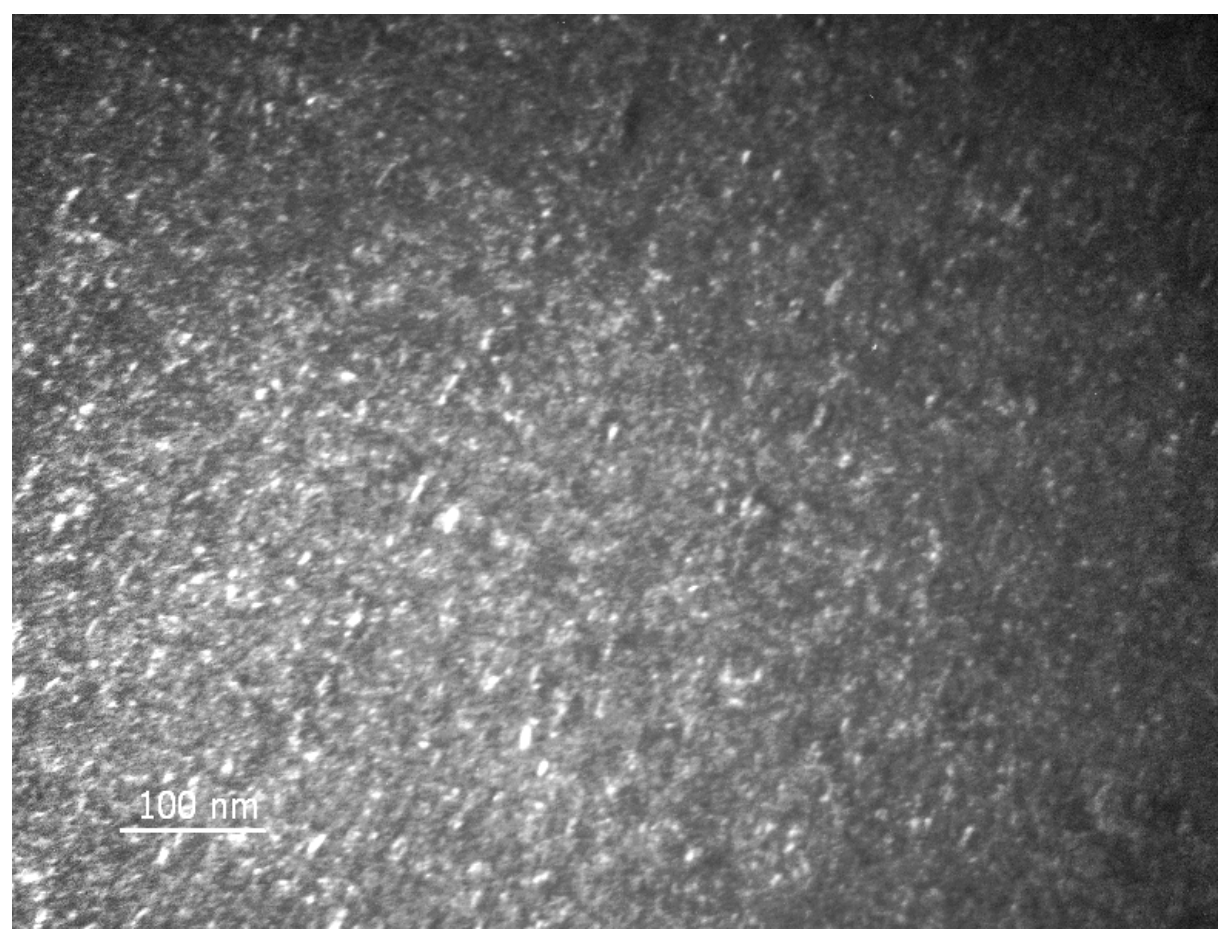

(c)

Figure 16. Irradiated microstructure of CF-3 with 14\% ferrite (Mat. C1) at 24.5 dpa: (a) general view of duplex structure, (b) relrod DF images showing Frank loops in austenitic phase, and (c) WBDF image of irradiation defects in ferrite. 


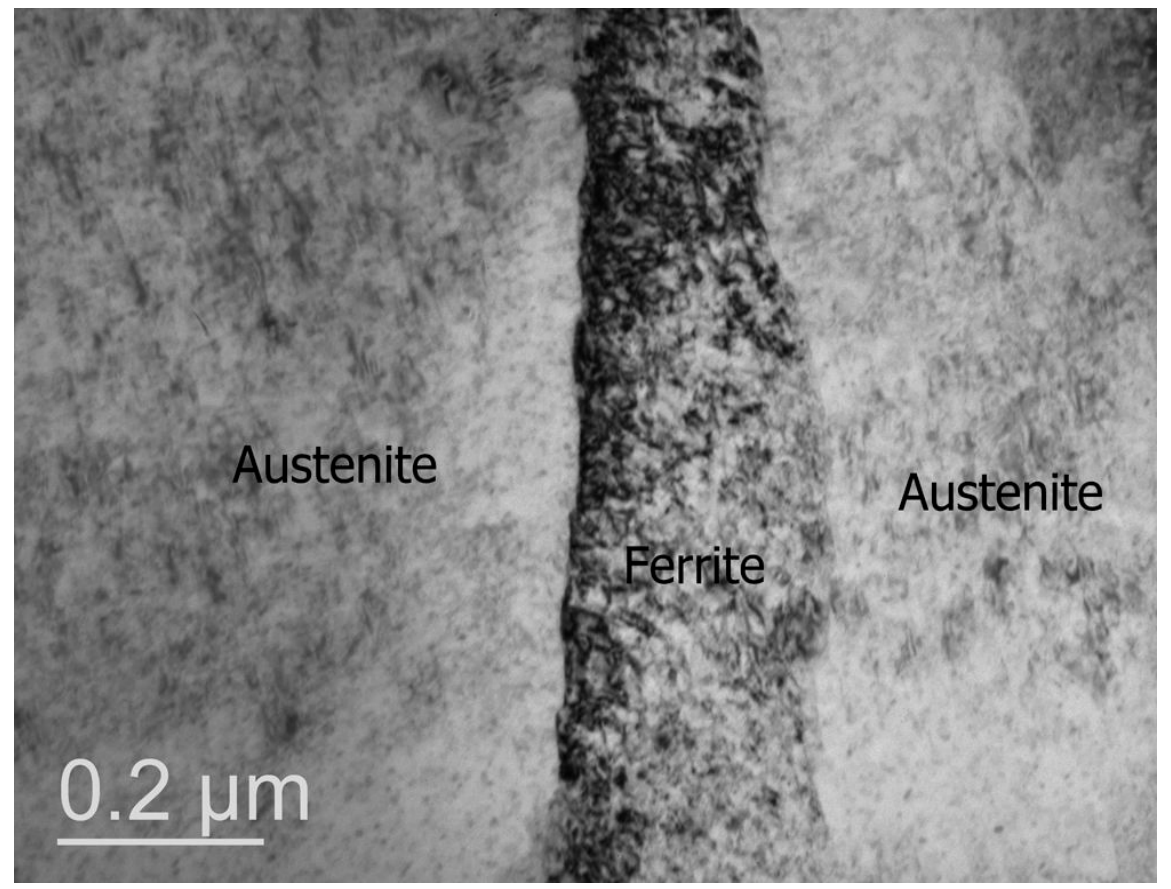

(a)

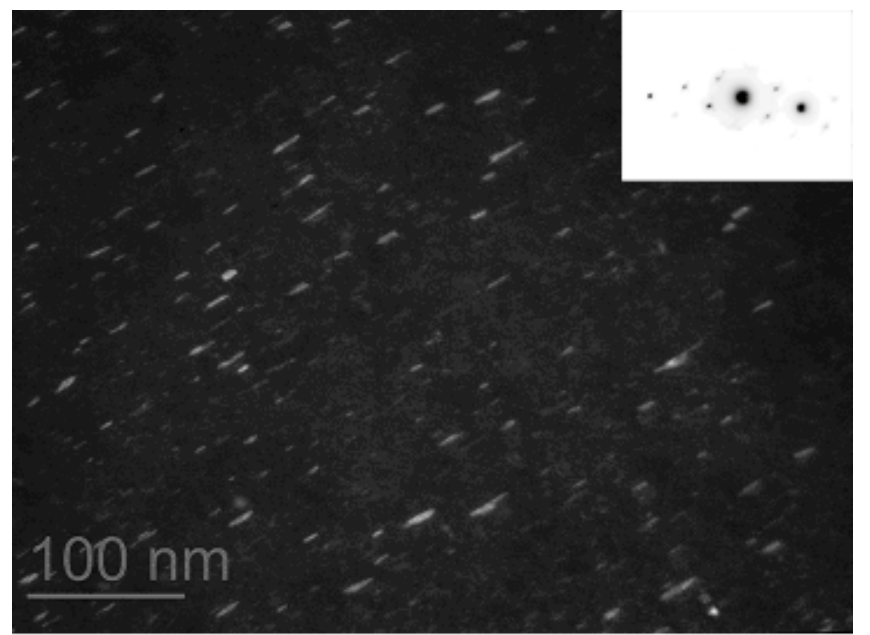

(b)



(c)

Figure 17. Irradiated microstructure of CF-3 with $14 \%$ ferrite (Mat. C1) at 45 dpa: (a) general duplex microstructure, (b) relrod DF image showing Frank loops in austenite, and (c) precipitates in austenite. 


\subsubsection{CF-8 Cast Stainless Steels}

Two CF-8 grade SSs with different ferrite contents (Mat. Codes C2 and C4) were included in the study. Figures 18 and 19 show their irradiated microstructures at 24.5 and 45 dpa, respectively. Irradiation defects were almost identical between the low-C grade CF-3 (Figs. 16 and 17) and high-C grade CF-8. The density of Frank loops increased with dose from 24.5 dpa to 45 dpa in both CF-3 and CF-8. No voids or cavities were apparent in the austenite or ferrite in images obtained using the through-focus technique under high magnification.

Precipitates were not seen at 24.5 dpa but appeared in the 45-dpa specimen. Using ultra-reflections, precipitates in the austenite were visualized in DF images (see Fig. 20). The density of the precipitates was much higher in CF-8 than in CF-3 at 45 dpa. A higher carbon content may contribute to the development of precipitates in the CF-8 grade cast SS. Table 10 summarizes the TEM results on all cast SSs examined.

Table 10. Observations and measurements of cast SSs (Mats. C1, C2, and C4).

\begin{tabular}{|c|c|c|c|c|c|c|}
\hline \multirow{2}{*}{$\begin{array}{l}\text { Alloy / Mat. } \\
\text { Code }\end{array}$} & \multirow{2}{*}{$\begin{array}{l}\text { Dose } \\
(\mathrm{dpa})\end{array}$} & \multirow{2}{*}{ Void } & \multicolumn{2}{|c|}{ Precipitates } & \multicolumn{2}{|c|}{ Frank loops } \\
\hline & & & Density $\left(\mathrm{x} 10^{20} \mathrm{~m}^{-3}\right)$ & Size (nm) & Density $\left(\mathrm{x} 10^{22} \mathrm{~m}^{-3}\right)$ & Size (nm) \\
\hline \multirow[t]{2}{*}{$\mathrm{CF}-3 / \mathrm{C} 1$} & 24.5 & Not observed & Not observed & - & 2.8 & 8.5 \\
\hline & 45.0 & Not observed & 0.72 & 34 & 6.2 & 10.1 \\
\hline \multirow[t]{2}{*}{$\mathrm{CF}-8 / \mathrm{C} 2$} & 24.5 & Not observed & Not observed & - & 3.1 & 7.7 \\
\hline & 45.0 & Not observed & 73 & 6.7 & 9.2 & 9.0 \\
\hline $\mathrm{CF}-8 / \mathrm{C} 4$ & 24.5 & Not observed & - & - & 1.7 & 16.3 \\
\hline
\end{tabular}




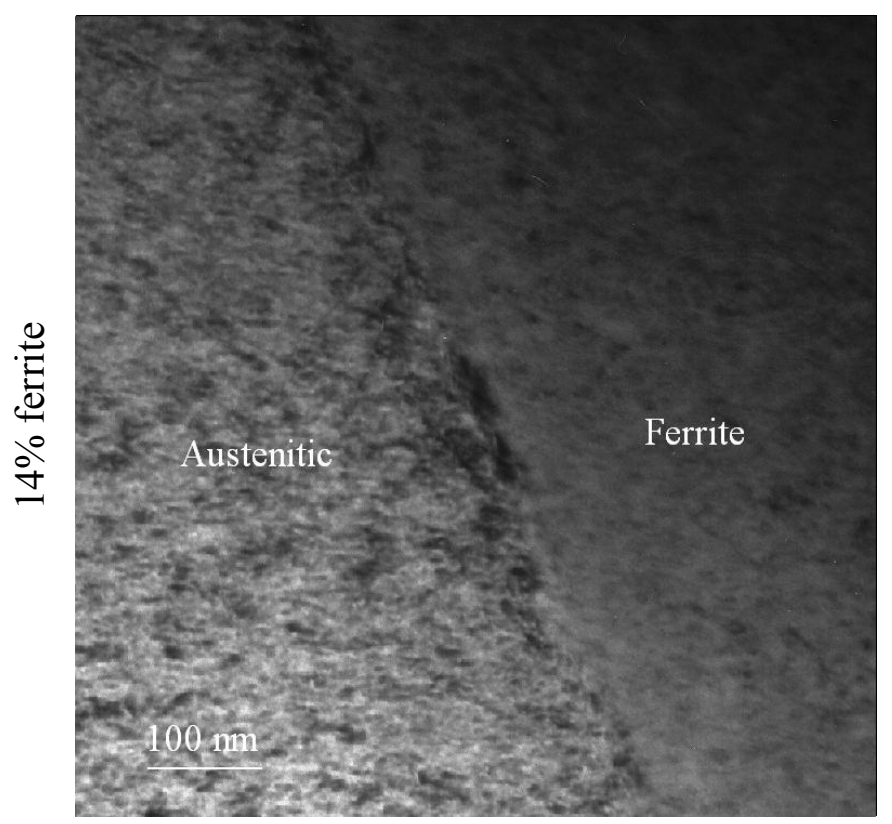

(a)



(c)

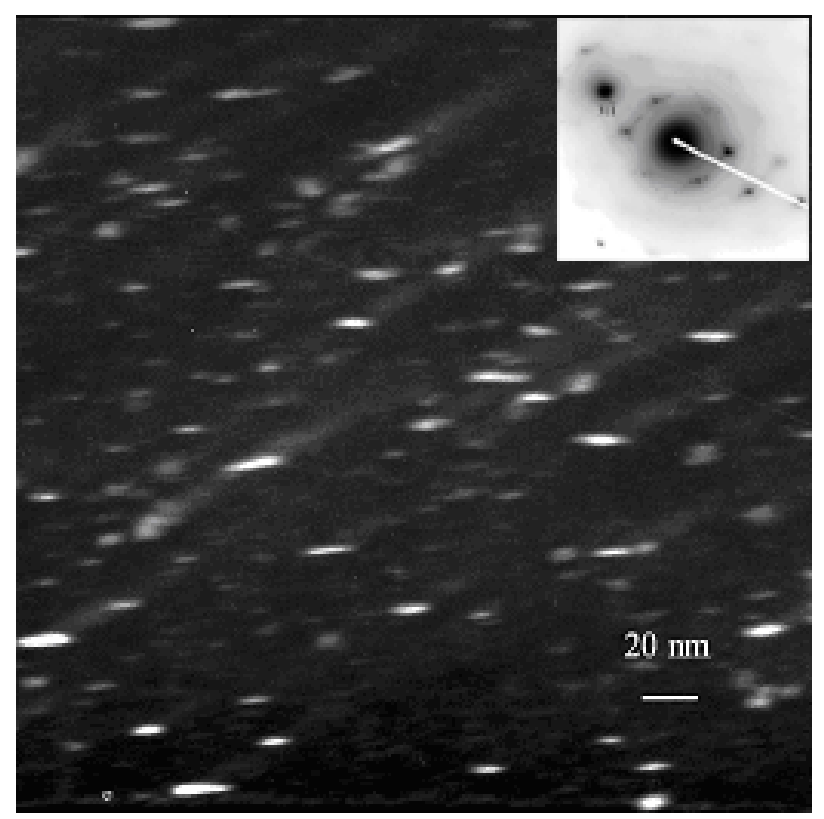

(b)

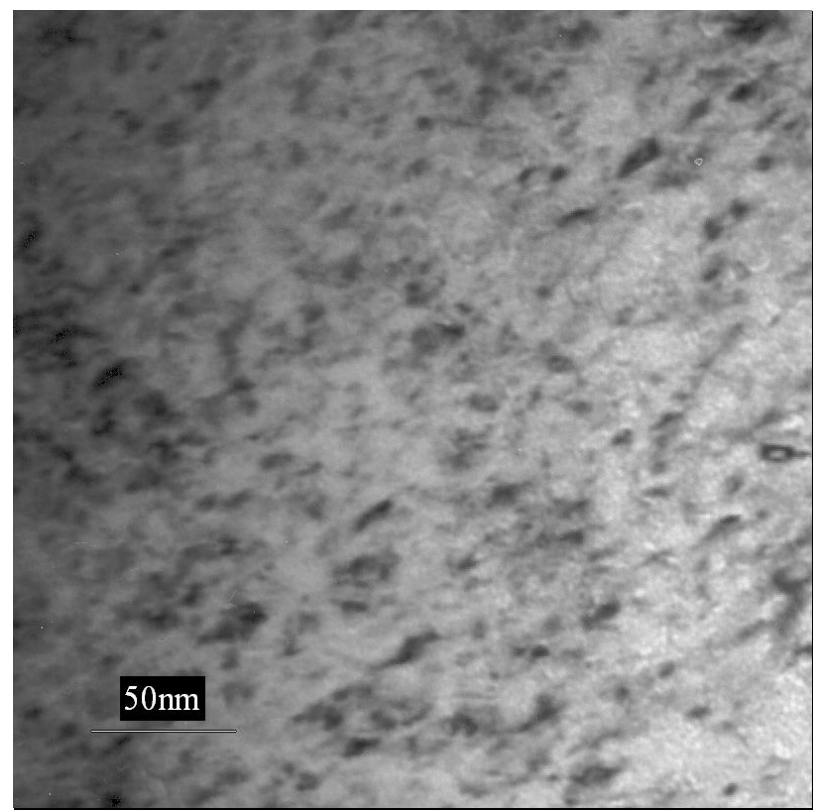

(d)

Figure 18. Irradiated microstructure of CF-8 (Mats. C2 and C4) at 24.5 dpa: (a) general view of duplex microstructure of Mat. C2, (b) relrod DF image showing Frank loops in austenitic phase in Mat. C2, (c) irradiation defects in austenite in Mat. C4, and (d) irradiation defects in ferrite in Mat. C4. 




(a)



(b)

Figure 19. Irradiated microstructure of CF-8 with $14 \%$ ferrite at 45 dpa (Mat. C2): (a) general view of duplex microstructure and (b) relrod DF image of Frank loops in austenite. 


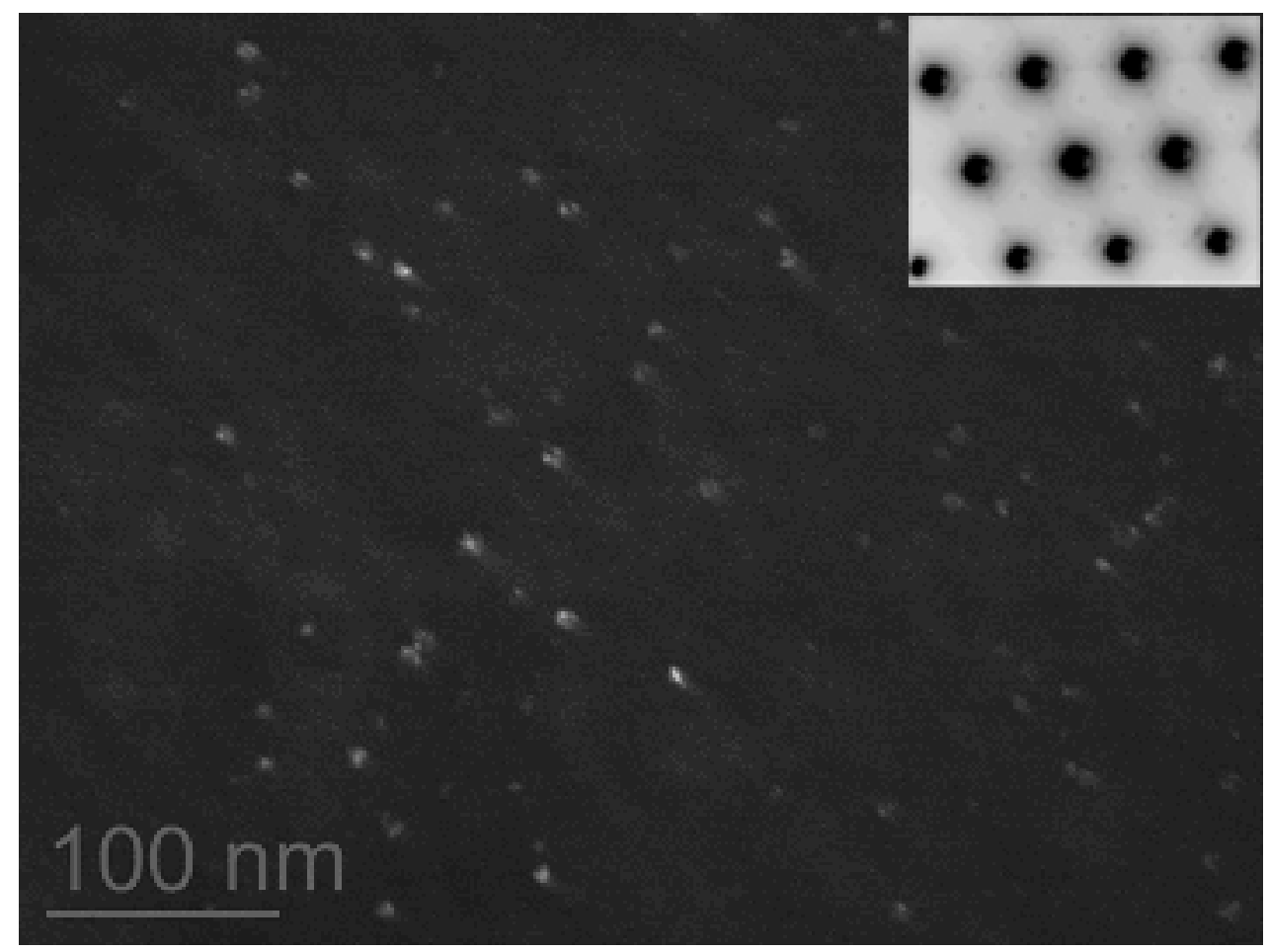

Figure 20. Fine precipitates in austenite of CF-8 at 45 dpa visualized in a DF image with ultra-reflection. 


\subsection{Nickel Alloys}

Void swelling and irradiation defect structure were also studied in two Alloy 690s with and without grainboundary-engineering (GBE) treatment (Mat. Codes E1 and E2). Figure 21 shows the nonirradiated microstructure of the two alloys. Both of them contained a high density of twin bands and had many precipitates mainly distributed at grain boundaries. The precipitates were identified as $\mathrm{M}_{23} \mathrm{C}_{6}$ chromiumrich carbides that have a face-centered cubic structure as shown in Fig. 22. The size and distribution of the carbides varied at different grain boundaries. The nonirradiated microstructures were free of dislocations in both GBE and non-GBE alloys.

The irradiated microstructure of Alloy 690 is shown in Figs. 23-25. A few cavities were evident in the 24.5-dpa specimens, as indicated by the arrows in Figs. 23a and 24a. The cavities were scattered throughout the materials and were mainly associated with grain boundaries or close to precipitates. The size of the cavities varied from $\sim 25$ to $\sim 65 \mathrm{~nm}$, similar to the size of the small precipitates apparent in these alloys. These cavities seemed unrelated to irradiation since no cavities were visible in the 45-dpa specimen (Fig. 25). Most likely, the cavities were caused by fall-off of precipitates during electrochemical polishing.

Faulted dislocation loops were the dominant defects in the irradiated microstructure at both doses. For the 24.5 dpa specimens, irradiated defects were compared for Alloy 690 with and without GBE treatment. Based on relrod images, the loop density was comparable for the two alloys, but the loop mean size in the GBE 690 was smaller than that in the Alloy 690. The GBE treatment, which includes a series of deformation-annealing cycles, seems to affect the development of dislocation loops at 24.5 dpa. Table 11 summarizes the TEM results of irradiated nickel alloys.

Table 11. Observations and measurements of Alloy 690s (Mats. E1 and E2).

\begin{tabular}{|c|c|c|c|c|c|}
\hline $\begin{array}{l}\text { Alloy / Mat. } \\
\text { Code }\end{array}$ & Dose (dpa) & Void & Precipitation $^{\mathrm{a}}$ & $\begin{array}{l}\text { Average loop size } \\
\text { (nm) }\end{array}$ & $\begin{array}{l}\text { Loop density } \\
\left(\mathrm{x} 10^{22} \mathrm{~m}^{-3}\right)\end{array}$ \\
\hline \multirow[t]{2}{*}{ GBE 690, E1 } & 24.5 & Not observed $\mathrm{b}$ & N/A & 17 & 1.4 \\
\hline & $45.0^{\mathrm{c}}$ & Not observed & N/A & - & - \\
\hline Alloy 690, E2 & 24.5 & Not observed $\mathrm{b}$ & N/A & 28.9 & 1.1 \\
\hline
\end{tabular}

a Carbides were present both before and after irradiation; however, irradiation-induced precipitation was not analyzed.

$\mathrm{b}$ Cavities resulting from fall-off of precipitates unrelated to irradiation were observed.

c The polished TEM foil was too thick to form a good relrod image for this sample. Thus, the loop size and density were not measured. 


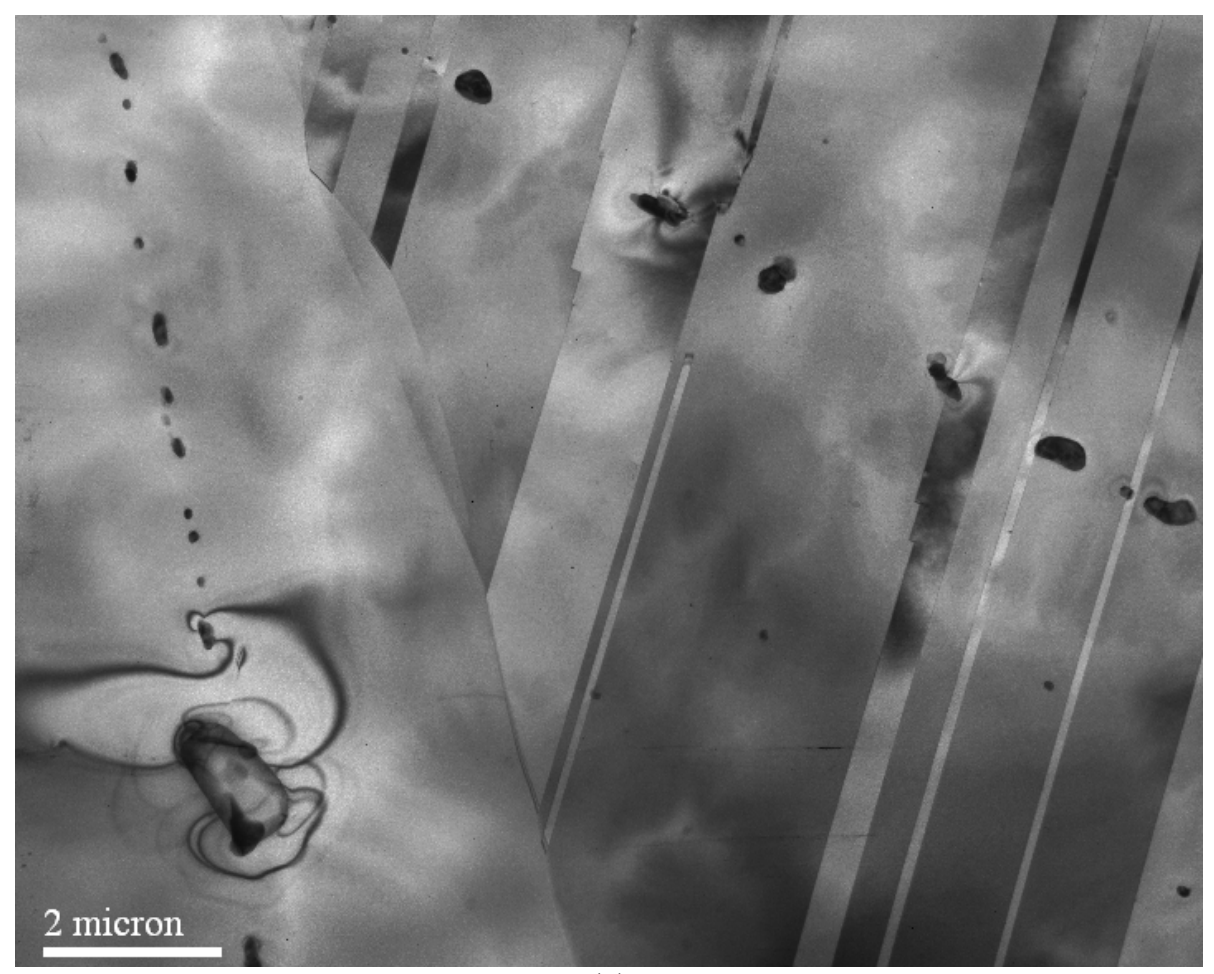

(a)

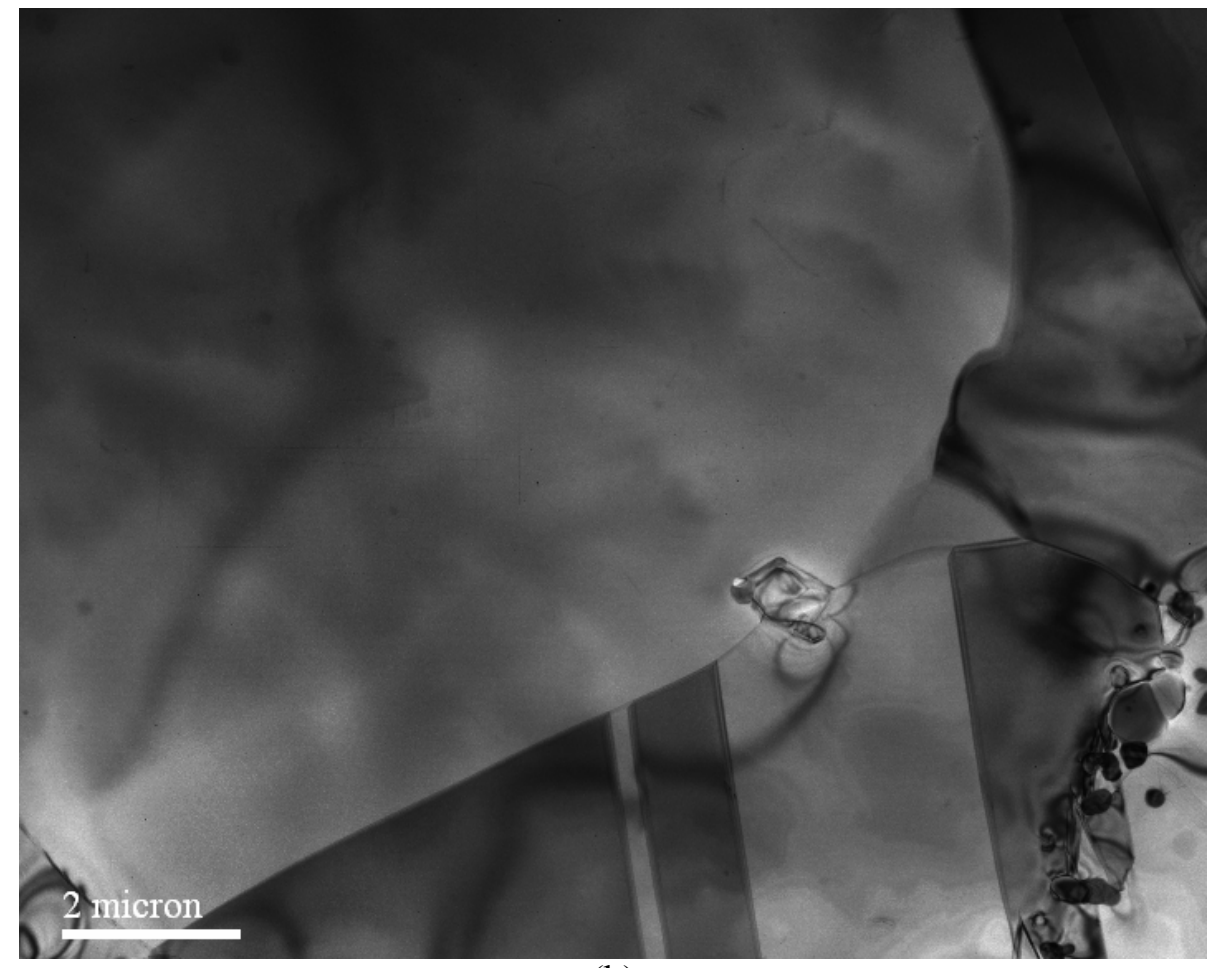

(b)

Figure 21. Nonirradiated microstructure of nickel alloys (Mat. E1 and E2): (a, b) GBE 690 and (c, d) Alloy 690 . 


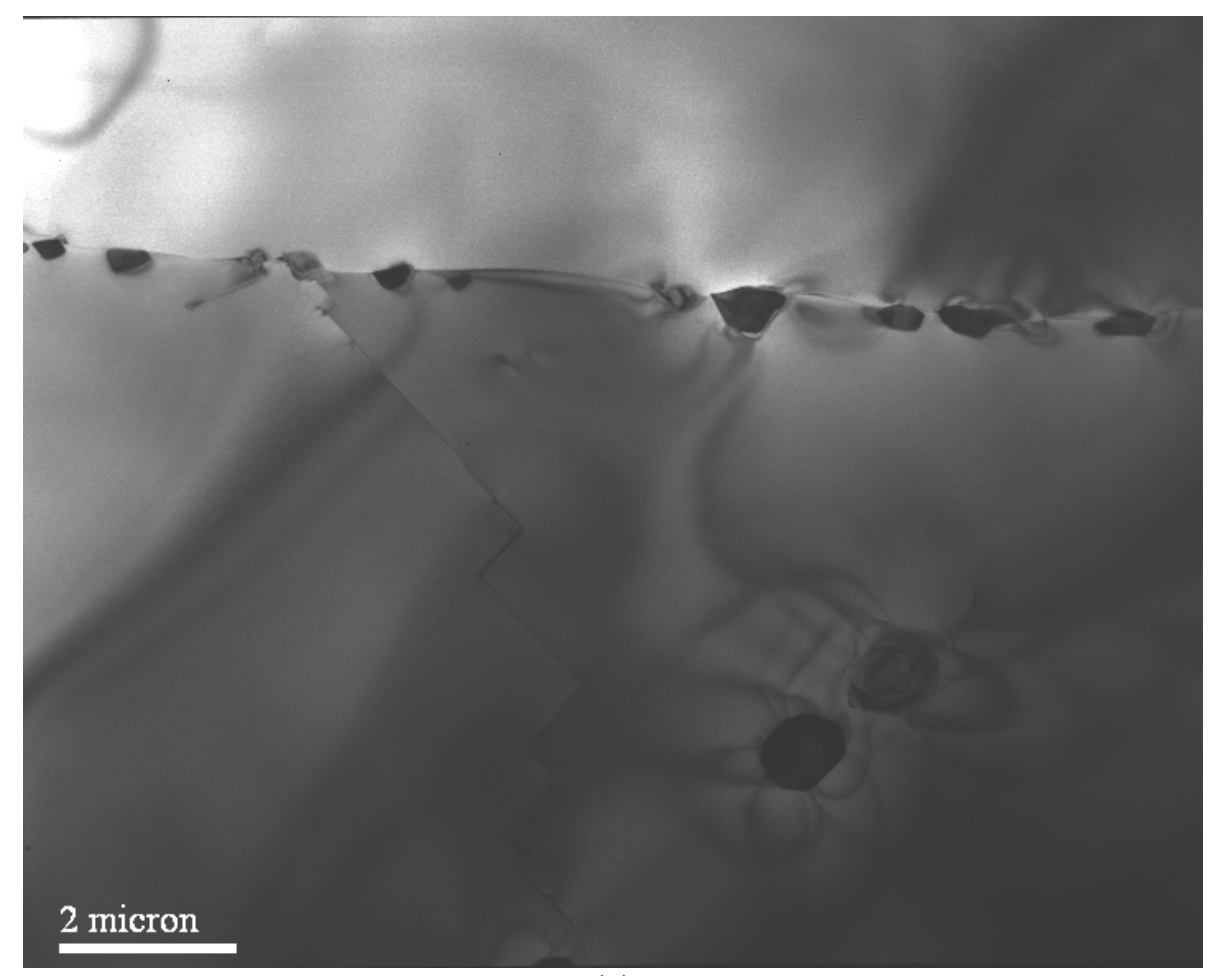

(c)



(d)

Figure 21. (Contd.) 


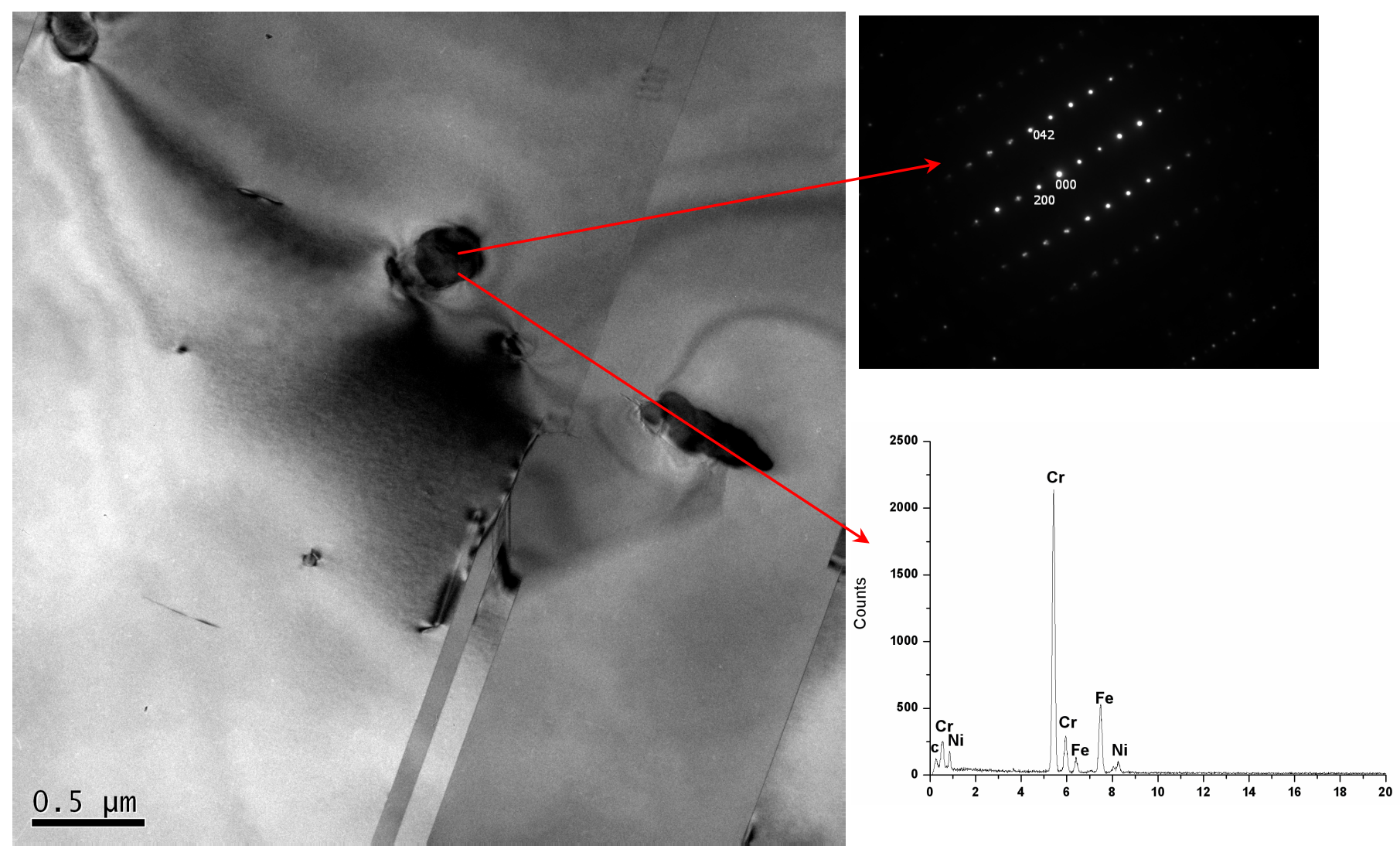

Figure 22. Diffraction pattern and EDS spectrum of $\mathrm{M}_{23} \mathrm{C}_{6}$ precipitate in nonirradiated Alloy 690 . 


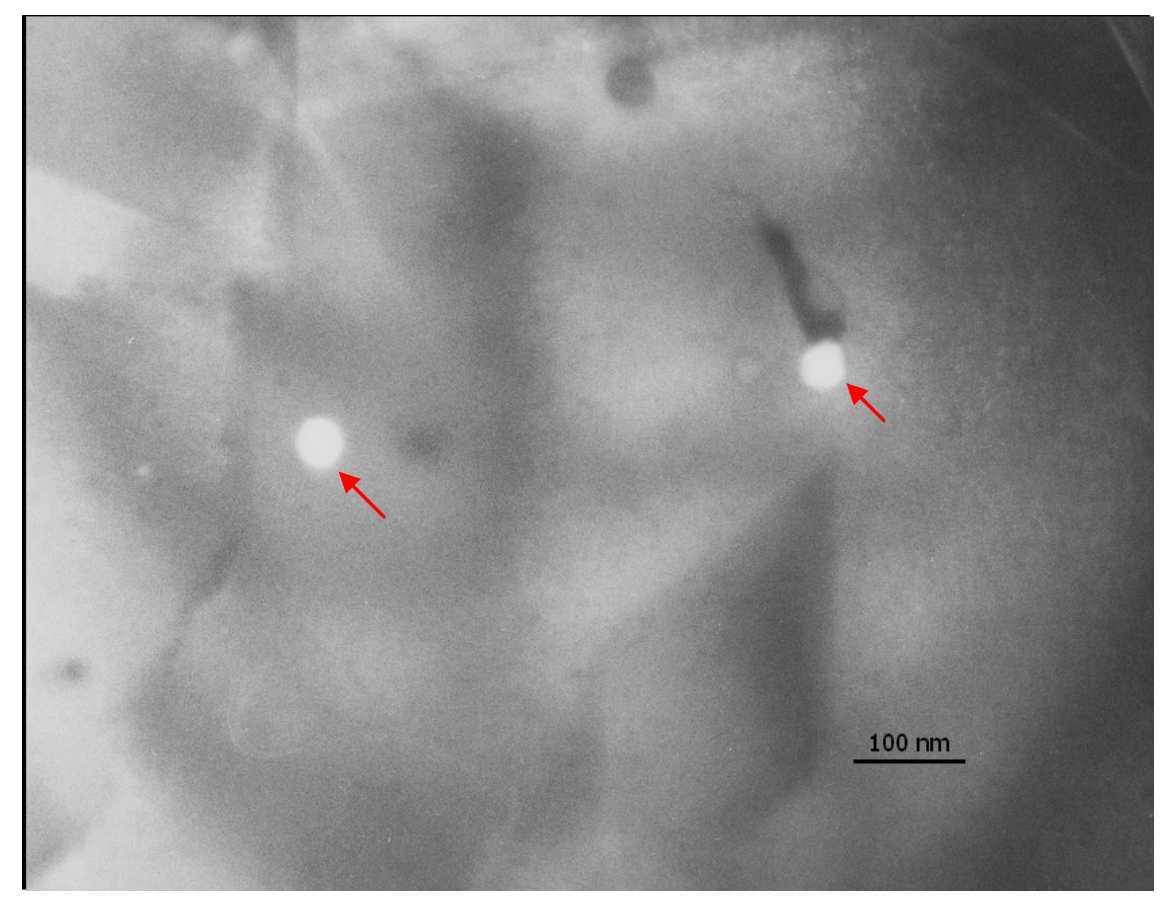

(a)



(b)

Figure 23. Irradiated microstructure of GBE 690 (Mat. E1) at 24.5 dpa: (a) cavities possibly left from falloff of precipitates, (b) BF image of irradiation defects, and (c) relrod DF image of dislocation loops. 


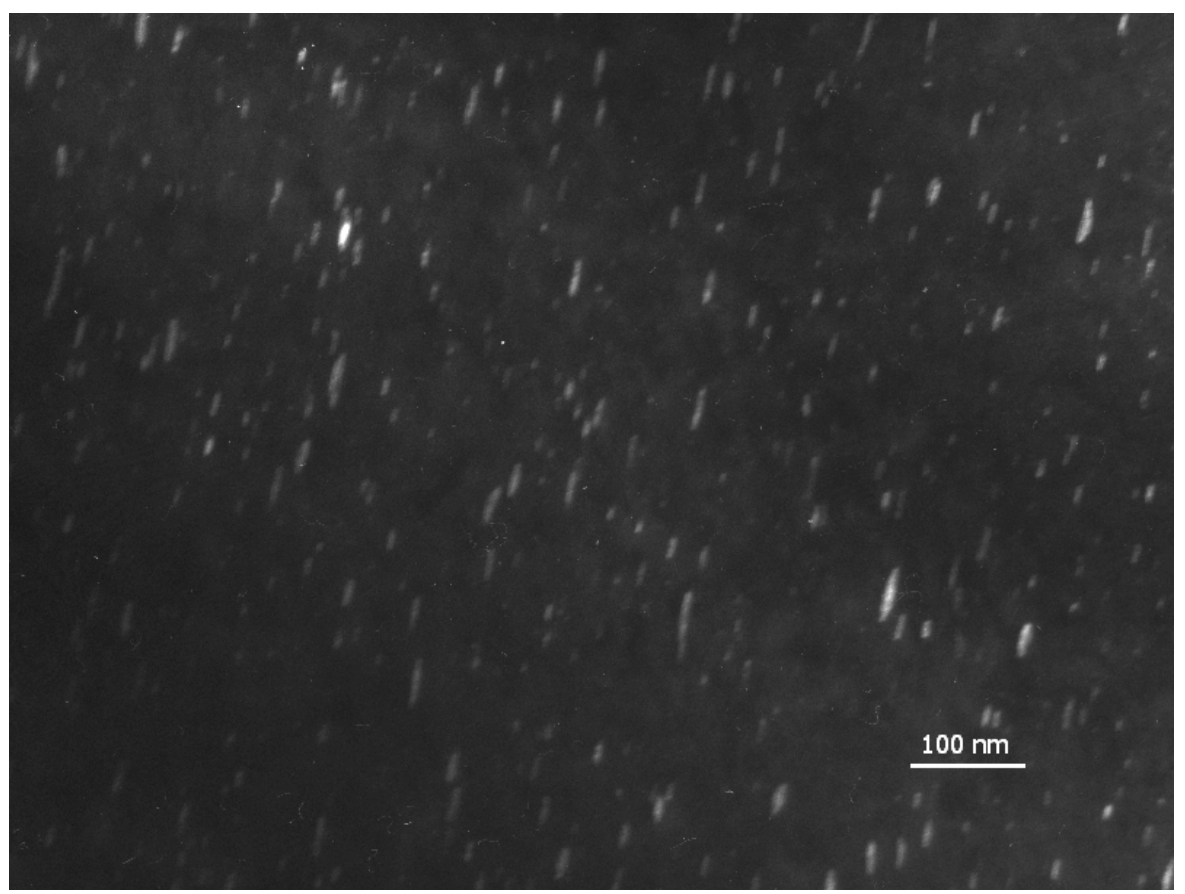

(c)

Figure 23. (Contd.)

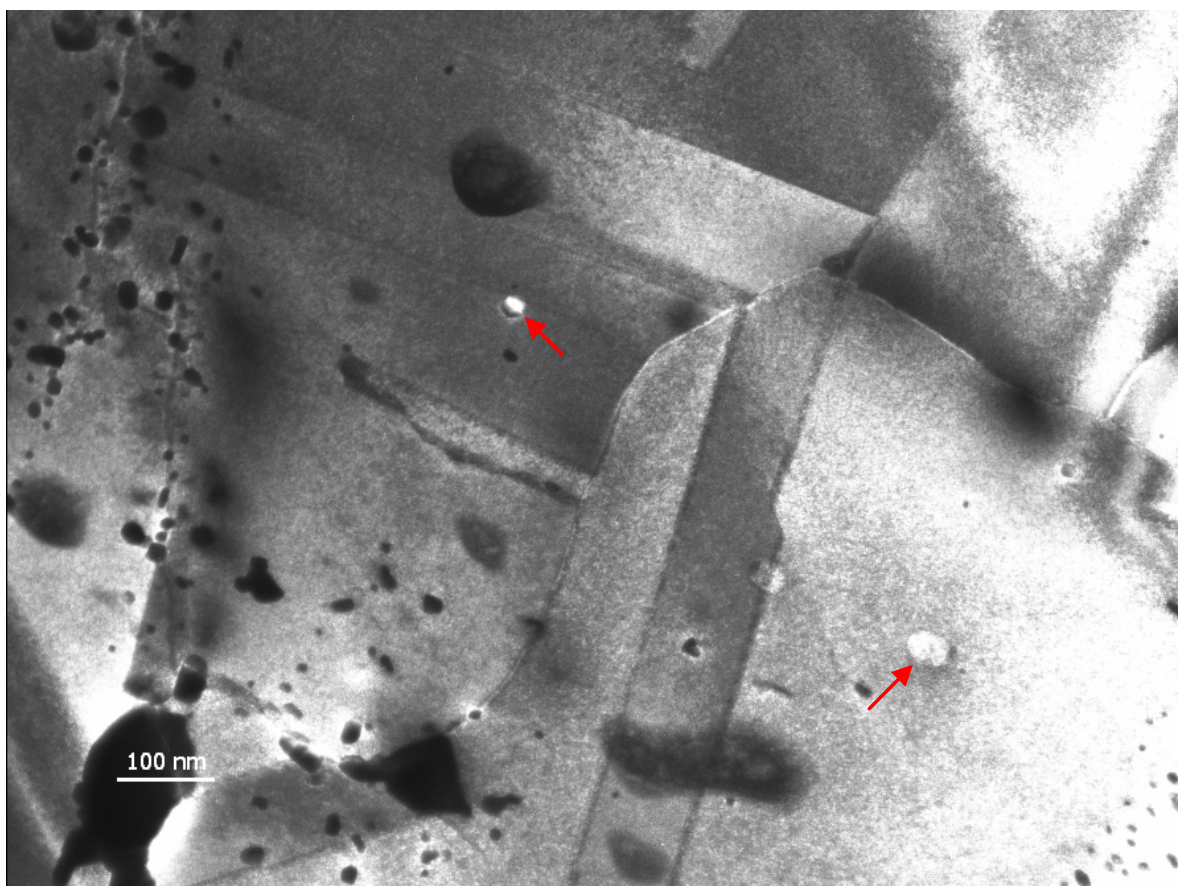

(a)

Figure 24. Irradiated microstructure of Alloy 690 (Mat. E2) at 24.5 dpa: (a) precipitates and cavities, (b) $\mathrm{BF}$ image of irradiation defects, and (c) relrod DF image of dislocation loops. 


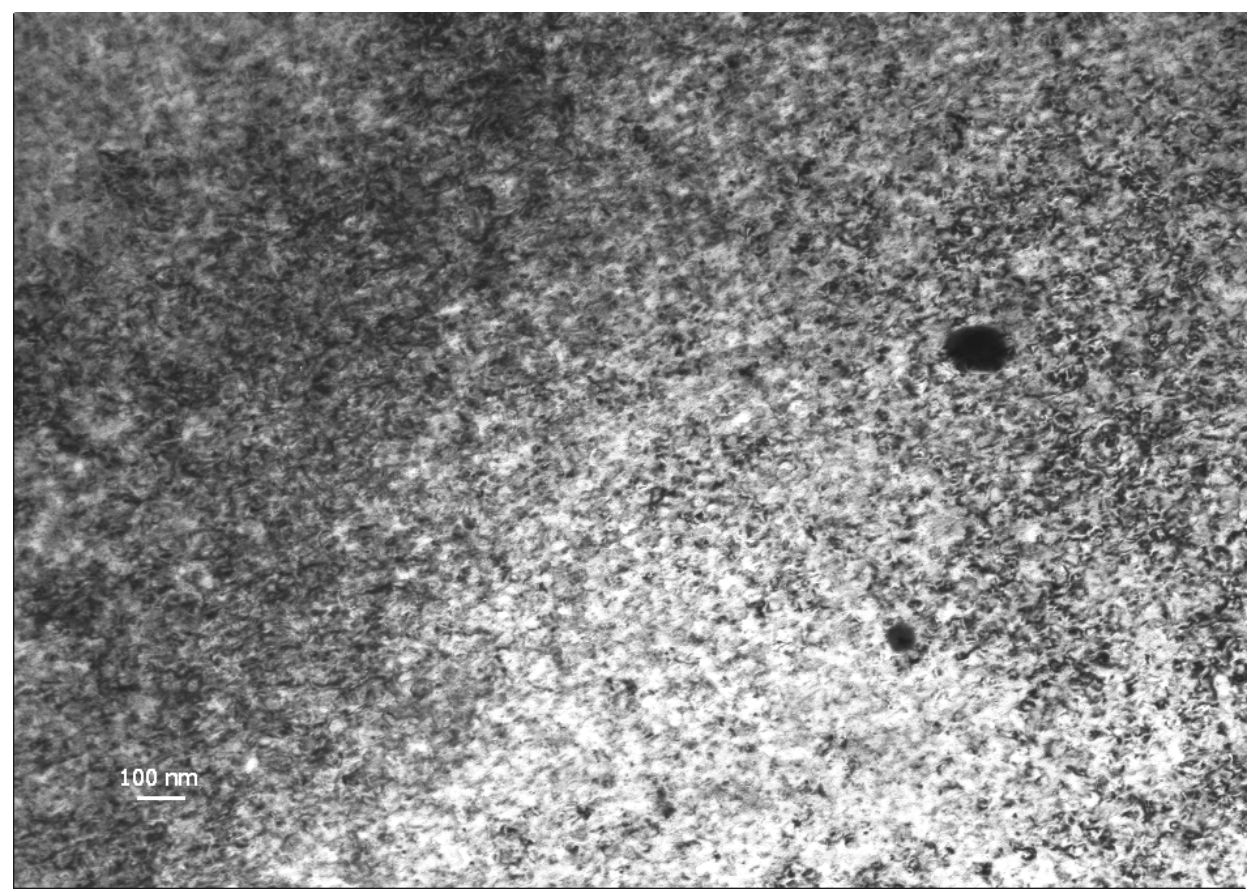

(b)



(c)

Figure 24. (Contd.) 




(a)

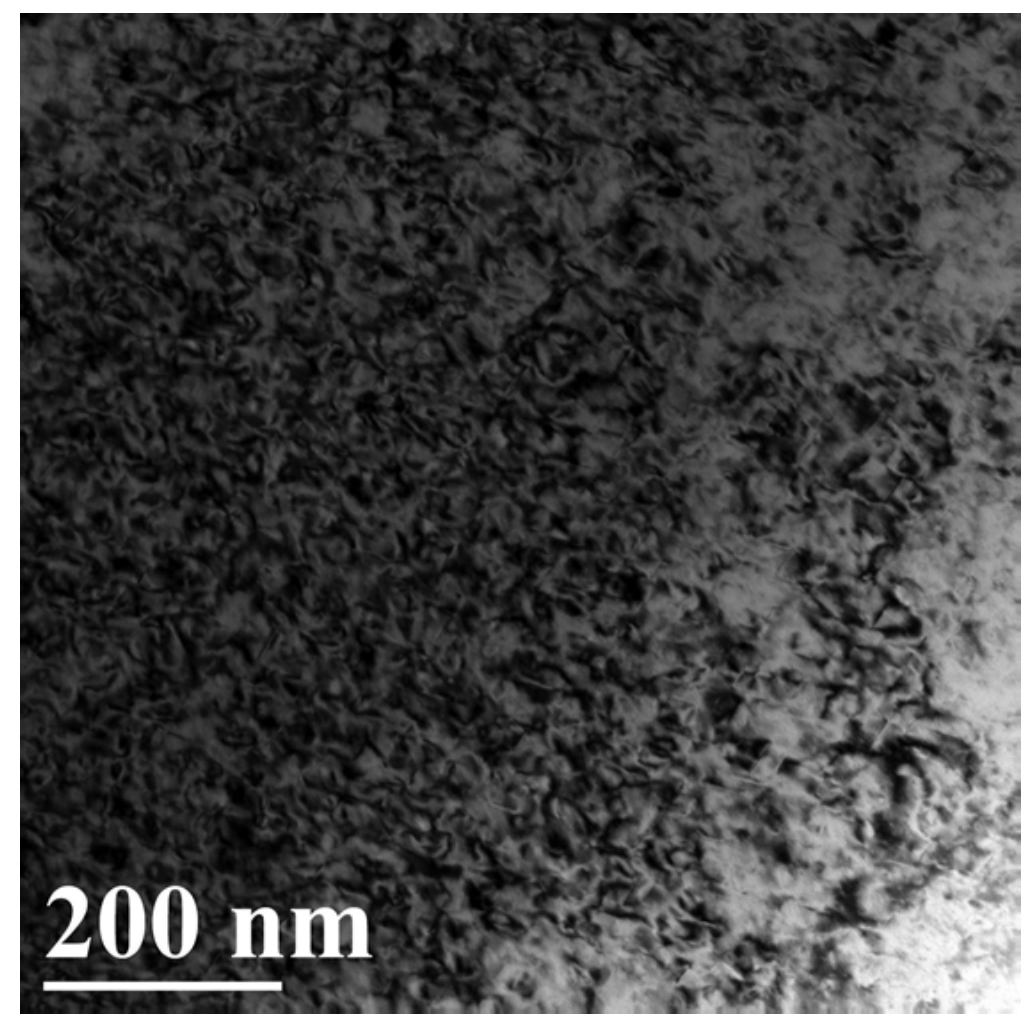

(b)

Figure 25. Irradiated microstructure of GBE 690 (Mat. E1) at 45 dpa. 



\section{Discussion}

A total of 26 TEM specimens irradiated in the BOR-60 reactor were examined for voids and irradiation defect structure in this study. The results are summarized in Table 12. No voids are observed in any sample, and the main irradiation defects are faulted dislocation loops in close-packed $\{111\}$ planes. Different types of austenitic SSs appear to have a similar irradiation-induced defect structure. The average loop size varies from 6 to $29 \mathrm{~nm}$, and the loop density is between 1 and $9 \times 10^{22} \mathrm{~m}^{-3}$. It has to be emphasized that a few larger loop size estimates may include measurement errors and overlapping loops. For Type 304 SSs and cast SSs, the size and density of dislocation loops are insensitive to small variations of sulfur or carbon contents. Pre-existing microstructure resulting from thermal-mechanical processing prior to irradiation seems to play an important role in defect evolution. Fine irradiation defects ("black dots") can be seen in the specimens with cold-work (B2) and inadequate solution-anneal treatments (B3 and B4). These irradiation defects are smaller than the faulted loops identified with the relrod technique in solution-annealed specimens.

Table 12. Summary of TEM observations on the irradiated BOR-60 specimens.

\begin{tabular}{|c|c|c|c|c|c|c|}
\hline Dose (dpa) & Material & Mat. Code & Void & $\begin{array}{l}\text { Irradiation-induced } \\
\text { Precipitates }\end{array}$ & $\begin{array}{c}\text { Average loop } \\
\text { size } \\
(\mathrm{nm}) \\
\end{array}$ & $\begin{array}{l}\text { Loop density } \\
\left(\mathrm{x} 10^{22} \mathrm{~m}^{-3}\right)\end{array}$ \\
\hline \multirow{2}{*}{5.5} & 304, high-S & $\mathrm{A} 2$ & Not observed & Not observed & 7.9 & 3.3 \\
\hline & $316 \mathrm{LN}$ & B3 & Not observed & Not observed & 8.7 & 3.5 \\
\hline \multirow{3}{*}{10.2} & 304, high-S & A2 & Not observed & Not observed & 8.2 & 4.2 \\
\hline & 304, low-S & A3 & Not observed & Not observed & 8.1 & 2.7 \\
\hline & $316 \mathrm{LN}$ & B3 & Not observed & Not observed & 8.8 & 5.8 \\
\hline \multirow{11}{*}{24.5} & 304, high-S & A2 & Not observed & Not observed & 8.1 & 4.6 \\
\hline & 304, low-S & A3 & Not observed & Not observed & 25.5 & 3.0 \\
\hline & $304 \mathrm{CW}$ & B2 & Not observed & Not observed & - & - \\
\hline & HP 304L & A8 & Not observed & Not observed & 12.2 & 1.2 \\
\hline & $347 \mathrm{SA}$ & D1 & Not observed & Not observed & 9.4 & 2.0 \\
\hline & $316 \mathrm{LN}$ & B3 & Not observed & Present & 5.8 & 1.7 \\
\hline & $\mathrm{CF}-3$ cast & $\mathrm{C} 1$ & Not observed & Not observed & 8.5 & 2.8 \\
\hline & $\mathrm{CF}-8$ cast & $\mathrm{C} 2$ & Not observed & Not observed & 7.7 & 3.1 \\
\hline & CF -8 cast & $\mathrm{C} 4$ & Not observed & Not observed & 16.3 & 1.7 \\
\hline & GBE690 & E1 & Not observed ${ }^{a}$ & $\mathrm{~N} / \mathrm{A}^{\mathrm{b}}$ & 17 & 1.4 \\
\hline & Alloy690 & E2 & Not observed ${ }^{a}$ & $\mathrm{~N} / \mathrm{A}^{\mathrm{b}}$ & 28.9 & 1.1 \\
\hline \multirow{10}{*}{45.0} & 304 , high-S & $\mathrm{A} 2$ & Not observed & Possible & 7.8 & 6.7 \\
\hline & 304, low-S & A3 & Not observed & Possible & 7.3 & 5.8 \\
\hline & $304 \mathrm{SA}$ & $\mathrm{B} 1$ & Not observed & Not observed & 10.5 & 5.7 \\
\hline & HP 304L & A8 & Not observed & Not observed & 12.1 & 5.3 \\
\hline & $347 \mathrm{SA}$ & D1 & Not observed & Present & 10.8 & 5.9 \\
\hline & $316 \mathrm{LN}$ & B3 & Not observed & Present & - & - \\
\hline & $316 \mathrm{LN}-\mathrm{Ti}$ & B4 & Not observed & Present & - & - \\
\hline & $\mathrm{CF}-3$ cast & $\mathrm{C} 1$ & Not observed & Present & 10.1 & 6.2 \\
\hline & CF-8 cast & $\mathrm{C} 2$ & Not observed & Present & 9.0 & 9.2 \\
\hline & GBE690 & $\mathrm{E} 1$ & Not observed $^{\text {a }}$ & $\mathrm{N} / \mathrm{A}^{\mathrm{b}}$ & - & - \\
\hline
\end{tabular}

a Cavities most likely resulting from fall-off of precipitates were observed.

$\mathrm{b}$ Precipitates were present both before and after irradiation; however, irradiation-induced precipitation was not analyzed.

\subsection{Void Swelling under PWR-Relevant Conditions}

In this study, no voids were observed in all specimens with the through-focus technique under high magnification. Due to the TEM resolution limit, voids less than $1 \mathrm{~nm}$ could not be resolved, and thus the presence of very fine voids cannot be ruled out. Despite this uncertainty, it is clear that no significant void swelling occurred in the examined alloys up to $45 \mathrm{dpa}$. This result is consistent with an earlier 
review performed by Chung ${ }^{18}$ and another observation on a different group of BOR-60 specimens analyzed by Renault et al. ${ }^{23}$ The absence of voids, even at the highest dose, suggests that the irradiation condition in this study was inadequate for developing a steady-state growth of voids. To understand this result and its implication for PWR internals, we must understand the mechanism of void swelling and the variables that have significant impacts on void formation.

\subsubsection{Void Swelling Mechanism and Key Variables}

Void swelling has been studied intensively since its first discovery in 1967.12 A general description behind the micro-mechanism of void swelling has been established after decades of research. ${ }^{5-7}$ Void formation is a result of irradiation microstructural evolution, and the interactions between point defects and dislocations provide the fundamental driving force of void nucleation and growth. It is well accepted that elastic interactions between self-interstitials and dislocations are stronger than those between vacancies and dislocations. ${ }^{24}$ This difference is due to a relatively larger strain field surrounding a selfinterstitial than a vacancy. A biased attraction between self-interstitials and dislocation loops leads to a preferential interstitial flux toward dislocations. ${ }^{25}$ Subsequently, excess vacancies become supersaturated, and void nucleation and growth take place in irradiated materials. A theoretical treatment based on the rate theory has been developed for void swelling. ${ }^{4,5,26}$

As an irradiation effect, void swelling is a function of neutron dose. The development of swelling can be generally divided into two stages, an initial incubation period with a negligible swelling rate and a steadystate growth period with a constant growth rate. ${ }^{7,8}$ A dose threshold for the incubation period is thought to be necessary to build up a high enough dislocation density to induce a net flow of vacancies toward voids. The incubation period is affected by a number of variables. Irradiation temperature, dose rate, initial microstructure, and transmutation products all have significant impacts on the incubation period. 7,8 However, once a steady state is reached, the growth rate is somewhat less sensitive to experimental variables, and a constant swelling rate of $1 \%$ /dpa is typical for many austenitic SSs. 7,8 Void swelling data measured for CW Type 316 SS hex cans in fuel subassemblies from Experimental Breeder Reactor-II (EBR-II) illustrate the transition from the incubation period to steady-state growth (Fig. 26). ${ }^{27}$ Note that densification due to precipitation or dislocation activities were also observed at low doses for the $\mathrm{CW}$ Type 316 SS.

Since only surviving point defects contribute to microstructural evolution in irradiated materials, both defect production and annihilation rates influence void formation. For this reason, void swelling is affected by the irradiation temperature and displacement damage rate. At low temperatures, vacancy mobility is low, and thus the recombination rate with mobile interstitials is high. As a result, few surviving vacancies contribute to void formation and growth. At high temperatures, voids become unstable due to thermal emission (i.e. vacancies dissociate from vacancy clusters and become thermal equilibrium vacancies). Thus, the recombination rate of vacancies and interstitials is also high. Void swelling peaks around an intermediate temperature. This characteristic peak temperature is usually around $0.3-0.5$ of the melting temperature $\left(T_{m}\right)$. Figure 27 shows a schematic of swelling behavior as a function of irradiation temperature and dose. 


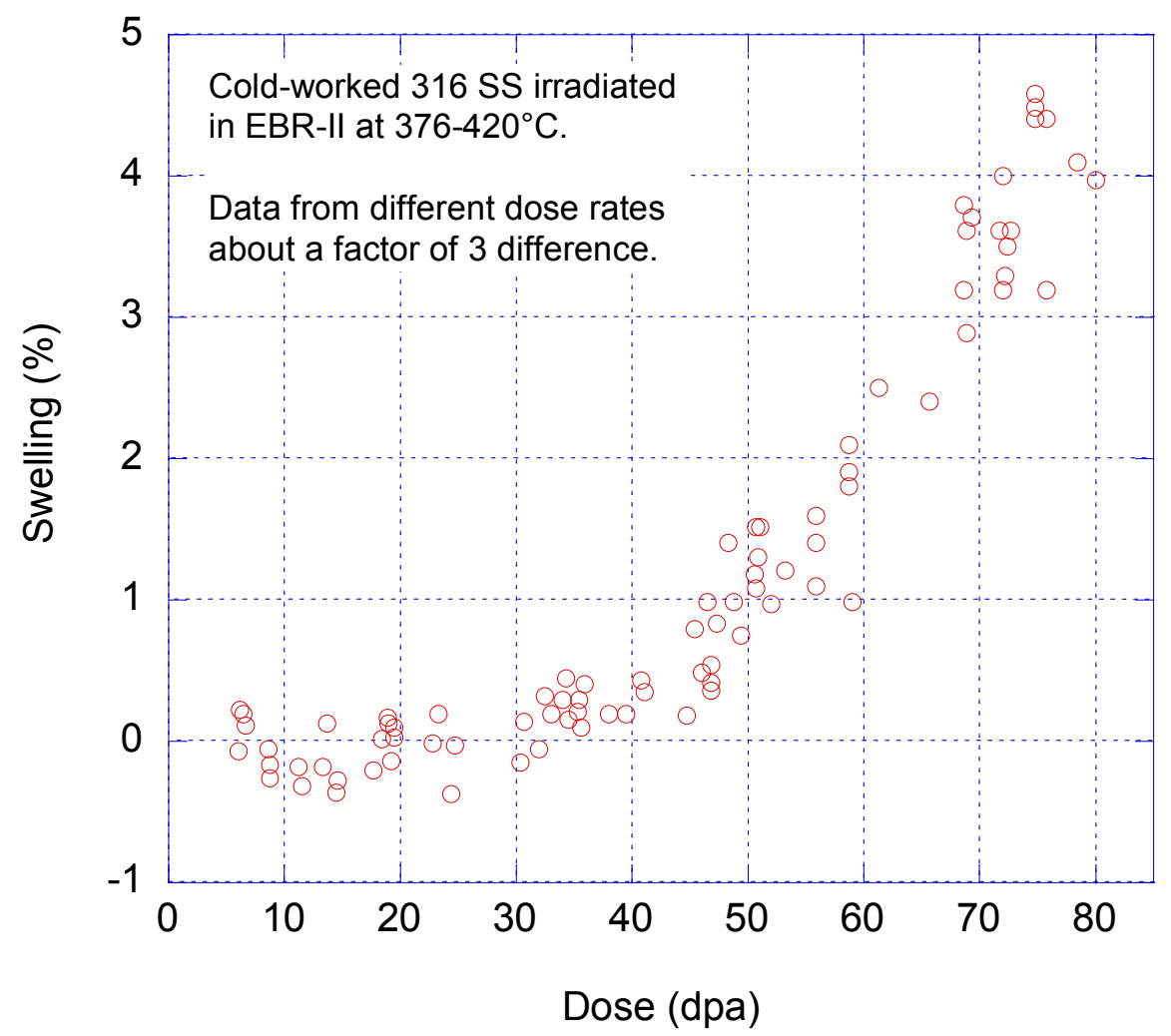

Figure 26. Void swelling measured on $12 \%$ CW 316 SSs irradiated in EBR-II. ${ }^{27}$

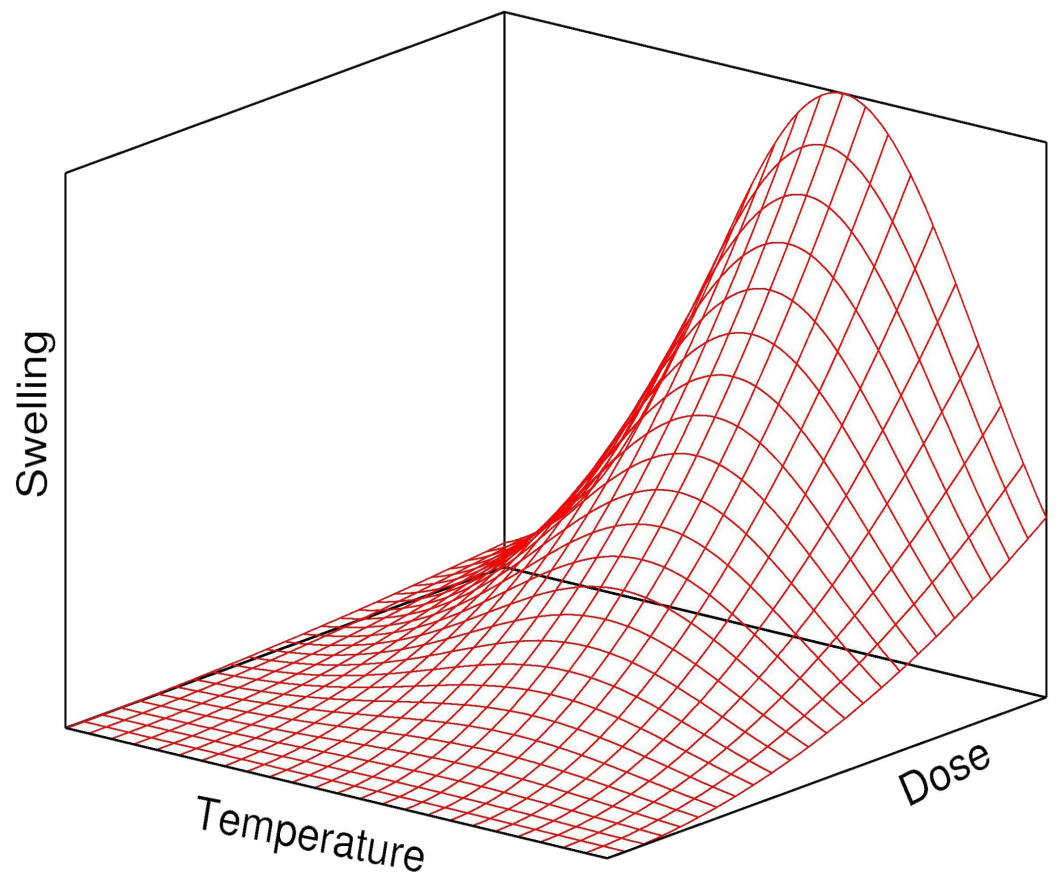

Figure 27. Schematic of temperature and dose dependence of void swelling. 5 
Besides the influence of temperature, void formation also depends on the production rate of point defects. Therefore, void swelling is a function of displacement damage rate or dose rate. Since both recombination rate and production rate impact the number of surviving point defects, the effects of temperature and dose rate are inherently coupled. Both experimental and theoretical studies have shown a clear correlation between temperature and dose rate on swelling development. ${ }^{28,29}$ A shift in peak swelling temperature to lower values is anticipated with increasing dose rates. The line in Fig. 28, adapted from an ion irradiation study of nickel, ${ }^{28}$ clearly shows that the dose rate and temperature are correlated. While the dose rates of ion irradiation may be too high for a PWR condition, this temperature shift is expected to extend into PWR-relevant damage rates. In a study by Allen et al. ${ }^{30}$, void swelling was found to increase with decreasing dose rate between 0.5 and $5.8 \times 10^{-7} \mathrm{dpa} / \mathrm{s}$ for SSs.

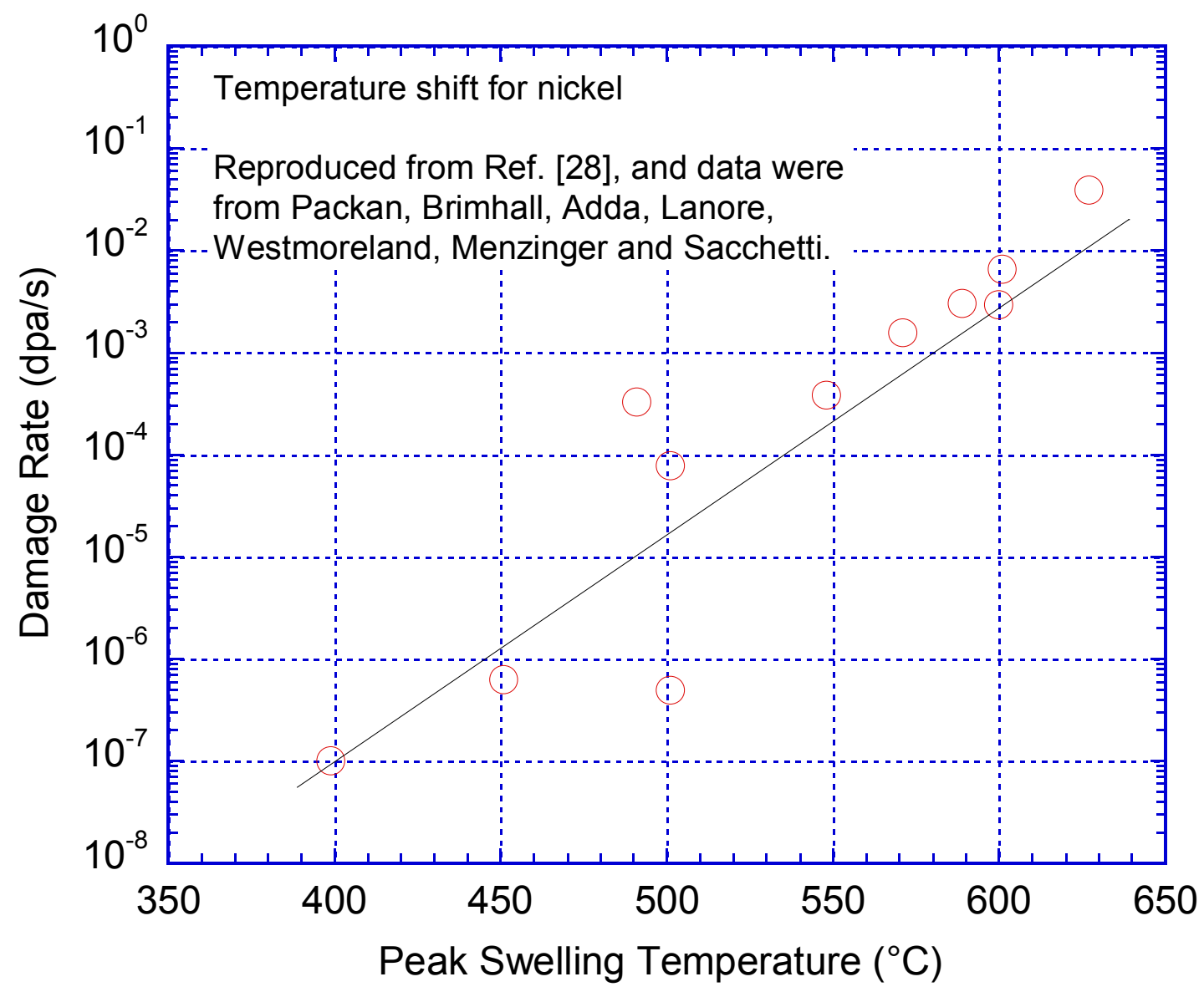

Figure 28. Relation of peak swelling temperature and dose rate. ${ }^{28}$

As shown in Fig. 29, cold-work has a significant effect on void swelling as well. Garner showed that cold-work effectively prolongs the incubation period of void swelling. ${ }^{7}$ Other studies have also shown that, at the same irradiation temperature, the onset of linear swelling rate can be delayed considerably by cold-work. Complex dislocation microstructure generated by cold-work is thought to affect the evolution of irradiation defects, and thus contribute to void swelling. The effect of cold-work is also limited to the incubation period, and there is little difference in the steady-state swelling rate between cold-worked and solution-annealed SSs. With the increase of irradiation dose, network dislocations will saturate and a steady state can be reached. Thus, a linear swelling rate (steady-state) will eventually prevail with increasing dose regardless of initial microstructure. 




Figure 29. Effect of cold-work on void swelling. ${ }^{7}$

After the first discovery of void swelling, it was verified that the micro-voids in irradiated SSs are not gas bubbles, which require sufficient pressure to maintain equilibrium at the bubble surface. ${ }^{12}$ However, transmutation gases, such as hydrogen and helium, are still critical for void development. Numerous experimental studies showed that gas atoms stabilize void nuclei and play a significant role in void development. ${ }^{37}$ Mansur showed that, from a theoretical standpoint, gas atoms must be present to stabilize vacancy clusters to reach a critical radius for growth. 5,26 This critical role is mainly fulfilled by helium, a transmutation product under neutron irradiation, in void formation. The most significant contributors to helium production are nickel and boron in austenitic SSs. For nickel, helium can come from two types of reactions, a threshold $(\mathrm{n}, \alpha)$ reaction or a two-step ${ }^{58} \mathrm{Ni}(\mathrm{n}, \gamma)^{59} \mathrm{Ni}(\mathrm{n}, \alpha)^{56} \mathrm{Fe}$ reaction. The two-step reaction operates strongly in a thermal spectrum, which becomes a dominant factor of helium production in SSs. ${ }^{31,32}$ The two-step reaction leads to a delay in helium production compared to the single-step reaction. Sufficient exposure to thermal neutrons is needed to build up the ${ }^{59} \mathrm{Ni}$ population. This behavior implies that a shift in helium production per dpa toward high dose levels.

In addition, void swelling can be affected by crystal structure, grain size, alloy elements, and applied stress. The steady-state swelling rate was found to be much lower for ferrite than austenite in steels. ${ }^{33}$ The low swelling rate of the body-centered cubic (bcc) structure is attributed to a more open crystal structure, and thus a higher self-diffusion rate. A mechanism that involves point defect trapping at solutes also contributes to the low swelling rate in the bcc structure. ${ }^{34}$ Furthermore, grain boundaries have a significant impact on void swelling. Singh and Foreman ${ }^{35}$ showed that, by acting as un-biased sinks, 
grain boundaries enhance point defect combinations and, therefore, affect void nucleation around grain boundaries. This effect leads to void-denuded zones around grain boundaries, which can be observed experimentally in different alloys. ${ }^{36}$ Alloy elements, such as Ni, Si, P, Ti and C have different effects on swelling behavior in different alloys, ${ }^{37}$ and the effects of alloy elements often vary at different temperatures. Since void swelling is a result of the partition of irradiation-induced point defects among different sinks, alloy elements in solutes or precipitates will certainly contribute differently to microstructural evolution. For this reason, the role of alloy elements can be sensitive to the thermal mechanical treatment. Finally, applied stress is also critical to swelling, ${ }^{38}$ as it can shorten the incubation period. Because of the effect of stress, void swelling is inherently related to irradiation creep, another phenomenon that also involves the flow of irradiation-induced point defects under stress.

\subsubsection{Irradiation Conditions of BOR-60 and PWR Internals}

As discussed above, void swelling in SSs is sensitive to a number of material and irradiation variables. To evaluate the results of the BOR-60 specimens and their implications with regard to the behavior of PWR internals, a careful analysis of irradiation conditions is needed. The focus in this discussion will be on the differences between the BOR-60 and typical PWR irradiations that could contribute significantly to void swelling.

\subsubsection{Irradiation temperature}

Irradiation temperature is perhaps the most important factor that affects the transient regime of void swelling. The BOR-60 irradiation was performed at $\sim 320^{\circ} \mathrm{C}$, a typical service temperature of PWR internals. Exposed to sodium coolant directly, the irradiation temperature of the BOR-60 specimens was largely controlled by the inlet sodium temperature, which is between $300-330^{\circ} \mathrm{C}$ for the BOR-60 reactor. $^{21}$ This inlet temperature is lower than most western-designed fast breeder reactors, but is adequate for simulating PWR-relevant irradiation temperatures. Eutectic-alloy temperature monitors were also used in the BOR-60 irradiation to track the peak temperature during irradiation. ${ }^{21}$ Postirradiation examination of these thermal monitors confirmed that the irradiation capsule temperatures were all below $343^{\circ} \mathrm{C}$, suggesting a well-controlled coolant flow in irradiation capsules throughout the experiment.

Gamma-heating is another variable that could affect irradiation temperature. Heat generated by irradiation must be removed efficiently to control the irradiation temperature. The $\gamma$-heating is reported to be $\sim 4.86 \mathrm{~W} / \mathrm{g}$ for the BOR-60 reactor. ${ }^{21}$ Despite this high value, temperature increase in the TEM disks should be minimal because of their small dimensions. With adequate coolant flow in irradiation capsules, the temperature of the TEM disks should be fairly close to the inlet temperature. Thus, the irradiation temperature of the BOR-60 specimens is comparable to that of PWR internals. However, the irradiation temperatures of some thick components in PWRs (e.g., re-entrant corners) could be significantly higher due to $\gamma$-heating. ${ }^{18}$ Since the incubation period of void swelling is sensitive to irradiation temperature, these thick sections are more vulnerable for void swelling in PWR internals. The sensitivity to temperature or $\gamma$-heating was demonstrated by a study on a CW 316 SS baffle bolt removed from the core of a PWR. ${ }^{39}$ In this work, TEM examinations were performed on bolt head, top shank, and threads. While no voids were present at the bolt head, they were observed at the top shank and threads. This observation was attributed to the temperature difference at these locations. While the bolt head was irradiated at the reactor coolant temperature, the shank and threads were subjected to higher temperatures due to $\gamma$ - heating. 


\subsubsection{Dose rate}

As discussed earlier, the effects of irradiation temperature and dose rate are coupled. The damage rate of the BOR-60 irradiation is about $10^{-6} \mathrm{dpa} / \mathrm{s}$, at least one order of magnitude higher than that of PWRs. This high dose rate suggests an upward shift of $40-60^{\circ} \mathrm{C}$ of the peak swelling temperature. ${ }^{7}$ If this "temperature shift" is indeed adequate at the PWR dose rates (less than $10^{-7} \mathrm{dpa} / \mathrm{s}$ ), irradiation temperatures of $360-380^{\circ} \mathrm{C}$ at the BOR- 60 damage rate would be more appropriate for simulating a PWR irradiation at $320^{\circ} \mathrm{C}$. This estimate, of course, is based on an assumption that other thermal effects, such as precipitation or solute-trapping, remain the same within the range of the temperature shift. Limited data have shown that voids can develop at PWR-relevant dose rates and rather low temperatures. ${ }^{16,17,39-}$

41 More important, these studies showed that, if dose rates are sufficiently low, voids can be found at surprisingly low doses.

\subsubsection{Neutron spectrum}

As a fast breeder reactor, the neutron spectrum of the BOR-60 is different from that of PWRs. Without the water moderator, the neutron spectrum of a fast reactor is harder (the population of fast neutrons is higher) than that of an LWR. While fast neutrons are more efficient in generating displacement damage, thermal neutrons contribute more to transmutations, which produce gas atoms such as helium or hydrogen. ${ }^{42}$ Thus, the ratio of gas production and displacement damage (termed "gas production rate" with units of appm/dpa) is significantly higher in a PWR than in the BOR-60 reactor. As discussed earlier, gas atoms, especially helium, stabilize void nuclei and thus facilitate void formation. ${ }^{32}$ It is possible that the difference in swelling behavior between fast reactor and PWR irradiations (compared at the same dpa level) is caused by the difference in helium production rate. At the same irradiation temperature, both the low gas production rate (appm/dpa) and the high damage rate (dpa/s) in the BOR-60 irradiation could hinder the void formation, leading to a longer incubation period of void swelling. This may be the reason that voids are not observed among the specimens examined in the current study. Additional research is needed to fully understand the effect of the neutron spectrum on void swelling.

\subsection{Irradiated Microstructure at PWR-Relevant Temperature}

For the specimens examined in this study, the irradiated microstructures are dominated by a high density of dislocation loops. The mean size of these dislocation loops varies from $\sim 6 \mathrm{~nm}$ in Type $316 \mathrm{LN}$ SS to $\sim 25 \mathrm{~nm}$ in low-S Type $304 \mathrm{SS}$. For a given material, the mean size of the dislocation loops seems not to change considerably with neutron dose from 5.5 to $45 \mathrm{dpa}$. The density of the dislocation loops, however, increases slightly from 5.5 to $10.2 \mathrm{dpa}$, but saturates above $10 \mathrm{dpa}$ at approximately $5 \times 10^{22} \mathrm{~m}^{-3}$. An example of the dose-dependent behavior of dislocation loops is shown in Fig. 30 from this study. These observations on dislocation loop size and density are consistent with literature values obtained from PWR internal components. In a study on a removed PWR baffle bolt between 7 and 19.5 dpa, Edwards et al. ${ }^{39}$ reported an average loop size of 7-12 nm and a loop density of $0.85-1.2 \times 10^{23} \mathrm{~m}^{-3}$ for CW $316 \mathrm{SS}$. In another study on a CW 316 SS flux thimble tube (33-70 dpa) removed from an European PWR, ${ }^{43}$ the loop size and density were found to be $8.5-10 \mathrm{~nm}$ and $0.76-1.5 \times 10^{23} \mathrm{~m}^{-3}$, respectively. A similar study was also performed by Fujii et al. on a flux thimble tube ( $35-53 \mathrm{dpa}$ ) from a Japanese PWR. ${ }^{40}$ A loop size of 10-15 nm and a density of $4-9 \times 10^{22} \mathrm{~m}^{-3}$ were reported. These results from real PWR internal components are very similar to the measurements obtained in the current study, suggesting a similar dislocation loop microstructure at PWR-relevant temperature. 


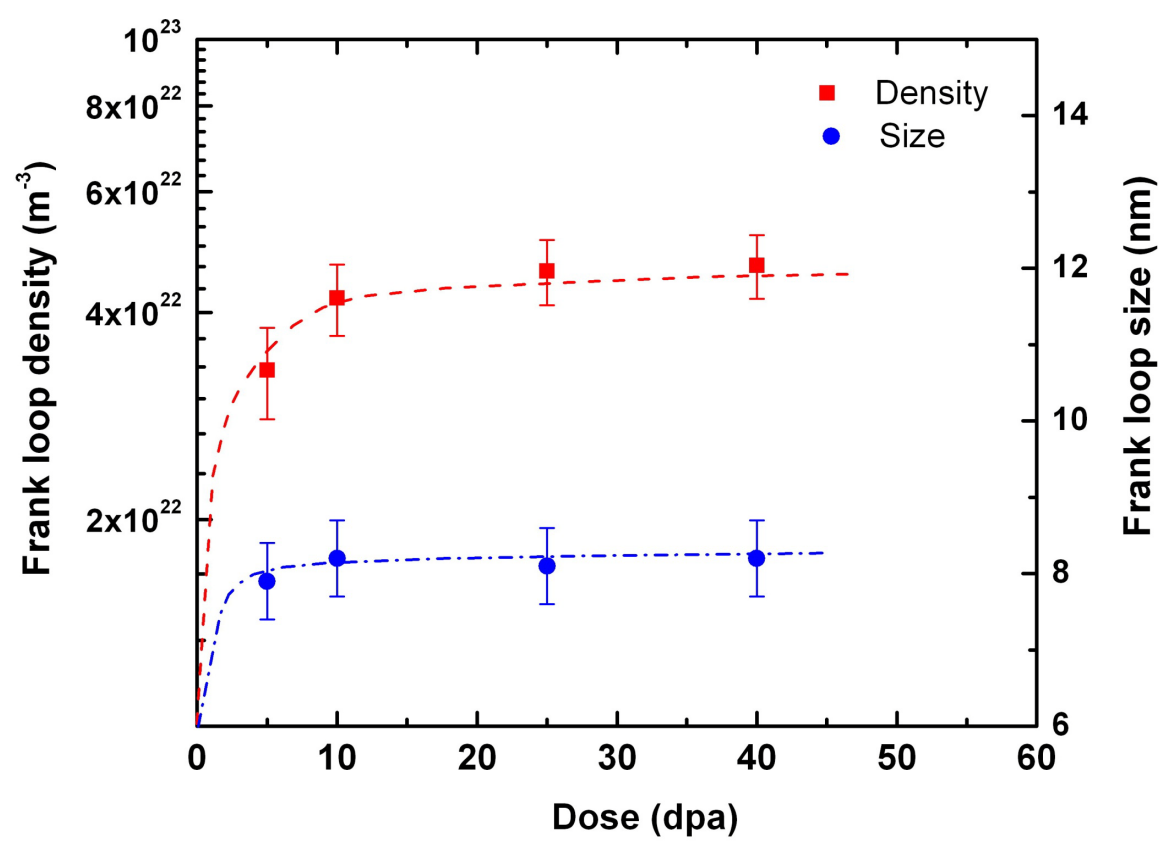

Figure 30. Density and size of Frank loops in irradiated 304 SA with high S content.

Among the 26 specimens in this study, no irradiation-induced precipitate was present below 24.5 dpa. Fine precipitates were observed in some 45-dpa specimens. Limited by the resolution of TEM, the exact nature of these fine precipitates cannot be determined. The irradiation-induced precipitates are more evident in cast SSs compared to wrought SSs. At $45 \mathrm{dpa}$, the average size and density of precipitates are $34 \mathrm{~nm}$ and $7.2 \times 10^{19} \mathrm{~m}^{-3}$, respectively, for CF-3 SS, and $6.7 \mathrm{~nm}$ and $7 \times 10^{21} \mathrm{~m}^{-3}$, respectively, for CF-8 SS. A much higher density of finer precipitates in the CF-8 grade SS may be attributed to its high carbon content. Precipitates were also observed in GBE Alloy 690 both before and after irradiation. The precipitates were identified as chromium-rich carbides with face-centered cubic structure. It appears that irradiation did not alter the pre-existing precipitates in the nickel alloy at this dose level. 


\section{Summary}

TEM microstructural examinations have been carried out on selected austenitic SSs, cast SSs and a nickel alloy that had been irradiated in the BOR- 60 reactor from 5.5 to $45 \mathrm{dpa}$ and $\sim 320^{\circ} \mathrm{C}$. The irradiated microstructures were dominated by a high density of dislocation loops, which varied in mean size and density for different alloys. While no irradiation-induced precipitates were present below $24.5 \mathrm{dpa}$, fine precipitates were evident in some 45-dpa specimens. The exact nature of these precipitates is unclear. No voids were observed in any specimens imaged with the through-focus technique under high magnification. Despite the possibility that fine voids below the TEM resolution limit may be present, it is clear that void swelling is insignificant in all examined alloys up to $45 \mathrm{dpa}$. The absence of voids, even at the highest dose, suggests that the irradiation conditions in this study may be inadequate to foster steadystate void growth, and void swelling is still within the incubation period. While the temperature of the BOR-60 irradiation $\left(\sim 320^{\circ} \mathrm{C}\right)$ is prototypical for PWR core internals, the displacement damage rate $\left(\sim 10^{-} 6\right.$ $\mathrm{dpa} / \mathrm{s})$ is higher than that typically experienced by PWR internals. A shift in peak swelling temperature may result from the high dose rate used in this study. In addition, a low population of thermal neutrons in the BOR-60 spectrum, which leads to a lower production rate of transmutation helium, may also contribute to the absence of void swelling. 



\section{References}

1. Robinson, M. T., "Basic Physics of Radiation Damage Production,” J. Nucl. Mater., 216 (1994) 128.

2. Greenwood, L. R., "Neutron Interactions and Atomic Recoil Spectra," J. Nucl. Mater., 216 (1994) $29-44$.

3. Averback, R. S., “Atomic Displacement Processes in Irradiated Metals,” J. Nucl. Mater., 216 (1994) 49-62.

4. Gittus, John, Irradiation Effects in Crystalline Solids, Applied Science Publishers, London, 1978.

5. Mansur, L. K., "Theory and Experimental Background on Dimensional Changes in Irradiated Alloys,” J. Nucl. Mater., 216 (1994) 97-123.

6. Mansur, L. K., E. H. Lee, P. J. Maziasz, and A. P. Rowcliffe, "Control of Helium Effects in Irradiated Materials Based on Theory and Experiment," J. Nucl. Mater., 141-143 (1983) 633-646

7. Garner, F. A., "Irradiation Performance of Cladding and Structural Steels in Liquid Metal Reactors," in Materials Science and Technology - A Comprehensive Treatment, R. W. Cahn, P. Haasen, and E. J. Kramer, eds., VCH Publishers Inc., New York, 1994.

8. Garner, F. A., "Recent Insights on the Swelling and Creep of Irradiated Austenitic Alloys," J. Nucl. Mater., 122\&123 (1984) 459-471.

9. Zinkle, S. J., P. J. Maziasz and R. E. Stoller, "Dose Dependence of the Microstructural Evolution in Neutron-Irradiated Austenitic Stainless Steel,”J. Nucl. Mater., 206 (1993) 266-286.

10. Maziasz, P. J., "Overview of Microstructural Evolution in Neutron-Irradiated Austenitic Stainless Steels”, J. Nucl. Mater., 205 (1993) 118-145.

11. Lucas, G. E., "Evolution of Mechanical Property Change in Irradiated Austenitic Stainless Steels," J. Nucl. Mater., 206 (1993) 287-305.

12. Cawthorne, C., and E. J. Fulton, "Void in Irradiated Stainless Steel," Nature, 216 (1967) 575.

13. Straalsund, J. L., R. W. Powell, and B. A. Chin, "An Overview of Neutron Irradiation Effects in LMFBR Materials,” J. Nucl. Mater., 108\&109 (1982) 299-305.

14. Harkness, S. D., and C. Y. Li, "Study of Void Formation in Fast Neutron-Irradiated Metals," Met. Trans., 2 (1971) 1457-1470.

15. Garner, F. A., L. R. Greenwood, and D. L. Harrod, "Potential High Fluence Response of Pressure Vessel Internals Constructed from Austenitic Stainless Steels,” Proc. 6th Intl. Symp. on Environmental Degradation of Materials in Nuclear Power Systems - Water Reactors, TMS, Warrendale, PA, 1993.

16. Garner, F. A., and M. B. Toloczko, "Irradiation Creep and Void Swelling of Austenitic Stainless Steels at Low Displacement Rates in Light Water Energy Systems," J. Nucl. Mater. 251 (1997) 252-261.

17. Porollo, S. I., Yu. V. Konobeev, A. M. Dvoriashin, V. M. Krigan, and F. A. Garner, "Determination of the Low Temperature Limit of Void Swelling of Stainless Steels at PWR-Relevant Displacement Rates," Proc. 10th Intl. Symp. on Environmental Degradation of Materials in Nuclear Power Systems - Water Reactors, NACE, Houston, TX, 2002.

18. Chung, H. M., "Assessment of Void Swelling in Austenitic Stainless Steel Core Internals," NUREG/CR-6897, ANL-04/28, Jan. 2006. 
19. Garner, F. A., L. R. Greenwood and D. L. Harrod, "Potential High Fluence Response of Pressure Vessel Internals Constructed from Austenitic Steels," Proc. 6th Intl. Symp. on Environmental Degradation of Materials in Nuclear Power Systems - Water Reactors, TMS, Warrendale, PA, 1993.

20. Was, G. S., and S. M. Bruemmer, "Effects of Irradiation on Intergranular Stress Corrosion Cracking," J. Nucl. Mater., 216 (1993) 326.

21. Electric Power Research Institute, "CIR II Program: Description of the Boris 6 and 7 Experiments in the BOR-60 Fast Breeder Reactor,” Report No. 1011787, Palo Alto, CA, 2005.

22. Roberts, A. C., and H. E. Evans, "Effects of Titanium and Silicon Additions on the Creep Behavior of TiN- Dispersion Strengthened 20Cr-25Ni Stainless Steel," Proc. Conf. Mech. Behavior and Nuclear Applications of Stainless Steel at Elevated Temperatures, London, Metals Society. Book No. 280, P51.

23. Renault, A. E., C. Pokor, J. Garnier, and J. Malaplate, "Microstructure and Grain Boundary Chemistry Evolution in Austenitic Stainless Steels Irradiated in the BOR-60 Reactor up to 120 dpa," 14th Int. Conf. on Environmental Degradation of Materials in Nuclear Power Systems, Virginia Beach, VA, August 23-27, 2009.

24. Hirth, J. P. and J. Lothe, Theory of Dislocations, $2^{\text {nd }}$ Ed., Krieger Publishing Company, Malabar, Florida, 1992.

25. Heald, P. T., "Preferential Trapping of Interstitials at Dislocations," Phil. Mag., 31, 3 (1975) 551.

26. Mansur, L. K., "Theory of Transitions in Dose Dependence of Radiation Effects in Structural Alloys," J. Nucl. Mater., 206 (1993) 306.

27. Allen, T. R., H. Tsai, R. S. Daum, D. L. Porter, J. I. Cole, T. Yoshitake, N. Akasaka, T. Donomae, S. Mizuta, J. Ohta, K. Dohi, and H. Kusanagi, "Effects of Irradiation on the Swelling and Mechanical Properties of 316 Stainless Steel," 11th Int. Conf. on Environmental Degradation of Materials in Nuclear Power Systems, Stevenson, WA, August 10-14, 2003.

28. Packan, N. H., K. Farrell, and J. O. Stiegler, "Correlation of Neutron and Heavy-Ion Damage, I. The Influence of Dose Rate and Injected Helium on Swelling in Pure Nickel," J. Nucl. Mater., 78 (1978) 143.

29. Mansur, L. K., "Correlation of Neutron and Heavy-Ion Damage, II. The Predicted Temperature Shift of Swelling with Changes in Radiation Dose Rate," J. Nucl. Mater., 78 (1978) 156.

30. Allen, T. R, J. I. Cole, C. L. Trybus, D. L. Porter, H. Tsai, F. Garner, E. A. Kenik, T. Yoshitake, and Joji Ohta, "The Effect of Dose Rate on the Response of Austenitic Stainless Steels to Neutron Radiation,” J. Nucl. Mater., 348 (2006) 148.

31. Greenwood, L. R, "A New Calculation of Thermal Neutron Damage and Helium Production in Nickel,” J. Nucl. Mater., 115 (1983) 137.

32. Greenwood, L. R., D. W. Kneff and R. P Skowronski, "A Comparison of Measured and Calculated Helium Production in Nickel using Newly Evaluated Neutron Cross Section for ${ }^{59} \mathrm{Ni}$," J. Nucl. Mater., 122 \&123 (1984) 1002.

33. Garner, F. A., and D. S. Gelles, "Neutron-Induced Swelling of Commercial Alloys at Very High Exposures," in Effects of Radiation on Materials, ASTM STP 1046, 1990.

34. Little, E. A., "Microstructure Evolution in Irradiated Ferritic-Martensitic Steels: Transitions to High Dose Behavior," J. Nucl. Mater., 206 (1993) 324. 
35. Singh, B. N., and A. J. E. Foreman, "Calculated Grain Size Dependent Vacancy Supersaturation and its Effect on Void Formation," Phil. Mag., 29 (1974) 847-57.

36. Singh, B. N., "On the Influence of Grain Boundaries on Void Growth," Phil. Mag., 28 (1973) 1409.

37. Lee, E. H., and L. K. Mansur, "Evidence for a Mechanism of Swelling Variation with Composition in Irradiated Fe-Cr-Ni Alloys," Philos. Mag. A52 (1985) 493.

38. Gelles, D. S., "Effects of Stress on Microstructural Evolution during Irradiation," J. Nucl. Mater., 205 (1993) 146.

39. Edwards, D. J., E. P. Simonen, F. A. Garner, L. R. Greenwood, B. M. Oliver, S. M. Bruemmer, "Influence of Irradiation Temperature and Dose Gradients on the Microstructural Evolution in Neutron-Irradiated 316SS," J. Nucl. Mater., 317 (2003) 32.

40. Fujii, K., K. Fukuya, G. Furutani, T. Torimaru, A. Kohyama and Y. Katoh, "Swelling in 316 Stainless Steel Irradiated to 53 dpa in a PWR," Proc. $10^{\text {th }}$ Intl. Conf. on Environmental Degradation of Materials in Nuclear Power Systems-Water Reactors, NACE/ANS/TMS, August 5-9, 2001.

41. Foster, J. P., D. L. Porter, D. L. Harrod, T. R. Mager, and M. G. Burke, "316 Stainless Steel Cavity Swelling in a PWR,” J. Nucl. Mater., 224 (1995) 207.

42. Garner, F. A., B. M. Oliver, L. R. Greenwood, D. J. Edwards and S. M. Bruemmer, "Generation and Retention of Helium and Hydrogen in Austenitic Steels Irradiated in a Variety of LWR and Test Reactor Spectral Environments," Proc. 10th Intl. Conf. on Environmental Degradation of Materials in Nuclear Power Systems-Water Reactors, NACE/ANS/TMS, August 5-9, 2001.

43. Edwards, D. J., F. A. Garner, S. M. Bruemmer, P. Efsing, "Nano-cavities Observed in a 316SS PWR Flux Thimble Tube Irradiated to 33 and 70 dpa," J. Nucl. Mater., 384 (2009) 249. 



\section{BIBLIOGRAPHIC DATA SHEET}

(See instructions on the reverse)
(Assigned by NRC, Add Vol., Supp., Rev.,

and Addendum Numbers, if any.)

NUREG/CR-7128

ANL-11/27
2. TITLE AND SUBTITLE

Void Swelling and Microstructure of Austenitic Stainless Steels Irradiated in the BOR-60 Reactor
3. DATE REPORT PUBLISHED MONTH TEAR

November 2012

4. FIN OR GRANT NUMBER

N-6519

5. AUTHOR(S)

Y. Chen 1, Y. Yang,2 Y. Huang2, T. Allen2, B. Alexandreanu1, and K. Natesan1

6. TYPE OF REPORT

Technical

7. PERIOD COVERED (Inclusive Dates)

8. PERFORMING ORGANIZATION - NAME AND ADDRESS (If NRC, provide Division, Office or Region, U. S. Nuclear Regulatory Commission, and mailing address; if contractor, provide name and mailing address.)

1Argonne National Laboratory, Argonne, IL 60439

2 University of Wisconsin-Madison, Madison, WI 53706

. SPONSORING ORGANIZATION - NAME AND ADDRESS (If NRC, type "Same as above", if contractor, provide NRC Division, Office or Region, U. S. Nuclear Regulatory Commission, and mailing address.)

Division of Engineering, Office of Nuclear Regulatory Research, U. S. Nuclear Regulatory Commission, Washington,DC 20555-0001

10. SUPPLEMENTARY NOTES

Appajosula S. Rao, Program Manager

11. ABSTRACT (200 words or less)

As nuclear power plants age and neutron fluence increases, detrimental effects resulting from radiation damage have become an increasingly important issue for the operational safety and structural integrity of core internal components. In this study, irradiated specimens of reactor core internal components were characterized by transmission electron microscopy. The specimens had been irradiated to $5.5-45 \mathrm{dpa}$ in the BOR-60 reactor at a dose rate close to $10-6 \mathrm{dpa} / \mathrm{s}$ and temperature of about $320^{\circ} \mathrm{C}$. No voids were observed in the austenitic stainless steels and nickel alloys at all doses. Despite the possibility that fine voids below the TEM resolution limit may be present, it was clear that void swelling was insignificant in all examined alloys up to 45 dpa. Irradiated microstructures of the studied alloys were dominated by a high density of Frank loops. The mean size and density of the Frank loops varied from one material to another, but saturated with increasing dose above $\sim 10 \mathrm{dpa}$. While no irradiation-induced precipitations were present below $24.5 \mathrm{dpa}$, fine precipitates were evident in several alloys at $45 \mathrm{dpa}$.

12. KEY WORDS/DESCRIPTORS (List words or phrases that will assist researchers in locating the report.)

BOR-60 Irradiation, Austenitic Stainless Steel, Microstructure, Dislocations and Dislocation Loops, irradiation induced precipitation



NRC FORM $335(12-2010)$ 




Printed
on recycled
paper

paper

Federal Recycling Program 



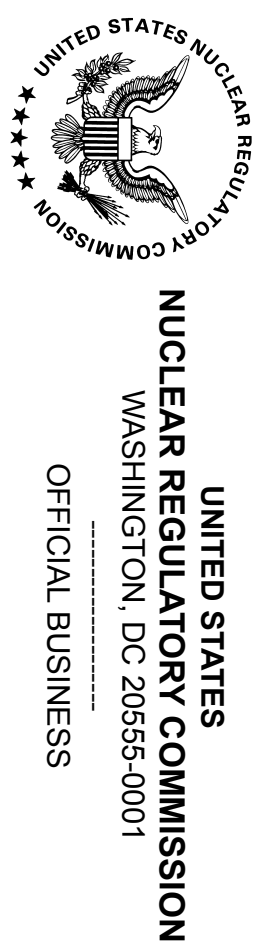



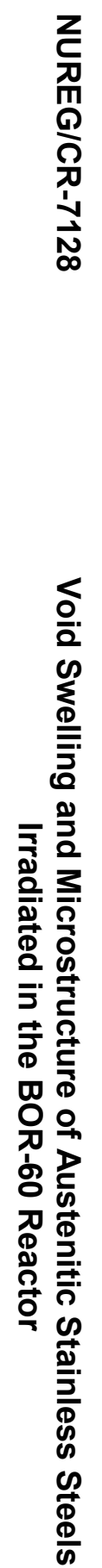

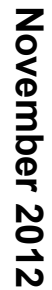

